\title{
Anatomical Optical Coherence Tomography in the Human Upper Airway
}

\author{
Julian J. Armstrong
}

This thesis is presented for the degree of

Doctor of Philosophy

of The University of Western Australia

School of Electrical, Electronic \& Computer Engineering.

April 2006 


\begin{abstract}
This thesis describes the development, clinical validation and initial application of a technique for taking measurements of the shape and dimensions of the human upper airway, called anatomical optical coherence tomography (aOCT). The technique uses a transparent catheter containing a rotating optical probe which is introduced transnasally and positioned in the airway and oesophagus. Optical coherence tomography is used to take calibrated cross-sectional images of the airway lumen as the probe rotates. The probe can also be advanced or withdrawn within the catheter during scanning to build up three-dimensional information. The catheter remains stationary so that the subject is not aware of the probe motion.

The initial application of the system is research into obstructive sleep apnoea (OSA), a serious condition characterized by repetitive collapse of the upper airway during sleep and an independent risk factor for deaths by heart disease, strokes or car accidents. Measurement of upper airway size and shape is important for the investigation of the pathophysiology of OSA, and for the development and assesment of new treatments. Obtaining such measurements during sleep is crucial, as a number of factors such as muscle tone, neural activity and instinctive respiration triggers are different during sleep. Current technologies such as CT, MRI, fluoroscopy, acoustic reflection and ultrasound are not able to routinely acquire such measurements of upper airway for extended periods of time or during fully characterized sleep. Using the aOCT system we have taken these measurements for the first time, and as the system is portable it can be used in normal hospital rooms. We have used $a$ OCT to capture three-dimensional data sets of the airway shape from upper oesophagus to the nasal cavity, undertaken measurements of compliance and other airway characteristics, and recorded dynamic airway shape during confirmed sleep apnoea events in a hospital sleep laboratory.

We have shown that $a \mathrm{OCT}$ generates quantitative, real-time measurements of upper airway size and shape, allowing study over lengthy periods during both sleep and wakefulness. These features should make it useful for study of upper airway behavior to investigate OSA pathophysiology, and aid clinical management and treatment development.
\end{abstract}




\section{Acknowledgements}

I would firstly like to thank David Sampson for being a great PhD supervisor, for offering the project to me, providing help and guidance throughout the project, and his work to ensure the availability of the resources and environment in OBEL necessary for it to be successful. He also facilitated other opportunities such as international collaboration and conference trips.

It has been a pleasure to work with our collaborators at the West Australian Sleep Disorders Research Institute at Sir Charles Gairdner Hospital. Thank you to Peter Eastwood and David Hillman for their many explanations to help me understand airway anatomy and sleep apnoea, their guidance and help in carrying out the medical experiments and writing medical research papers, helping with the interpretation of the data, and their patience and encouragement throughout the project. Peter particularly deserves thanks for being the first and continually enthusiastic volunteer to be scanned by the system and his work in organizing things over at the hospital. I gratefully thank Jenny Walsh, Kathy Maddison, Kelly Shepherd and Danni Philippe for their help with experiments and data analysis, and for volunteering for the validation experiments.

I would like to thank everyone who has worked with me on the system. Particularly, I've worked closely with Matthew Leigh which has been great. Also other OBEL people along the way - Stefan Schwer, Ian Walton, Andrei Zvyagin, Sergey Alexandrov, Alex Paduch. Mark Dowley and Rainer Rawer did some initial work on this project before I started the thesis. Thank you to Dilusha Silva for his work on the log-amp circuit and delay line, Steve Frisken for helping with the optical probe and the Department workshops for their expertise.

Thanks go to the OBEL postgrad students for their help, numerous interesting and stimulating discussions, and friendship - Dilusha, Kirrily, Simon, Bruce, Matthew, Timothy, Steve, Renee and Thomas. It has been great getting to know everyone who has worked in OBEL and the final year and visiting students I've worked with and supervised, Reiner, Cindy, Luke, Julie, Kevin, Lawrence, Oliver, Jonathon T., Jonathon N., Ian, David and Jade.

I am grateful for the Maude Gledden Scholarship which provided the PhD stipend and travel funding.

Finally, I would like to especially thank my family and May for their constant support and encouragement over the years. 



\section{Contents}

$\begin{array}{ll}\text { Abstract } & \text { iii }\end{array}$

Acknowledgements $\quad$ v

Contents vii

List of Figures $\quad$ xi

$\begin{array}{ll}\text { List of Tables } & \text { Xv }\end{array}$

1 Introduction $\quad 1$

1.1 Anatomical optical coherence tomography . . . . . . . . . . . . . 1

1.2 Outline of thesis . . . . . . . . . . . . . . 2

1.3 Selected publications related to this thesis . . . . . . . . . 3

2 Background $\quad 7$

2.1 Sleep apnoea . . . . . . . . . . . . . . . . . . . 7

2.1.1 Physiology of sleep apnoea . . . . . . . . . . . . 7

2.1 .2 Consequences . . . . . . . . . . . . . 8

2.1.3 Prevalence and risk factors . . . . . . . . . . . . . . 9 9

2.1.4 OSA diagnosis and treatment . . . . . . . . . . . . . 10

2.2 Currently available upper airway measurement techniques . . . . . 12

2.3 Motivation for a new technique . . . . . . . . . . . . . . . 15

2.4 Catheter-mounted optical distance ranging probe solution . . . . . 15

2.4 .1 Optical ranging . . . . . . . . . . . . . . . 17

2.4 .2 Time-of-flight ranging . . . . . . . . . . . . 18

2.4.3 Structured light and other non-interferometric methods . . 18

2.4.4 Interferometric methods . . . . . . . . . . . . . . . . 19

2.4 .5 Optical coherence tomography . . . . . . . . . . . . 20

2.4.6 Endoscopic optical coherence tomography . . . . . . . . . . 21

2.5 Summary and conclusions . . . . . . . . . . . . . . 22

$3 \quad$ Key OCT design issues $\quad 23$

3.1 Delay line function and requirements . . . . . . . . . . . 23

3.2 The FDODL . . . . . . . . . . . . . . . . 25

3.2.1 Description of FDODL operation . . . . . . . . . 26 
3.2.2 Other delay lines . . . . . . . . . . . . . . . 27

3.3 The impact of FDODL configuration on achieving a long range scan 28

3.3.1 Description of modelling procedure . . . . . . . . . . 31

3.3.2 Results ..................... 33

3.3 .3 Discussion .................... 34

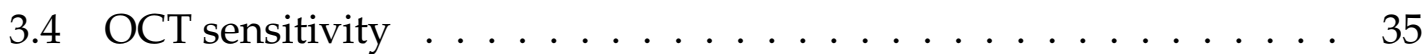

3.4.1 Single-ended configuration ............. 36

3.4.2 Dual balanced configuration . . . . . . . . . . . . 40

3.4.3 Maximising sensitivity of the OCT system . . . . . . . . . 41

3.4 .4 Limited sample power. . . . . . . . . . . . . . . . . 43

3.4 .5 Variable splitting ratio . . . . . . . . . . . . . . 43

3.4.6 Sensitivity-resolution tradeoff . . . . . . . . . . . . . . 44

3.4.7 Fourier-domain OCT . . . . . . . . . . . . . . . 45

3.5 Summary . . . . . . . . . . . . . . . . . . 48

4 System design $\quad 49$

4.1 System layout and interferometer . . . . . . . . . . . . . . . 49

4.2 Sample arm . . . . . . . . . . . . . . . . . 50

4.2.1 Probe design ................... 50

4.2.2 Probe optical characteristics . . . . . . . . . . . 51

4.2 .3 Probe fabrication ................. 53

4.2.4 Probe coil design considerations . . . . . . . . . . . 54

4.2 .5 Catheter ........................ 58

4.3 Electronic signal processing . . . . . . . . . . . . . 58

4.4 Sensitivity and intensity calibration . . . . . . . . . . . . . 59

4.5 Distance calibration . . . . . . . . . . . . . . . 61

4.6 Probe control . . . . . . . . . . . . . . . . . . 61

4.7 Integration with clinical data recorders . . . . . . . . . . . . 62

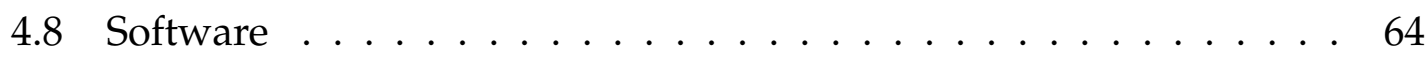

4.9 Lateral resolution of the HOPS images . . . . . . . . . . . . . . 67

4.10 Data processing and presentation . . . . . . . . . . . . 68

4.10 .1 Contour location . . . . . . . . . . . . . . . . 69

4.10 .2 Three-dimensional reconstruction . . . . . . . . . 72

4.11 Summary . . . . . . . . . . . . . . . . . . . 73 
5 Clinical application

5.1 Quantitative upper airway imaging with anatomical optical coherence tomography . . . . . . . . . . . . . . 75

5.2 Abstract ........................... 76

5.3 Introduction . . . . . . . . . . . . . . 77

5.4 Methods . . . . . . . . . . . . . . . . . 78

5.4.1 Description of anatomical optical coherence tomography (aOCT) 78

5.4 .2 Validation studies . . . . . . . . . . . . . . . . . 79

5.4 .3 Feasibility studies . . . . . . . . . . . . 80

5.4 .4 Analyses . . . . . . . . . . . . . . . . . . . 81

5.5 Results ........................... 81

5.5 .1 Validation studies . . . . . . . . . . . . 81

5.5 .2 Feasibility studies . . . . . . . . . . . . 85

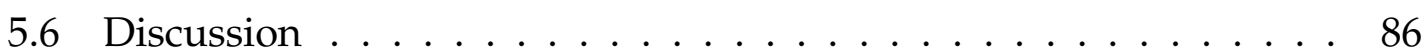

5.6.1 Methodological considerations . . . . . . . . . . 88

5.6 .2 Future applications . . . . . . . . . . . . . . 9 90

5.7 Acknowledgements . . . . . . . . . . . . . . . . . . . . 91

5.8 References . . . . . . . . . . . . . . . . . . 91

5.9 Online data supplement . . . . . . . . . . . . . . . . 94

5.9 .1 Methods ...................... 94

5.9 .2 In vitro validation studies $\ldots \ldots \ldots 96$

5.9 .3 In vivo validation studies . . . . . . . . . . . . . . 97

5.9 .4 References . . . . . . . . . . . . . . . . 98

6 Conclusions $\quad 99$

6.1 Discussion of outcomes . . . . . . . . . . . . . 99

6.2 Future work . . . . . . . . . . . . . . . . . 99

$\begin{array}{ll}\text { Bibliography } & 103\end{array}$

$\begin{array}{lr}\text { A FMCW paper } & 119\end{array}$

A.1 Introduction . . . . . . . . . . . . . . . . . . . . 119

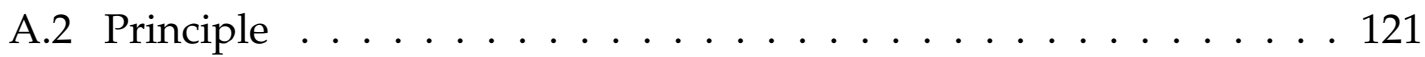

A.3 Experimental system . . . . . . . . . . . . . . . . . 122

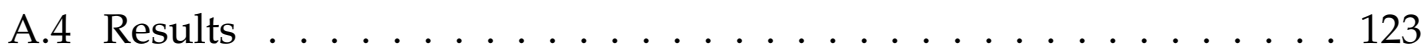

A.5 Discussion and conclusions . . . . . . . . . . . . . . . 125 
$\begin{array}{ll}\text { B Publication list } & 127\end{array}$

$\begin{array}{ll}\text { C FDODL theory } & 133\end{array}$

$\begin{array}{ll}\text { D Basic OCT signal generation theory } & 137\end{array}$ 


\section{List of Figures}

2.1 Basic anatomy of the upper airway. . . . . . . . . . . . 8

2.2 CPAP therapy. . . . . . . . . . . . . . . . . 11

2.3 Examples of mandibular advancement and tongue retaining devices 11

2.4 Tomographic images of the airway. . . . . . . . . . . . . . . . . 14

2.5 The catheter and probe in the upper airway. . . . . . . . . . . . 16

2.6 In vivo intravascular OCT images of (left) rabbit aorta and (right) human coronary artery. . . . . . . . . . . . . . . . 22

3.1 Bar graph of retropalatal (RP), retroglossal (RG) and epiglottal (EPG) pharyngeal measurements . . . . . . . . . . . . . . 25

3.2 The FDODL operates by tilting the galvanometer mirror. . . . . . . 27

3.3 In-plane FDODL configuration. . . . . . . . . . . . . . . . 28

3.4 In-plane PBS-based FDODL configuration. . . . . . . . . . . . . 31

3.5 Out-of-plane FDODL configuration. . . . . . . . . . . . . . . . 31

3.6 (a) "on-pivot" and (b) "off-pivot" FDODL configurations . . . . . . 32

3.7 ZEMAX model views . . . . . . . . . . . . . . . . . . . 33

3.8 Results of the ZEMAX modelling. . . . . . . . . . . . . . . . . . 34

3.9 Output spectrum of the FDODL versus delay. . . . . . . . . . . . . . 35

3.10 SNR definition. . . . . . . . . . . . . . . . . . . 36

3.11 (a) Single-ended OCT interferometer and (b) dual balanced OCT interferometer. . . . . . . . . . . . . . . 36

3.12 OCT sensitivity for the (a) single-ended configuration and (b) dual balanced configuration. . . . . . . . . . . . . . . . 42

3.13 OCT sensitivity versus source power. . . . . . . . . . . . . . . . 42

3.14 OCT sensitivity versus source power. . . . . . . . . . . . . 43

3.15 Sensitivity versus splitting ratio . . . . . . . . . . . . . . . . . 44

3.16 Optimum sensitivity versus source power for single-ended and dual-balanced configurations . . . . . . . . . . . . . 45

3.17 Simulated OCT depth resolution and sensitivity penalty versus the ratio of filter to signal bandwidth. . . . . . . . . . . . . . . . . 46

4.1 Schematic diagram and photograph of the aOCT system. . . . . . . 49

4.2 A diagram and photograph of the probe optics. . . . . . . . . . . . . 51

4.3 Plots for determining the GRIN lens length to achieve a maximum distance scanning response. . . . . . . . . . . . . . . . . . . 52 
4.4 Wave/ray tracing simulation of probe coupling efficiency. . . . . . 54

4.5 Measured probe performance at three steps during the final lens length adjustment. . . . . . . . . . . . . . 55

4.6 Measured probe coupling efficiency versus distance. . . . . . . . . . 55

4.7 Two key torque coil properties for the aOCT probe - response to rotation and translation. . . . . . . . . . . . . . . 56

4.8 aOCT images of a square tube for a set of curved catheter paths. . . 57

4.9 Geometry used to calculate the minimum bend radius for a probe and catheter characteristics. . . . . . . . . . . . . . . . . . . 59

4.10 Schematic diagram of the signal processing electronics. . . . . . . . 60

4.11 An example of typical data from a single A-scan. . . . . . . . . . . . 60

4.12 Example of sensitivity calibration measurement. . . . . . . . . . . 61

4.13 Example of distance calibration measurement. . . . . . . . . . . . . 62

4.14 CAD model of the probe control subsystem. . . . . . . . . . . . . 63

4.15 The synchronization outputs from the aOCT system. . . . . . . . . . 64

4.16 Schematic of the $a$ OCT software . . . . . . . . . . . . 65

4.17 Front panel screenshot . . . . . . . . . . . . . . . . 65

4.18 Process of rendering of polar-encoded data to Cartesian-based display field. . . . . . . . . . . . . . . . . . . 67 67

4.19 Transverse resolution of the HOPS images . . . . . . . . . . . . . 68

4.20 Screenshots of typical video files used for later review of data by the clinician. . . . . . . . . . . . . . . . . . . . 69

4.21 Example of contour successfully drawn using the virtual robot approach. ........................ 72

4.22 Example of GVF active contours converging on an airway boundary. 72

4.23 Three-dimensional model created from a $20 \mathrm{~mm}$ segment of a pullback data set. . . . . . . . . . . . . . . . . . . 73

5.1 Bland-Altman plot of the agreement between measurements of a wax phantom. . . . . . . . . . . . . . . 82

5.2 Selected images from a 'pullback scan' of a subject without sleep apnea. ...................... 83

5.3 Simultaneous $a$ OCT scans and CT scans obtained in the hypopharynx, oropharynx and velopharynx. . . . . . . . . . . .

5.4 Bland-Altman plots of the agreement between measurements in 5 healthy subjects. . . . . . . . . . . . . . . . . . . . . 84 
5.5 The effect of changing airway pressure on maximum and minimum cross-sectional area. . . . . . . . . . . . . . . . . . 86

5.6 Changes in upper airway caliber before, during and after airway collapse. . . . . . . . . . . . . . . . . 87

A.1 A schematic of the distance ranging system. . . . . . . . . . . . . 122

A.2 Example of (a) an unprocessed photocurrent waveform, and (b) the corresponding normalised waveform. . . . . . . . . . . . . 123

A.3 Distance ranging to a sample of bovine muscle tissue. . . . . . . . . 124

A.4 Position resolution versus sample return loss. . . . . . . . . . . . . 124

C.1 Schematic of the FDODL. . . . . . . . . . . . . . . . . 133

C.2 Photograph of the FDODL as implemented. . . . . . . . . . . . 136

D.1 Simple OCT inteferometer . . . . . . . . . . . . . . . . 138

D.2 Example of OCT fringes . . . . . . . . . . . . . . . . . . 139 



\section{List of Tables}

3.1 Maximum velopharyngeal airway dimensions measured by Ciscar et al. . . . . . . . . . . . . . . . . 24

3.2 Parameters of reported frequency-domain optical delay lines. . . . 27

3.3 A survey of other delay line designs. . . . . . . . . . . . . . . . . . 29 


\section{Introduction}

\subsection{Anatomical optical coherence tomography}

The purpose of this PhD thesis is to develop and assess a new technique for the in vivo dynamic measurement of the size and shape of the human upper airway. The initial medical application is the investigation of obstructive sleep apnoea (OSA). The device developed is a transnasal pharyngeal catheter-mounted probe utilising an optical interferometric technique, optical coherence tomography (OCT), to perform distance ranging in the upper airway, with the aim of twoand three-dimensional reconstruction of the upper airway profile. The technique is referred to as "anatomical optical coherence tomography" (aOCT).

Although the condition of OSA has long been medically recognized, it has more recently been identified as a condition associated with significantly increased morbidity and mortality, a high social cost, and affecting a significant fraction of the population ( $2-4 \%$ of middle aged people). In recent years medical research into OSA has been greatly advanced; however, there are many as-yet unknown aspects of the pathophysiology, and many of the currently-used treatment options still have low rates of success. Currently, there does not exist any practical method by which quantitative in vivo measurements of cross-sectional area and profile of the human upper airway can be routinely and safely acquired during accurately characterized sleep. Exising technologies can provide accurate measurements of the airway during wakefulness but results suggest that measurements taken while subjects are awake are not sufficient for understanding the airway behaviour during sleep. The tool developed is intended to take these measurements during sleep, greatly assisting researchers in identifying the precise mechanism of airway collapse in patients with OSA. The ability to perform quantitative measurements will allow studies to be carried out to identify correlations in airway anatomy and dynamical airway behaviour. This should lead to a better understanding of the pathophysiology of OSA, leading to improved treatments for sufferers of the condition. The measurements provided by the new device will be also be used in accurately identifying sites of airway collapse in individuals, which will assist in the selection of the most appropriate therapy for sufferers of OSA.

The nasally-inserted catheter is transparent and remains stationary during measurements, the distal end of the catheter anchored in the oesophagus by inherent oesophageal peristalsis. The probe inside the catheter can be rotated and 
translated without disturbing the subject, and in the typical imaging modes the probe either rotates at a certain location to capture an airway shape measurement in a single cross-sectional plane, or simultaneously translates and rotates to capture a helical data set. The OCT system used is the time-domain form of OCT, and the system is unique amongst reported OCT systems in employing a very long range optical delay line. Software interfaces and processing tools were created to have full and user-friendly control of the aOCT instrument in a clinical setting, and also offline programs written to correlate data streams with other recorded physiological measurement data (e.g. air flow rate, EEG, ECG signals) and analyze/present the resulting data sets. A published research article demonstrated some of the potential for clinical measurements. Helical scanning of the airway from the hypopharynx to the nasal cavity has been demonstrated, as has capability to monitor a location for changes under external stimuli such as applied air pressure. Initial efforts towards three-dimensial reconstruction of the airway useing the data have been demonstrated. Finally, the system was used in cooperation with standard polysomnography to take quantitative measurements of airway shape and size and during the sleep and apneic episodes of a severe sleep apneic, which is the first reported example of this capability.

\subsection{Outline of thesis}

Chapter two of this thesis is concerned with the motivation for and introduction of the new airway measurement technique as a tool to assist in research into sleep apnoea. Sleep apnoea is first introduced, then a summary is given of the existing technologies for imaging/measuring the part of the airway where sleep apnoea collapse occurs. The concept of the new optical-ranging endoscopic technique for airway measurement is described, and existing potential opticalranging technologies are surveyed. The chosen technology, optical coherence tomography (OCT), particularly endoscopic OCT, is introduced.

In the third chapter two technical issues of importance to the aOCT system are discussed; the OCT optical delay line and the OCT system sensitivity. The delay line employed by the aOCT system is significantly longer than those used in other OCT applications, and optical modelling was carried to examine some of the design choices involved in the use of the frequency-domain optical delay line (FDODL). The signal levels and noise sources are analysed and the sensitivity performance of the OCT system is optimised.

The following chapter deals with other engineering issues involved in build- 
ing the portable aOCT system for use in the clinic. The system hardware is described, particularly the optical design and construction of the probes. The system software, integration with other sleep laboratory recording equipment, and data processing and presentation are covered.

The fifth chapter consists of a research paper published in the American Journal of Respiratory and Critical Care Medicine (see [XII] below). The paper is concerned with the validation of $a \mathrm{OCT}$ and examples of clinical measurements; full scanning of the entire pharynx, measurement of airway compliance, and measurement of airway behaviour during apnoea events (prolonged airway collapse during sleep). The final chapter contains conclusions and summary of future work.

The first appendix contains a published paper (see [I] below) which describes an optical ranging technique first intended to be used for the aOCT system but later replaced by time-domain optical coherence tomography. This technique may in future be used if suitable commercially-produced tunable lasers become available. The second appendix provides a list of all publications that I have coauthored during this project.

\subsection{Selected publications related to this thesis}

Selected refereed and invited publications (note: includes one invited book chapter still in press, one paper currently under review) relating to $a \mathrm{OCT}$ and its development are listed below. PDFs and online multimedia material of some of these publications are available on the website of the Optical+Biomedical Engineering Lab (OBEL):

http://obel.ee.uwa.edu.au/publications/

[I] J. J. Armstrong, D. D. Sampson, “Distance ranging to biological tissue using fibre-optic Fabry- Perot, short tuning range FMCW interferometry" in 14th International Conference on Optical Fiber Sensors, Florence, Italy, A. G. Mignani, H. C. Lefevre, Editors, Proc. of SPIE, vol. 4185, pp. 366-369, 2000.

[II] D. D. Sampson, B. A. Patterson, J. J. Armstrong, "Some biomedical applications of optical fibre sensors," in Proc. of Optoelectronics and Communications Conference/Integrated Optics and Optical Fibre Communications Conference, pp. 482-485, 2001 (invited). 
[III] D. D. Sampson, A. V. Zvyagin, J. J. Armstrong, "Some applications of optical coherence tomography," in Focus on Microscopy, Technical Digest, pp. 27, Kaohsiung, Taiwan, April 2002 (invited).

[IV] J. J. Armstrong, M. S. Leigh, I. D. Walton, A. V. Zvyagin, S. A. Alexandrov, S. Schwer, D. D. Sampson, D. R. Hillman, P. R. Eastwood, "In vivo size and shape measurement of large hollow organs using endoscopic long-range optical coherence tomography", Optics Express, vol. 11, no. 15, pp. 18171826, 2003.

online journal link: http: / / www . opticsexpress . org/abstract . cfm? URI $=$ OP EX $-11-15-1817$

[V] J. J. Armstrong, M. S. Leigh, D. R. Hillman, P. R. Eastwood, D. D. Sampson, "In vivo size and shape measurement of the human upper airway using an endoscopic long-range OCT system," presented at Advances in Optics for Biotechnology, Medicine and Surgery, Poster 4, Banff, Alberta, Canada, August 2003.

[VI] J. J. Armstrong, M. S. Leigh, I. D. Walton, S. Schwer, A. V. Zvyagin, S. A. Alexandrov, D. D. Sampson, D. R. Hillman, P. R. Eastwood, "An endoscopic long-range optical coherence tomography system and its application to in vivo size and shape measurement of large hollow organs," in 16th International Conference on Optical Fiber Sensors, Nara, Japan, K. Hotate, H. Nagai, Editors, pp. 722-725, October 2003.

[VII] D. D. Sampson, A. V. Zvyagin, E. D. J. Smith, I. D. Walton, J. J. Armstrong, K. K. M. B. D. Silva, “The frequency-domain delay line in optical coherence tomography: design, capabilities, and limitations," in Conference on Lasers and Electrooptics-Pacific Rim (CLEO-PACRIM), Taiwan, paper W2E-(12)-1, July 2003 (invited).

[VIII] P. R. Eastwood, J. J. Armstrong, A. V. Zvyagin, I. Walton, S. A. Alexandrov, S. Schwer, M. S. Leigh, D. D. Sampson, D. R. Hillman, “In vivo size and shape measurement of the upper airway using endoscopic optical coherence tomography (OCT)," in 14th European Respiratory Society Annual Congress, European Respiratory Journal, vol. 24 (Supplement 48): P2743, September 2004.

[IX] D. D. Sampson, J. J. Armstrong, M. S. Leigh, D. R. Hillman, P. R. Eastwood, 
"Imaging human upper airway architecture dynamically using optical coherence tomography," in 8th International Conference on Optics Within Life Sciences, Melbourne, Australia, pp. 143-144, November 28-December 1, 2004 (invited).

[X] P. R. Eastwood, J. J. Armstrong, M. S. Leigh, A. Paduch, D. D. Sampson, J. H. Walsh, D. R. Hillman, "Assessing upper airway structure and function with anatomic optical coherence tomography (aOCT)," in Annual Scientific Meeting of the Australasian Sleep Association, Gold Coast, QLD, Australia, 7-9 October, 2005 (invited).

[XI] M. S. Leigh, J. J. Armstrong, A. Paduch, D. D. Sampson, J. H. Walsh, D. R. Hillman, P. R. Eastwood, "Quantitative imaging of the human upper airway: instrument design and clinical studies" in Saratov Fall Meeting: Optical Technologies in Biophysics \& Medicine VII, (V. V. Tuchin, ed.), Saratov, Russia, September 2005 (invited).

[XII] J. J. Armstrong, M. S. Leigh, D. D. Sampson, J. H. Walsh, D. R. Hillman, and P. R. Eastwood, "Quantitative Upper Airway Imaging with Anatomic Optical Coherence Tomography," American Journal of Respiratory and Critical Care Medicine, vol. 173, pp. 226-233, 2006.

online journal link: http://ajrccm. ats journals.org/cgi/content/ abstract/173/2/226

[XIII] M. S. Leigh, J. J. Armstrong, A. Paduch, J. H. Walsh, D. R. Hillman, P. R. Eastwood, D. D. Sampson, "Anatomical Optical Coherence Tomography: Instrument design and application to the upper airway," IEEE Transactions on Medical Imaging (submitted).

[XIV] J. J. Armstrong, M. S. Leigh, D. R. Hillman, P. R. Eastwood, and D. D. Sampson, "Anatomical Optical Coherence Tomography of the Human Upper Airway," in "Optical Coherence Tomography: Technology and Applications," editors Wolfgang Drexler and James G. Fujimoto, to be published by Springer (invited, in press). 



\section{Background}

In this chapter the motivation for a new airway measurement instrument and an introduction to the instrument technology are presented. Firstly a short description of the pathophysiology of sleep apnoea is given. Then a brief review of the medical literature is presented highlighting the consequences of sleep apnoea, the high level of prevalence in the population, and a review of the typical diagnosis and treatment options. This is followed by a brief description of the currently used airway measurement techniques, including the shortcomings which have created the need for a new instrument. The concept of airway structure measurement with an optical probe in a naso-oesophageal catheter is presented followed by its implementation using an optical reflectometry technique, optical coherence tomography. Current uses of optical coherence tomography are discussed.

\subsection{Sleep apnoea}

Sleep apnoea is a condition in which breathing is interrupted during sleep (apnoea is derived from the Greek "without breath"). It is characterized by repetitive interruption of normal breathing causing periods of hypoxaemia and a fragmented sleep architecture. Sleep apnoea may be classified as obstructive sleep apnoea (OSA) or central sleep apnoea (CSA). OSA involves a physical obstruction to air flow and is the most common form of sleep apnoea. CSA is a neurological condition in which all respiratory effort is temporarily absent, and is relatively rare.

A sleep apnoea event is defined as one in which breathing is interrupted for a duration of 10 seconds or longer. A hypopnea event is an episode of reduced air flow and blood oxygen saturation. The apnoea-hypopnea index (AHI) is the number of apnoea or hypopnea events per hour. A person is usually said to be a sleep apnoea sufferer if they have an AHI of five or more. They are said to suffer from obstructive sleep apnoea-hypopnea syndrome (OSAS) if they also have associated excessive daytime fatigue or sleepiness.

2.1.1 Physiology of sleep apnoea Figure 2.1 is a diagram of the airway anatomy relevant to sleep apnoea. The key structure is the pharynx, which links the mouth and nose to the trachea and oesophagus. The pharynx is a compliant and therefore collapsible tube, normally kept open by the pharyngeal dilator muscles. Reduction of the function of these dilator muscles increases the vulnerability of the pharynx to collapse. The activity of most skeletal muscles is reduced during 
all stages of sleep and whilst in the rapid eye movement (REM) phase nearly all of these muscles, including the pharyngeal dilators, are paralysed. The only exceptions are the diaphragm muscles and the extraocular muscles (which generate the eye movements that the sleep phase is named after). Non-skeletal muscles such as the heart and gastric muscles are not affected. For a variety of reasons, the airway may be narrow at certain points and thus the cross-sectional area available for the airflow is reduced. This causes a relative increase in air speed, reduction in pressure (Bernoulli Principle) and possibly increased air turbulence. Without the compensatory effect of the dilator muscles the airway may then collapse, causing partial or complete closure. After collapse, the flow of air to the lungs is reduced, causing blood oxygen saturation to begin to fall below normal levels and dissolved $\mathrm{CO}_{2}$ levels to rise. Respiratory effort, in the form of diaphragm muscle movement, continues during the period of interrupted breathing. After some time the increased $\mathrm{CO}_{2}$ levels trigger an automatic response involving partial arousal so that the dilator muscles are activated and re-establish airway patency. This series of events may occur repetitively during the night; for example 50-100 episodes per hour would be considered serious but not highly unusual.

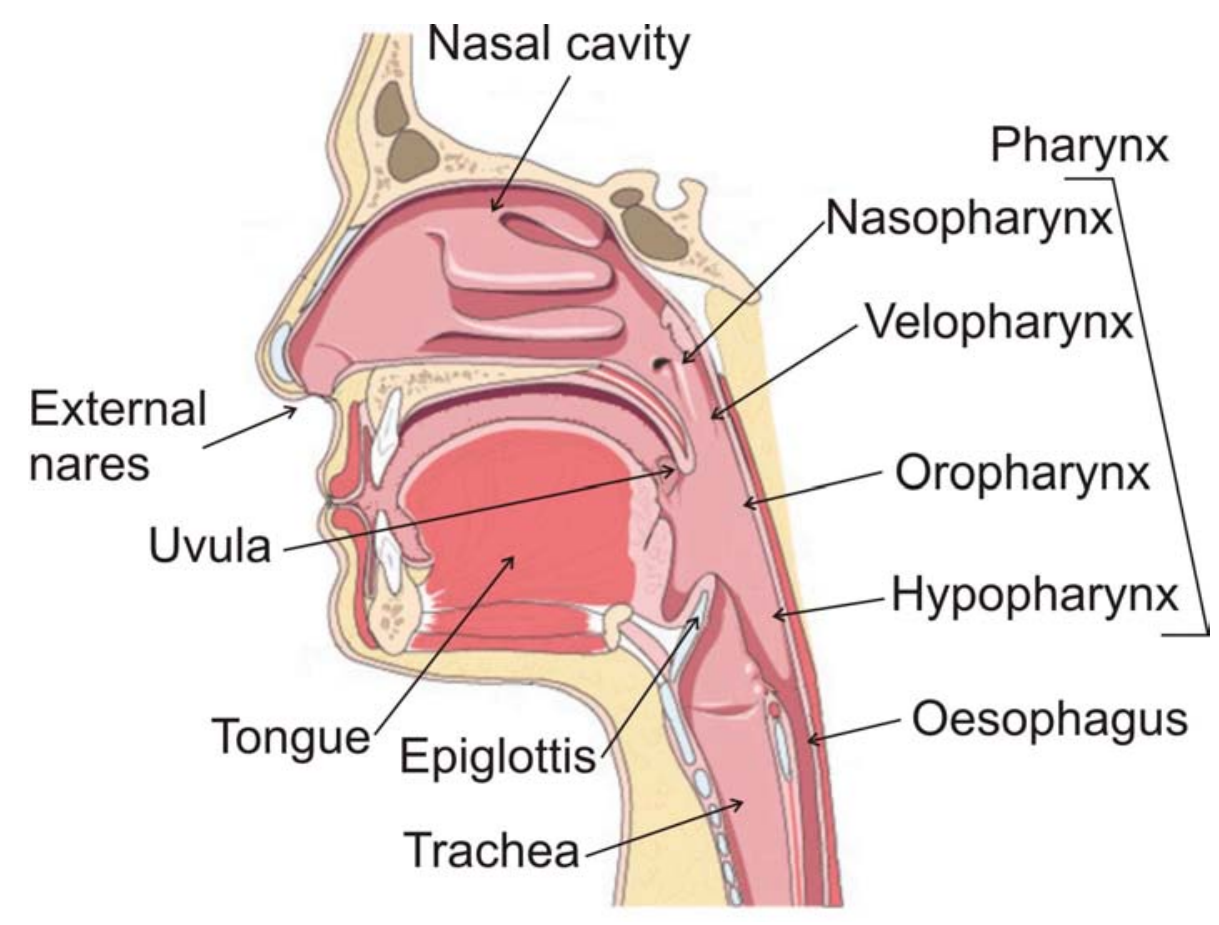

Figure 2.1: Basic anatomy of the upper airway.

2.1.2 Consequences Many negative consequences of OSA for health and wellbeing have been discovered. Young states that: "In statistical studies OSA 
has been shown to be independently associated with increased likelihood of hypertension, cardiovascular disease, stroke, excessive daytime sleepiness, motor vehicle accidents, and diminished quality of life" [1]. REM sleep is repeatedly interrupted by OSA. This has severe consequences as the REM phase is an essential component of normal sleep. Rats, with a normal lifespan of 2-3 years, on average survive 5 weeks when deprived of only REM sleep, and only 3 weeks when deprived of all sleep stages [2]. They also suffer reduced nervous system function and a depressed immune system response, as do humans under sleep deprivation $[3,4]$. Human beings can survive for upwards of a month without food but less than 2 weeks without sleep (total sleep deprivation).

A number of studies have suggested that sleep apnoea sufferers have a greater risk of death by cardiovascular or cerebrovascular events, or motor vehicle accidents. Sleep apnoea is a risk factor for morbidity and mortality from heart attacks $[5,6]$. Evidence has been presented for a causal link between sleep apnoea and systemic hypertension [7]. A study of 210 subjects found a factor of three increase in the rate of motor vehicle collisions as compared to control subjects [8], and subsequently the rate fell to the same as the control subject rate with the use of continuous positive applied pressure (CPAP) treatment. Another study found that an AHI greater than 15/hour increased the chance of multiple motor vehicle accidents by 7.3 [9]. Correlations have been identified between sleep apnoea and sudden infant death syndrome [10], and poor school performance [11].

As well as consequences regarding the health and wellbeing of individuals, the impact of OSA on society is considerable. The economic impact in Australia of sleep disorders, including OSA, has been estimated to be $\$ 200 \mathrm{~m}$ in direct health costs and $\$ 10.3 \mathrm{~b}$ in economic costs [12]. In the US, research has estimated an annual cost of US\$15.9b for OSA-related motor vehicle accidents [13]. In addition, several large industrial accidents around the world have been attributed to fatigued and possibly sleep apnoeic workers, e.g., the Exxon Valdez oil spill and the Three Mile Island and Chernobyl nuclear power plant accidents [14].

2.1.3 Prevalence and risk factors Several large epidemiological studies (Wisconsin [15], Pennsylvania [16], Spain [17]) have been carried out to examine OSA prevalence, and to discover associations between OSA and parameters such as age, sex and body mass index. In the U.S.A. up to $5 \%$ of adult males suffer from OSAS [1]. The estimated proportion of the population that suffers from OSA alone (i.e. AHI > 5/hour) is much higher $-24 \%$ for men and $9 \%$ for women, of middle age (30-60 years). Similarly, the rate amongst Australian adult males 
with an $\mathrm{AHI}>5 /$ hour and daytime sleepiness has been found to be a minimum of $3 \%$, and $26 \%$ have an AHI $>5 /$ hour [18]. OSA has been found to be usually unrecognized and undiagnosed [1]. The likely reason is that usually an OSA sufferer is not directly aware of the sleep interruptions as they do not experience full arousal. The estimated prevalence of constant snoring in children is approximately $8-9 \%$ and of sleep apnoea is $1-2 \%$. Male to female ratio is 1:1 (cf. $4: 1$ in adults).

Certain factors have been found to be associated with OSA, some genetic and some environmental. These include obesity (an increasing problem in Western countries), smoking, excessive alcohol consumption, tonsillar hypertrophy, postmenopausal estrogen reduction and physiological genetic predisposition [1].

2.1.4 OSA diagnosis and treatment OSA is conclusively diagnosed by an overnight sleep study (polysomnography) in a sleep clinic. Polysomnography is used to monitor a range of physiological parameters, including respiratory flow and effort, blood oxygen saturation, heart rates (ECG), neurological sleep state (EEG), level of snoring (sound measurement), and limb movement in accordance with an international standard [19]. From these data a diagnosis of OSA and its severity can be made. With this information and knowledge of other patient symptoms, such as daytime fatigue and sleepiness, a sleep specialist will prescribe treatment. In severe cases the physician may request surrender of the patient's driver's license and recommend leave from work if it is necessary for public safety (e.g. an airport flight controller).

Currently, the most effective and commonly-used treatment continuous positive applied pressure (CPAP) therapy [20]. As shown in Figure 2.2 this involves a nasal mask connected to a bedside machine by an air hose. The machine supplies a continuous flow of air to the mask at a preset pressure, greater than atmospheric pressure. More sophisticated versions vary the pressure based on the air flow and breathing rate. CPAP provides a pneumatic splint to maintain airway patency (openness) during sleep. Whilst CPAP is an extremely effective treatment, it is not a cure, and some patients do not tolerate it well, with complaints such as:

- Dry nose, nosebleeds, and sore throat

- Nasal congestion, runny nose, and sneezing

- Irritation of the eyes and the skin on the face

- Abdominal bloating 
- Headaches

- Nightmares and excessive dreaming

Rare complications include severe nosebleeds and bacterial infection in the lining around the brain and spinal cord (meningitis).
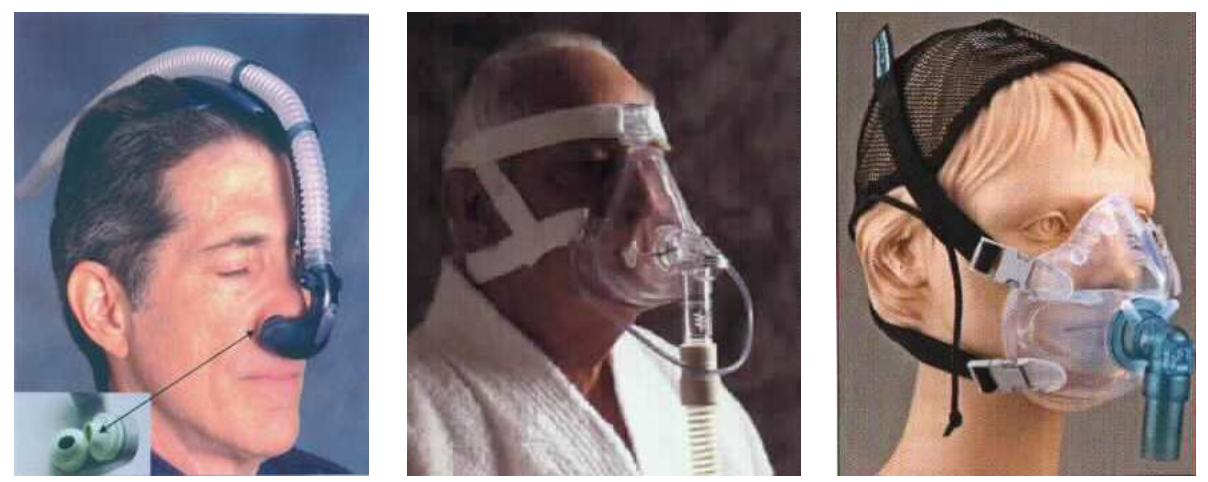

Figure 2.2: CPAP therapy is the most commonly used therapy for OSA (image source: http://www. cpapman.com/ffmasks.html).

Dental/oral device OSA treatments are also popular. The two common classes are mandibular advancement devices (MAD) and tongue advancement devices (TAD). These work by attempting to alter the gross anatomy of the pharynx in an effort to reduce the airway collapsibility. There is a large range of MADs and TADs commercially available; see Figure 2.3. The treatments are less effective than CPAP overall, however, they work well for a subset of OSA patients. It is not clear before treatment commences which patients will experience positive results. This is unfortunate as often the devices are custom made by a specialist dentist and relatively expensive.
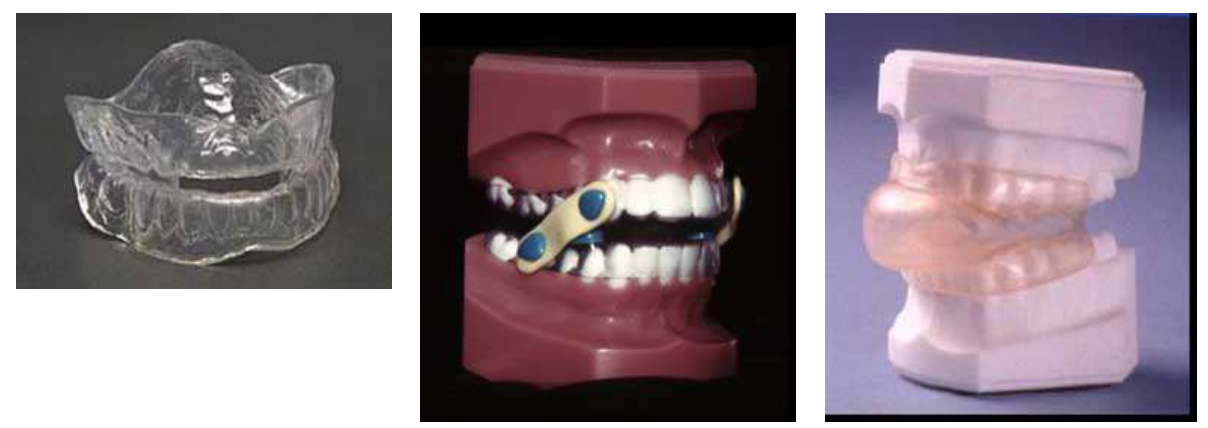

Figure 2.3: Examples of mandibular advancement and tongue retaining devices (image source: http://www. quietsleep.com).

The various surgical options include altering the characteristics of the tissue of the upper airway, and the resection (removal) and reshaping of upper airway tis- 
sue to increase airway size. To alter the tissue properties, heat is usually applied via electrical current, or microwave/optical radiation. This makes the airway tissue less compliant. However, outcomes are often uncertain and long term success rates are less than 50\%. Ryan et al. found that: "The treatment response to LAUP (laser-assisted uvulopalatoplasty) is variable and unpredictable, and only a few patients achieve a satisfactory response. There appears to be no relationship between subjective and objective measures of treatment efficacy." [21]. In this study, more than $30 \%$ of patients had worse breathing disturbance after LAUP. Some physicians are also concerned that if LAUP eliminates snoring, then a diagnosis of apnea may be missed in patients who have the more serious condition.

The most common form of resection surgery is uvulopalatopharyngoplasty (UPPP), which has been used as a treatment for OSA since it was first described in 1981 [22]. The procedure involves the removal of tissue around the nasopharynx and oropharynx, typically large amounts of the soft palate and uvula. However, the operation is not free from risks and the success rate has been found to be only $40 \%$ [23]. Common side effects and complications from UPPP include pain, alteration in taste and smell, change in voice frequency, the sensation of a foreign body while swallowing, and the degradation of the "valve" function of the soft palate. This can result in excess nasal tones while talking and leakages of liquids from the nose (nasal regurgitation). Also, the tissue removal may later reduce the efficacy of CPAP treatment - which is very unfortunate for those patients with an unsuccessful UPPP outcome. In cases of life-threatening OSA, tracheostomy (creation of an opening through the neck into the windpipe) is an option with almost $100 \%$ success. $20 \%$ of patients who had UPPP subsequently required a tracheostomy. However, it is a radical treatment and a daytime plug must be worn in the tracheal opening to allow normal talking.

\subsection{Currently available upper airway measurement techniques}

Over the last three decades there has been a considerable amount of research into the pathogenesis and treatment of OSA. Researchers have striven to gain a fuller understanding of the causes and characteristics of airway collapse by investigating the static and dynamic properties of the airway structure. These have been investigated using a variety of measurement techniques which can be divided into imaging and non-imaging techniques. Imaging techniques include:

- cephalometric radiographs (static x-ray images) 
- radiographic computed tomography (CT) imaging,

- magnetic resonance imaging (MRI),

- x-ray fluoroscopy (a time-series of two-dimensional x-ray images),

- direct visualisation using video endoscopy.

Non-imaging techniques:

- air pressure measurement at a series of points along the airway,

- acoustic reflection,

- various cranio-facial lengths and angles (known as cephalometric measures), for example, mandibular length.

Unfortunately, none of these techniques has provided researchers the ability to repeatedly, safely and accurately measure the shape and size of the upper airway during sleep.

The non-imaging techniques provide some useful information but do not accurately measure airway shape. Acoustic reflection [24] provides an estimate of the location of collapsed segment of airway, but does not examine the nasopharynx, which is a common site of upper airway collapse. Also, most patients occlude their airway over a significant length $[25,26]$, but the technique can only detect the most proximal site of obstruction [27]. Cranio-facial measurements only provide indirect information about the shape of the pharynx; they provide no clue as to dimensional changes occurring in the airway during collapse. Nocturnal esophageal catheter pressure measurement allows the site of collapse to be localized, and also detects respiratory effort, however lumen size information is not available.

Direct visualization, using video endoscopy in the airway, is not suitable for making quantitative measurements. Manual and automatic outlining of the airway wall is subjective and inaccurate, and strongly influenced by the position of the fibre-optic scope [28]. The optical field is distorted due to the small aperture imaging lens in the endoscope. CT and MRI have been used to take accurate measurements of airway structure [29-34] but these are not necessarily representative of the pharynx during sleep and particularly episodes of collapse. Neither technique is suitable for lengthy evaluations or evaluations during validated sleep [35]. The claustrophobic and noisy environment makes it difficult 
for an unsedated patient to sleep in the machines. Sedation has been used to induce sleep for MRI measurements of airway dimensions [36], though sedation is known to affect the apnoea condition. Due to the large magnetic fields in MRI it is not possible use metallic probes and leads to measure the physiological parameters which are necessary to determine the state of sleep (e.g. electroencephalography and muscle activity measurements). X-ray imaging techniques (radiographs, CT, fluoroscopy) have radiation-dose limitations so cannot be used over repeated or extended time periods. As an example, Figure 2.4 shows CT and MRI images of the airway used for sleep apnoea research. These measurements, while useful, cannot be performed simultaneously with polysomnography. MRI and CT machines are also expensive to access; this makes routine use for sleep apnoeics and large scale studies less feasible.

(a)

(b)

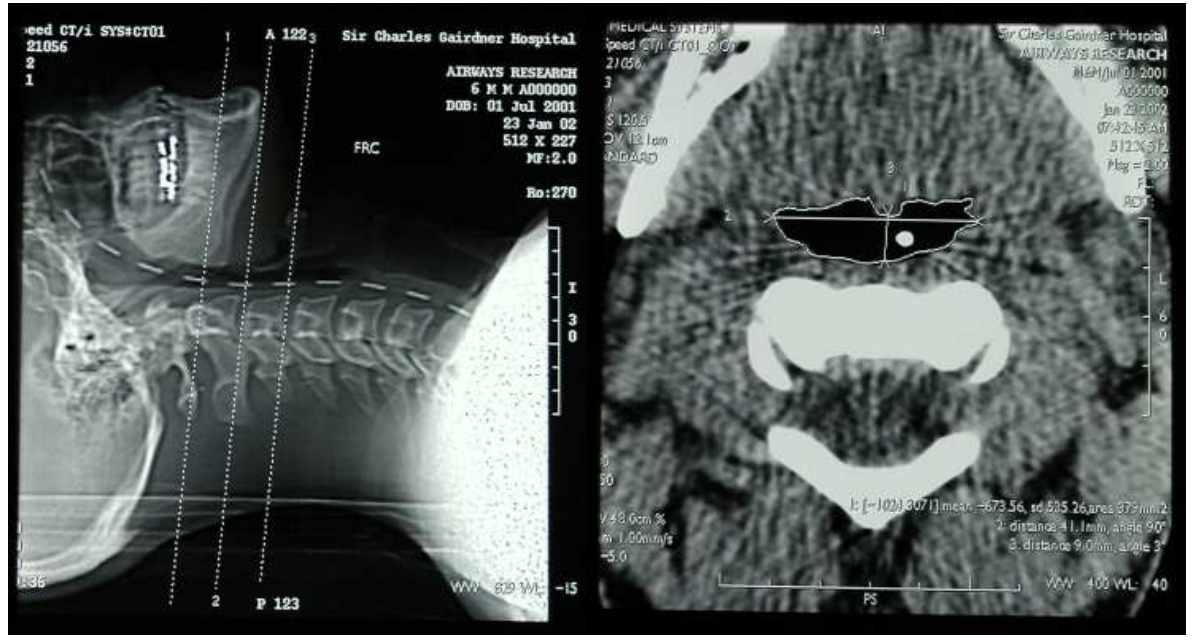

(c)

(d)

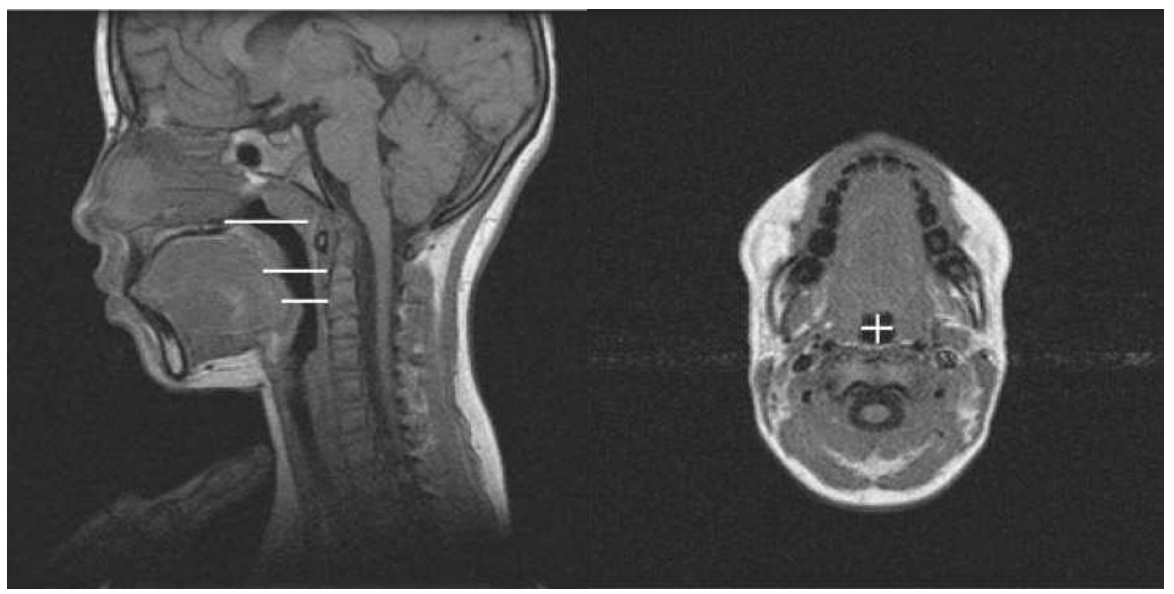

Figure 2.4: Tomographic images of the airway using (a), (b) x-ray CT (source: Sir Charles Gairdner Hospital) and (c), (d) MRI (source: [37]). 


\subsection{Motivation for a new technique}

The above techniques have been used in attempts to predict the site of obstruction in individuals [38]. However, the results have been highly variable [38,39], suggesting that muscle activity in the pharynx can obscure abnormalities in airway function which cause sleep-disordered breathing. Also, measurements taken during wakefulness are poor indicators of the success rate in surgical treatments $[39,40]$.

Results suggest that measurements taken while the subject is awake are not sufficient for understanding the airway behaviour during sleep. Certainly the low success rates of the current OSA treatments other than CPAP indicate the potential for better selection of treatment options to improve treatment success.

As the sites of collapse in individual patients are unspecified the current treatments are relatively crude. They either work on the principle of distending the entire pharynx to reduce collapsibility, or surgical modification of locations such as the soft palate, without reference to data on the individual. It is believed that identification of the site(s) of upper airway collapse during sleep is vital to understanding the pathogenesis of OSA and devising treatments for it. Thus, a new technique capable of making accurate measurements of the airway shape and size could enable improved targeting of existing surgical and mechanical treatments, and the creation of new treatments.

\subsection{Catheter-mounted optical distance ranging probe solution}

Based on the requirements of safe, real-time and accurate airway measurement, an optical distance ranging catheter-mounted probe was proposed. As shown in Figure 2.5, a transparent catheter is passed through the external nares and pharynx, with the end located in the oesophagus where it is held by peristaltic action. An optical probe inside the catheter uses light to measure the distance to the airway wall, that is, the interface between air and tissue. The light beam is perpendicular to the catheter axis as shown in the figure. The probe can be systematically rotated and translated along the catheter to collect distance data over the airway. From this the airway size and shape can be dynamically measured.

Previous studies have demonstrated that placement of such a catheter in the upper airway and oesophagus does not induce additional sleep disturbance in patients with sleep-related breathing disorders [41,42]. From discussions with airway physiologists, $3 \mathrm{~mm}$ was decided as a reasonable initial target for the 
catheter tubing diameter to maintain patient comfort and not interfere with the sleep pattern.

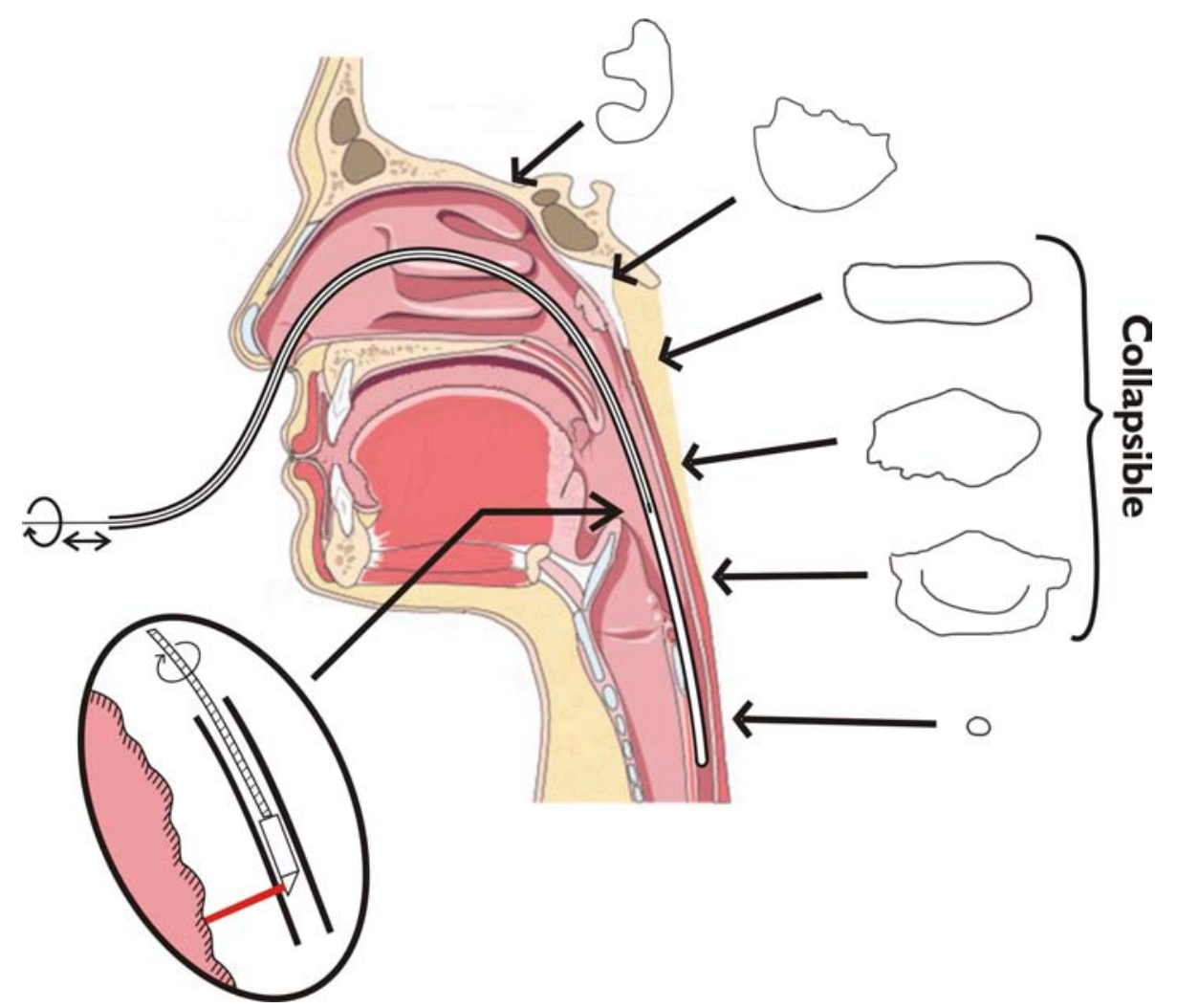

Figure 2.5: The catheter and probe in the upper airway. The line images on the right are example cross-sectional profiles of the pharynx and oesophagus around the probe.

To provide accurate airway measurements the probe should take measurements fast enough to avoid motion artifacts. The causes of motion in the airway would typically be breathing and airway collapse. Swallowing and head/neck movement also cause changes in airway shape but these can be controlled during an experiment. The average breathing rate for adults who are awake and at rest is 12 to 14 breaths per minute. The average decreases to 6 to 8 per minute during sleep. At an image rate of four complete rotations per breath, $1 \mathrm{~Hz}$ rotation would be sufficient, assuming 15 breaths per minute. However, the actual event of airway collapse occurs during a fraction of a breath, which is known from observation of measurements of flow and airway pressure. The rate of imaging required to observe a collapse is currently not known precisely; this is an area which could be investigated further with a working system.

Another potential source of motion artifacts is the beating of the heart which, at $60-80 \mathrm{~Hz}$ for an adult at rest, is much faster than the probe rotation rate. As in 
the forseeable future there are no plans to scan the airway with a probe rotation rate faster than this, it does remain a possible source of artifacts. However, we are currently not aware of any reported detection of movement in the airway due to the pumping of the heart. If for some reason this did become a significant source of error, then it should be possible to simultaneously sample the heart rate - in fact this is already part of the standard measurement set for sleep studies and use the information to correct for the artifacts. A similar procedure has been carried out in the field of intravascular ultrasound [43].

The required resolution of measurements in the airway needs to comparable to existing technologies such as CT and MRI. The actual resolution achieved by these modalities depends on a range of imaging parameters which are set depending on the particular requirements of each experiment. It is preferable to avoid exposure to high levels of $x$-rays, so the images produced are not of the maximum resolution of which the machine is capable. It is our observation that approximately $1-2 \mathrm{~mm}$ is accepted as reasonable (from experience at the sleep laboratory at Sir Charles Gairdner Hospital). The optical ranging distance range of the probe needs to be large enough that human airways can be completely measured. The probe needs to be sensitive to weak levels of light because, for optical ranging purposes, the airway wall is an "uncooperative target", that is, it is not an object that has been specifically designed to back-reflect the light from a particular probe. A brief overview of optical ranging, the various existing technologies and their applicability to the present application is now presented.

2.4.1 Optical ranging The use of light to measure the distance from an instrument to a light reflector or scatterer can be called optical ranging. While ranging, originally a military term, is understood to be measurement of the distance (or range) to a target, in this discussion the broader field of optical reflectometry is considered, that is, measurement of spatially-localized optical scattering. This is pertinent for two reasons, firstly because biological samples usually allow light to penetrate some distance below the surface and thus act as a collection of scatterers in space rather than as a single plane reflection such as a mirror. Secondly, the transparent catheter in which the probe is housed, and the probe optics themselves, will have a detectable amount of back-reflection, and this should not prevent the system from detecting the air-tissue interface.

Optical ranging has been used in a wide variety of scientific, industrial, mining, meteorological, telecommunications, commercial, and medical applications. Typically, the single-source, single-detector optical ranging methods can be clas- 
sified into pulsed time of flight, continuous wave modulation and coherence domain methods.

2.4.2 Time-of-flight ranging Time-of-flight methods operate by sending a pulse of optical energy, of high peak power but short temporal width, and then measuring the time delay for the reflection to return. They have a distance resolution limited by the speed of the photodetectors and electronic circuits; creation of the picosecond-level electronic circuits required to achieve resolution on the order of a millimetre would be technically challenging. An example of this type of distance ranging is optical time domain reflectometry (OTDR), used for characterizing the reflections in an optical fibre circuit. OTDR instruments achieve resolutions typically on the order of several millimetres. Schemes achieving millimetre resolution have required very high peak power levels (undesirable for in vivo application) and long averaging times - these requirements make OTDR unsuitable for this application.

LIDAR (LIght Detection And Ranging) is a term which has been used to describe measurement of optical backscattering over long distances in air. In a military context LIDAR is also known by the name LADAR (LAser Detection And Ranging). To measure distance usually time-of-flight methods are used; large pulses of optical energy is launched in the atmosphere and backscattering from dielectric discontinuities (both large object and small particles) is collected by a sensitive detector. These techniques also do not have the spatial resolution required for airway scanning application.

\subsubsection{Structured light and other non-interferometric methods Some rang-} ing methods employ structured illumination (e.g. the projection of grid lines), or multiple spatially separated detectors and/or light sources. These techniques, while very successful in mapping three-dimensional structures, are not incorporated easily into a catheter-mounted probe capable of nasal insertion due to the size and complexity of the optics required at the point of scanning. One such method is laser triangulation, for example, the handheld 3D laser scanner developed for movie costume design (Applied Research Associates NZ, New Zealand) and scanners for mapping works of art such as sculptures [14]. A miniaturized, endoscopic device using such a method has been created, but the endoscope was $5.1 \mathrm{~mm}$ in diameter and rigid [44], therefore unsuited to the nasopharyngeal airway. Laser triangulation requires a large separation between detectors for high resolution and unambiguous detection. Without this large separation, as in the 
case of endoscopic implementations, the technique does not accurately measure highly contoured and textured surfaces.

Laser diode feedback has been used as a mechanism for measuring range. A laser diode is used to illuminate a target, and some of the reflected light re-enters the laser cavity. This results in a disturbance to the laser operation as effectivly an external cavity has been created. One reported system had a range of $0.9-1.1 \mathrm{~m}$ with a resolution of $0.4 \mu \mathrm{m}$ [45], however the laser feedback system is only suitable for a feedback level over a preset range. The sample reflectance level in the $a \mathrm{OCT}$ system will experience large fluctuations due to the variations in tissue surface, angle of incidence and any moisture coating (mucous). The reported working range of $0.9-1.1 \mathrm{~m}$ would limit the probe length to approximately $1 \mathrm{~m}$, however $1.5-2.0 \mathrm{~m}$ is the preferred probe length for this application. Similar systems reported had ranges of $0.25-2.35 \mathrm{~m}$ with an accuracy of $2 \mathrm{~cm} \mathrm{[46],} \mathrm{and}$ $0.5-2.0 \mathrm{~m}$ with resolution $1.5 \mathrm{~mm}$ [47], both insufficient resolution. Laser diode feedback ranging systems have difficulty coping with the presence of multiple reflections, as would be the case with an optical probe contained in a plastic catheter.

There have been reports of non-interferometric continuous wave modulation methods, which are based on frequency modulation (FM) of a sub-carrier waveform. The distance ranging resolution of these methods is dependent on the maximum speed of the electronics (a very wide frequency sweep modulating signal must be generated) and typically they do not have sufficient resolution for this application. Published resolution values include $5 \mathrm{~cm}[48,49]$ and $0.25 \mathrm{~m}$ [50]. A significant problem with these incoherent FMCW techniques is that they have problems with detecting weak signals and separating multiple signals, particularly in the presence of stronger, unwanted reflections over the axial scan range.

2.4.4 Interferometric methods Interferometric techniques are those which use the coherence properties of the optical field to acquire information about the reflected light. These techniques require a light field to be split into two paths, one reflecting from the sample and the other a reference path, which are subsequently recombined at a detector. The resulting interference between the two optical fields is used to determine information about the sample such as distanceresolved reflectance.

As reported by Michelson and Benoit in 1895, the single-wavelength Michelson interferometer has been used since the invention of interferometers to detect the position or motion of a reflecting surface (eg. a mirror) by using the inter- 
ference signal to measure the phase difference between the sample and reference fields [51]. For strong, single-surface reflections, the method has position sensitivity much better than the wavelength of the light used. However, from measurements at a single point it is not possible to determine the position of a sample absolutely, as the measured phase repeats at distance intervals equal to the wavelength. Without modification the technique is unsuitable for absolute distance measurement.

The unambiguous range of a single-wavelength interferometry system can be extended with the use of two (or more) narrow linewidth sources, which must have well stabilised centre wavelengths to maintain the accuracy of the distance measurement. Michelson and Benoit were also aware of this technique in 1895, but the invention of the laser in 1960 provided the stabilised, narrow linewidth sources which were required [52]. There is a trade-off between the unambiguous range and resolution of a two-wavelength approach, which is controlled by the frequency spacing between the two wavelengths. There are a number of possible implementations of multiple-discrete-wavelength schemes, but to our knowledge none so far have been shown to be successful in the regime of fast acquisition times and weak, distributed signals (e.g. diffuse scattering from biological materials).

2.4.5 Optical coherence tomography An extension from laser interferometry using multiple discrete wavelengths is to use a source emitting light in a continuous range of wavelengths. All of the optical wavelengths at the interferometer output are in phase with each other and produce a characteristic intereference pattern only when the optical length of both interferometer paths are closely matched. The distance range over which the interferometer paths need to be matched to produce the interference pattern is inversely related to the source optical bandwidth. The technique has been described as low coherence interferometry (LCI), white light interferometry (WLI), or optical coherence-domain reflectometry (OCDR), and was initially used in the examination of fibre-optic components and circuits [53-55]. The technique is typically used with a distance resolution from the level of several micrometres up to several tens of micrometres, depending on the optical bandwidth of the source. The technique has good sensitivity to very weak reflections, as it is effectively an heterodyne technique where the strong local oscillator (the reference optical field) is used to detect a weak sample signal. With the addition of scanning optics to probe a sample in two dimensions the technique has been named optical coherence tomography 
(OCT) [56] and is now used in many biomedical applications; opthlamology [57], vascular medicine [58] and dermatology [59] are some of the most advanced. OCT typically has been used to capture tomographic images of biological structures in which light can penetrate, with a typical penetration depth of several millimetres or less in highly scattering tissues (skin, muscle, etc.).A brief introduction to the theory of OCT signal generation is given in Appendix D.

2.4.6 Endoscopic optical coherence tomography It is possible to implement an endoscopic sample arm in the OCT interferometer. The ability to use a thin endoscopic probe, which allows the examination of the microscopic structure and function of tissue, has the potential to reduce the need for more invasive biopsy and exploratory surgery. There is a trend towards the greater use of laparoscopic surgery, also known as keyhole surgery, because the surgery is less traumatic for the patient and it significantly improves recovery times. Endoscopic tools with new capabilities could help in this.

Medical uses of endoscopic OCT to date include applications in the gastrointestinal tract from the oesophagus [60-63] and stomach [61] to the intestine [64, 65], bladder [66,67], the larynx [68], gynaecological applications [67], and bronchial airways [69]. Imaging tissue on a microscopic level to examine lesions and possible cancerous tissue has been a strong area of research in OCT and the motivation for most of these reported systems. The systems have used a variety of scanning modalities. These include rotation of a perpendicularly-directed beam [58, 70], linearly scanning a perpendicular beam back and forth inside along a catheter [71], and forward-looking endoscopic probes [72].

Endoscopic OCT has been shown to be effective for examining the lumen of blood vessels as a replacement technology for intravascular ultrasound (IVUS) $[58,70]$. It has been possible to image the structure of artery walls, to measure the thickness of various layers and identify the likely composition of deposits in the vessel walls. Figure 2.6 shows an example of imaging a stent in a blood vessel, to check the success of deployment and investigate long term effects.

For OCT applications the typical reported scan range has been a few millimetres. This is primarily because the penetration depth of most highly scattering tissue types is approximately one or two millimetres. In contrast, the size of the airway can be greater than $50 \mathrm{~mm}$ in diameter at certain points, requiring a much greater scan range. The successful extension of the OCT scan range to achieve measurement of this structure is discussed in Chapter 3. The typical reported distance resolution of OCT is from 5 to $20 \mu \mathrm{m}$, depending on the characteristics of 

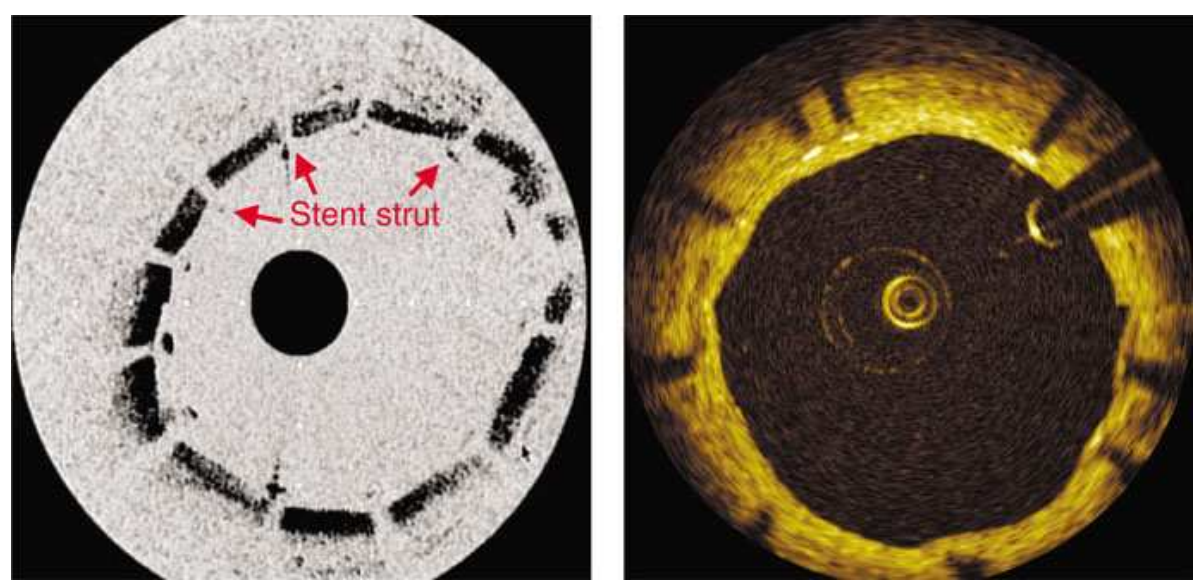

Figure 2.6: In vivo intravascular OCT images of (left) rabbit aorta and (right) human coronary artery showing stents in place. During image acquisition the blood was temporarily replaced with a saline flush. The blood vessels are several millimetres in diameter. (Source: [73])

the light source. This is more than adequate for the use in airway structure measurement. For comparison, the typical resolution of CT and MRI machines is 0.5 to $1.0 \mathrm{~mm}$. As stated above, a $1 \mathrm{~Hz}$ frame rate or greater is required for the $a \mathrm{OCT}$ probe. The reported rates of various endoscopic OCT system have surpassed this - up to $4 \mathrm{~Hz}$ frame rate [58]. Therefore, endoscopic OCT is suitable in terms of catheter size, resolution, and frame rate.

\subsection{Summary and conclusions}

This chapter has presented the motivation for a new instrument to dynamically measure airway shape over extended periods and during sleep. In recent years sleep apnoea has been recognized as a potentially serious condition with high social and economic costs. The main treatment options and the problem of low success rates of treatments other than CPAP were described. The current airway size and shape measurement techniques were discussed, as well as the potential for a new instrument to improve understanding of OSA, allow better treatment selection and possibly the development of new treatments. The concept of the instrument based on an optical probe in a naso-oesophageal catheter was presented. Finally, the optical technology employed in the instrument, endoscopic optical coherence tomography, was briefly introduced. 


\section{Key OCT design issues}

The purpose of this chapter is to describe two key OCT design issues in the aOCT system, namely the design of the long-range delay line and the OCT sensitivity.

Typically, most reported OCT systems have had a depth range of several millimetres and an optical delay line with a scanning range to match. Our delay range requirement is much longer in order to measure the larger distances encountered in the human upper airway. A survey of existing delay line designs was carried out and the frequency-domain optical delay line (FDODL) was chosen. In order to achieve a range from the FDODL suitable for this application, we used optical ray-tracing to investigate the important parameters and limitations on the range and performance. The raytracing was done with Ian D. Walton who was on a sabbatical visit at OBEL. This work was published in an Optics Express article [74].

The $a \mathrm{OCT}$ probe has a low numerical aperture as it is contained within a small package and is designed to achieve a consistent response over an extended range. Consequently, it collects very little backscattered light and therefore achieving high sensitivity from the OCT system is important. The section on OCT sensitivity is partly based on work in a published book chapter by Sampson and Hillman [75] to which I contributed.

\subsection{Delay line function and requirements}

The function of the scanning optical delay line in a time-domain OCT system is to impart a variable time delay to a light wave. The scanning speed and repetition rate required of the delay line depends on the type of scanning used. Typically, in a time-domain OCT system a focused beam is scanned through the sample in two or three dimensions to build up the data set, and the scanning priority is as follows: Firstly, the beam is scanned into the sample, the z-direction, to capture a reflectivity profile which is called an "A-scan" (ultrasound scanning terminology). At a slower speed, x-scanning (and possibly y-scanning) optics scan the beam laterally across the sample. In this scheme the optical delay scanning is the fastest optical scanning in the system.

The simplest form of scanning delay line is a mirror oriented perpendicularly to a light beam. The mirror can be translated back and forth along the direction of the beam to change the path length that the beam travels. However, the scanning speed is slow and even with the use of a lightweight mirror mounted on a PZT 
actuator the inertia of the mirror limits its speed to a level where scanning of the tissue of living animals becomes impossible due to the image-degrading motion artifacts.

The required distance range and repetition were two key parameters of the OCT system which guided the selection of delay line technology.

Range: The delay line scan range of the aOCT system was planned based upon the typical dimensions of the upper airway, and also some consideration of component availability and equipment size. Ciscar et al. used MRI to examine the dimensions of the velopharynx, a typical site of airway collapse, in a study of 8 healthy and 17 sleep apneic middle-aged adults [76]. The maximum values of their dimension measurements in the velopharynx are shown in Table 3.1. The study found that apnoea sufferers had more variability in their airway size during the breathing cycle, but that the variation was usually towards a smaller size.

\begin{tabular}{|l|l|l|}
\hline Dimension & Normal & Apneic \\
\hline Lateral $(\mathrm{mm})$ & $16.4 \pm 2.3(13-21)$ & $10.6 \pm 5.5(2-25)$ \\
AP $(\mathrm{mm})$ & $8.7 \pm 3.2(6-11)$ & $8.2 \pm 2.4(5-15)$ \\
CSA $\left(\mathrm{mm}^{2}\right)$ & $121.9 \pm 7.9(83-113)$ & $85.9 \pm 49.9(19-173)$ \\
\hline
\end{tabular}

Table 3.1: Maximum velopharyngeal airway dimensions measured by Ciscar et al. AP and CSA are the anterposterior distance and crosssectional area. The values given are mean \pm standard deviation (minimum - maximum).

Trudo et al. used MRI to examine the airway dimensions in the retropalatal, retroglossal and epiglottal pharyngeal regions in a study of 15 normal subjects [77]. Figure 3.1 shows a bar graph of the mean and standard deviation of the anteroposterior (AP) and lateral dimensions in the three regions, for sleep and awake states. The plot shows that the mean maximum diameter of the airway in the lateral and AP directions occurs in the epiglottis during wakefulness in the lateral direction, and was approximately $25 \mathrm{~mm}$. The standard deviation of the lateral dimension at this site was $6.8 \mathrm{~mm}$.

Taking this information and discussions with airway physiologists who collaborated in the research ${ }^{1}$ into account, the first stage target radial range was $12.5 \mathrm{~mm}$ (25 mm diameter) and the second stage target was $25 \mathrm{~mm}$ (50 mm diameter).

Repetition rate: The required delay-line repetition rate, $f_{r e p}$, is determined by

\footnotetext{
${ }^{1}$ Dept. Pulmonary Physiology, Sir Charles Gairdner Hospital
} 


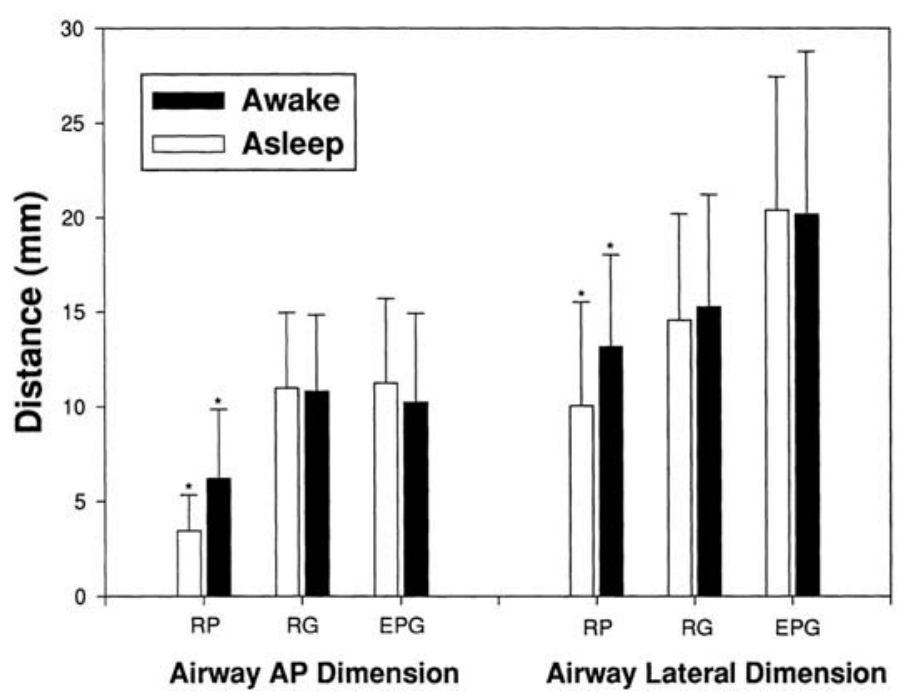

Figure 3.1: Bar graph of retropalatal (RP), retroglossal (RG) and epiglottal (EPG) pharyngeal measurements during sleep and wakefulness from Trudo et al.

the required angular resolution, $\Delta \theta$, and the rotation rate of the probe, $f_{\text {rot }}$;

$$
\Delta \theta=\frac{2 \pi f_{\text {rep }}}{f_{\text {rot }}}
$$

$f_{\text {rot }}$ must be high enough that motion of the airway does not disturb the images significantly, as discussed in Chapter 2. The initial goal of 1 rotation per second and 250 radial scans per revolution $\left(\Delta \theta=1.4^{\circ}\right)$, gives a required $f_{\text {rep }}$ of $250 \mathrm{~Hz}$.

\subsection{The FDODL}

To achieve images free of motion artefacts the use of scanning speeds higher than translating mirrors can achieve is required, on the order of several hundred Hertz or more. From the mid-1990s to the present day a variety of different schemes developed to accomplish this have been published, but the one most commonly employed in OCT imaging systems has been the frequency-domain optical delay line (FDODL), also known as the rapid scanning optical delay line (RSOD). The FDODL consists of a combination of grating, lens, and tilting mirror to achieve a variable optical delay. The origin of the FDODL is in the field of ultra-short pulse shaping $[78,79]$. It was first used to generate a scanning delay by Kwong [80], and consisted of a grating, lens and PZT-driven tilting mirror. It was also shown that, in addition to scanning the group delay, the FDODL could be used to add a phase ramp in the wavelength domain of the light field. The first use of the FDODL in OCT was reported by Tearney [70], with the mirror driven 
by a galvanometer to expand the scanning range. This meant that the fringe frequency of the interference signal from the OCT system could be easily controlled by setting the position of the galvanometer pivot. Subsequently Rollins [81] published the addition of a double-pass mirror which doubled the delay range and made optical alignment more convenient. The FDODL has been used in many later reported OCT systems. Scan ranges and repetition rates demonstrated by some of the published systems are shown in Table 3.2. Of note is the very long range form of the FDODL first reported by Silva [82], where a polarization beam splitter and quarter-wave plate were used to allow the FDODL to be used over a longer range. This will be described in more detail below.

A variation in the use of the FDODL was the 'adaptive ranging' use reported by Iftimia et al. [83]. In the system they described, in any single A-scan the FDODL was scanned only over part of its full range. While the depth range of the A-scan was kept constant, the offset of the A-scan was dynamically changed so that only the region-of-interest in the sample was imaged. The advantages of this scheme are that fewer points need to be sampled, and consequently the bandwidth of detection can be reduced, thus increasing the system sensitivity. The major disadvantage of this technique is that the offset of each A-scan must be determined before the scan is performed. This is usually done by looking at the previous image to determine where the region-of-interest was and making any slight adjustments if necessary. However, this method is not capable of handling a sudden change in the sample geometry, such as when new features become present in the image, or the sample surface moves rapidly. Both of these situations are expected to be commonplace in aOCT imaging, thus making the adaptive ranging use of the FDODL unsuitable in this application.

3.2.1 Description of FDODL operation The operation and theory of the FDODL has been well covered previously [87-89], although a simplified explanation is useful here to introduce the features of interest which are examined in the modeling section below. Figure 3.2 illustrates the mechanism by which the FDODL generates an optical delay which is controllable by the rotation of a galvanometer mirror. Briefly, the analysis of the wavelength-dependent phase delay imparted to the beam shows that, for small galvanometer tilt angles $(\sin \theta \approx \theta$ ), the group delay is linearly proportional to the galvanometer angle. The derivative of phase delay with respect to tilt angle is linearly proportional to the offset of the galvanometer pivot from the lens axis (the direction of offset is in the lens focal plane and perpendicular to the galvanometer axis). Effectively, a Doppler 


\begin{tabular}{|c|c|c|c|c|}
\hline Year & Publication & $\begin{array}{l}\text { Usable scan } \\
\text { depth }\end{array}$ & $\begin{array}{l}\text { Scan repeti- } \\
\text { tion rate }\end{array}$ & comment \\
\hline 1993 & Kwong [80] & $0.6 \mathrm{~mm}$ & $400 \mathrm{~Hz}$ & \\
\hline 1997 & Tearney [70] & $3 \mathrm{~mm}$ & $2 \mathrm{kHz}$ & $\begin{array}{l}\text { resonant op- } \\
\text { eration }\end{array}$ \\
\hline 1998 & Rollins [81] & $2 \mathrm{~mm}$ & $4 \mathrm{kHz}$ & $\begin{array}{l}\text { resonant op- } \\
\text { eration }\end{array}$ \\
\hline 1999 & Silva [82] & $23 \mathrm{~mm}$ & $30 \mathrm{~Hz}$ & \\
\hline 2000 & Zhao [84] & $4 \mathrm{~mm}$ & $400 \mathrm{~Hz}$ & \\
\hline 2001 & de Boer [85] & $2.8 \mathrm{~mm}$ & $1 \mathrm{kHz}$ & \\
\hline 2002 & Rollins [86] & $5 \mathrm{~mm}$ & $8 \mathrm{kHz}$ & $\begin{array}{l}\text { resonant op- } \\
\text { eration }\end{array}$ \\
\hline
\end{tabular}

Table 3.2: Parameters of reported frequency-domain optical delay lines.

frequency shift of controllable magnitude and sign is applied to the light by the delay line when it is scanning. This has the advantage that the frequency of the interference fringes can be freely chosen and the detection bandwidth can be set to a frequency range where the electronic detection circuit has a low noise level. The fringe frequency is usually chosen to be high enough that the frequency content of the envelope and fringes are well separated, allowing efficient envelope detection.
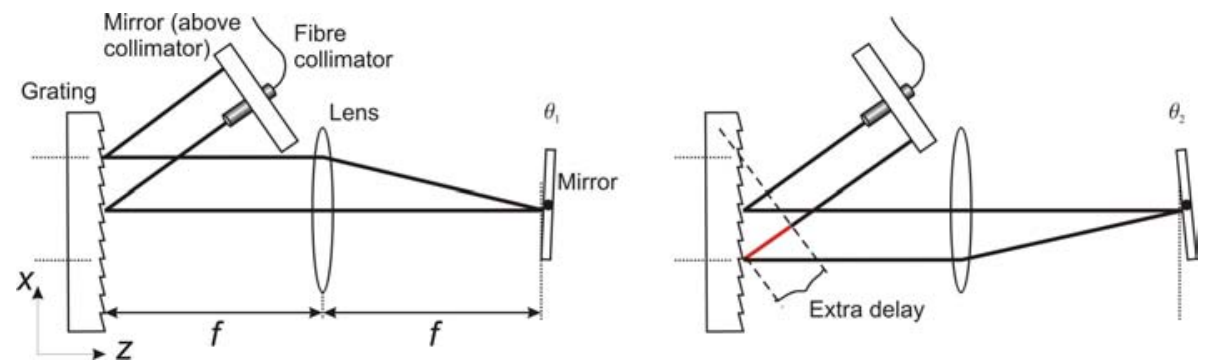

Figure 3.2: The FDODL operates by tilting the galvanometer mirror. The light field group delay is proportional to the galvanometer angle, $\theta$. The Doppler shift can be controlled by setting the offset of the galvanometer pivot.

3.2.2 Other delay lines OCT systems using delay lines technologies other than the FDODL have been surveyed and a selection of the designs are summarized in Table 3.3. Most of these are unsuitable for use in the $a$ OCT system for reasons of insufficient range or repetition rates, or difficulties concerning parameter flexibility, system complexity or ease of alignment. Those utilizing large rotating glass components suffer from variable dispersion across the scan. De- 
lay range extension by delaying the light multiple times with a recirculating fibre loop has been demonstrated [90]. A modulating element can be placed in the loop to frequency-multiplex the signals to distinguish light which has experienced different numbers of loop passes. The disadvantages of this technique are the increased complexity and the reduced system sensitivity due to the division of the reference arm optical power into the multiple passes.

\subsection{The impact of FDODL configuration on achieving a long range scan}

The basic operation of the FDODL has been described above. The delay line scan range is defined as the distance over which the FDODL delay can be scanned while maintaining an optical power level greater than $50 \%$ of the maximum power level. The particular configuration used affects the maximum possible scanning range. Variations in the FDODL configuration which have been examined to determine their impact on the scanning range are explained below.

In-plane and out-of-plane: The first variation is illustrated in Figure 3.3, a setup in which the beams in the FDODL all lie in a single plane which passes through the lens centre. We use the term "in-plane" to describe this design choice. Due to the relative positions of the collimator and double-pass mirror, for zero galvanometer angle the beam returns to the collimator after a single pass rather than reflecting from the double-pass mirror. Then the full aperture of the optical components cannot be accessed and the range is truncated; therefore it is only suitable for short range scanning.

(a)

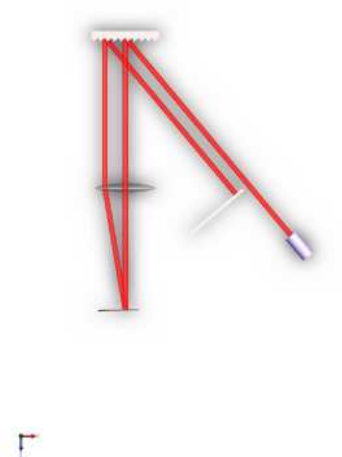

(b)

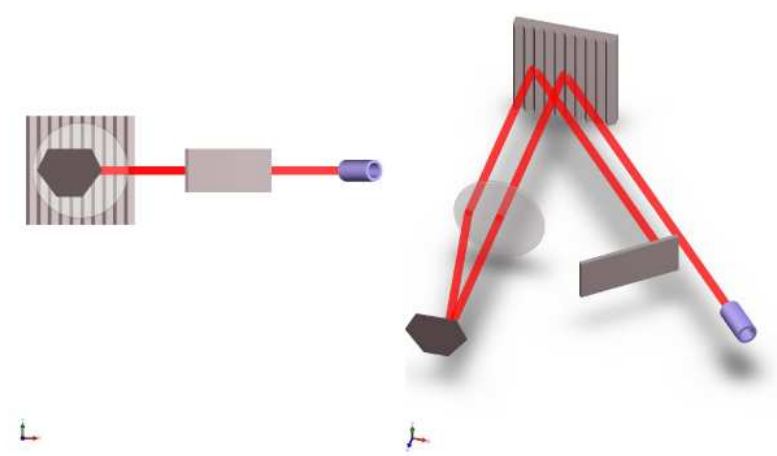

Figure 3.3: In-plane FDODL configuration. The collimator and doublepass mirror prevents the beam from being scanned across the full aperture of the optical components. (a) Plan view, (b) front elevation, (c) perspective. For clarity the dispersion of the beams by the grating is not shown. 


\begin{tabular}{|c|c|c|c|c|}
\hline Year & Publication & Delay line type & Scan depth & Scan repetition rate \\
\hline 1992 & Swanson [91] & $\begin{array}{l}\text { Retroreflector on gal- } \\
\text { vanometer }\end{array}$ & $20 \mathrm{~mm}$ & $1.9 \mathrm{~Hz}$ \\
\hline 1996 & Tearney [92] & PZM fibre stretcher & $2.2 \mathrm{~mm}$ & $1.2 \mathrm{kHz}$ \\
\hline 1997 & $\mathrm{Su}[93]$ & rotating cube & $1.5 \mathrm{~mm}$ & $22 \mathrm{~Hz}$ \\
\hline 1997 & Ballif [94] & rotating cube & $50 \mathrm{~mm}$ & $384 \mathrm{~Hz}$ \\
\hline 1997 & Delachenal [95] & $\begin{array}{l}\text { rotating partially- } \\
\text { gold-coated cube }\end{array}$ & $118 \mathrm{~mm}$ & $76.25 \mathrm{~Hz}$ \\
\hline 1998 & Szydlo [96] & $\begin{array}{l}\text { air-turbine-driven ro- } \\
\text { tating cube }\end{array}$ & $2 \mathrm{~mm}$ & $28.6 \mathrm{kHz}$ \\
\hline 1999 & Giniūnas [97] & $\begin{array}{l}\text { rotating parallelo- } \\
\text { gram prism }\end{array}$ & $6.9 \mathrm{~mm}$ & $50 \mathrm{~Hz}$ \\
\hline 2001 & Lai [98] & rotating prism array & $3.5 \mathrm{~mm}$ & $2 \mathrm{kHz}$ \\
\hline 2002 & Chen [99] & $\begin{array}{l}\text { mirrors on rotating } \\
\text { disk }\end{array}$ & $2 \mathrm{~mm}$ & $2.4 \mathrm{kHz}$ \\
\hline 2003 & Shiina [100] & $\begin{array}{l}\text { retro-reflectors on a } \\
\text { rotating disk }\end{array}$ & $40 \mathrm{~mm}$ & $15 \mathrm{~Hz}$ \\
\hline 2004 & Liu [101] & $\begin{array}{l}2 \text { flat (one rotating) } \\
\text { mirrors, } 1 \text { curved mir- } \\
\text { ror }\end{array}$ & $1.5 \mathrm{~mm}$ & $4.1 \mathrm{kHz}$ \\
\hline
\end{tabular}

Table 3.3: A survey of other delay line designs. 
A schematic and 3D rendered model of a long range FDODL using a polarization beam splitter (PBS), first described by Silva [82], is shown in Figure 3.4. Light initially entering the FDODL has the correct linear polarization state so that it will pass through the PBS without reflecting. It then passes through a quarter-wave plate with the crystal axis aligned at 45 degrees to the polarization direction. This changes the polarization state to circular. The light reflects from the grating-lensmirror section, passes the quarter-wave plate again and is converted back to a linear polarization, though orthogonal to the original input light. The light encounters the PBS and reflects this time to a double-pass mirror, which is aligned so that the light beam will reflected back along the same path. The purpose of the polarization components in this FDODL is to allow the light beam in the FDODL to remain in one plane throughout. Therefore, it is also an in-plane configuration, but one that avoids the crowding of the collimator and double-pass mirror. The maximum possible range is then limited by the apertures of the lens, grating and double-pass mirror, and is given by

$$
L_{\text {range }}=A \lambda_{0} / p
$$

where $\lambda_{0}$ is the centre wavelength of the broadband source, $A$ is the available aperture, and $p$ is the grating pitch. This equation shows that the delay line range can be increased by increasing the aperture of the components. To achieve a long scan range in this way it is straightforward to obtain gratings and mirrors of larger aperture; however, increasing the lens aperture without changing its focal length would result in an impracticably large or physically impossible numerical aperture. Instead, the focal length should be increased by the same factor as the aperture, which maintains the same NA. The result of this is that the grating-lens distance and the lens-galvanometer-mirror distance, which are both approximately equal to the lens focal length, will be larger, and therefore the entire delay line will be physically larger. The larger size may have an impact on the alignment stability and the practical considerations of engineering a portable OCT system.

The "out-of-plane" FDODL configuration is shown in Figure 3.5. The beams incident on the grating and passing through the lens are parallel but not coplanar, and the double-pass mirror is placed at a level below the collimator. This choice of configuration has been illustrated in the system diagrams of some reported OCT systems [102-104]. To achieve a maximum scanning range, the beam which is 
(a)

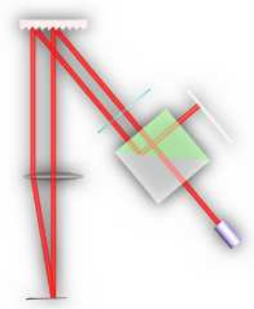

$\Gamma$ (b)

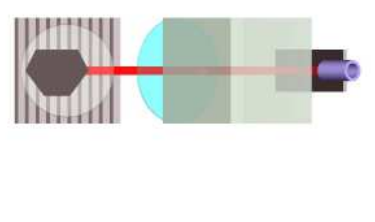

L (c)

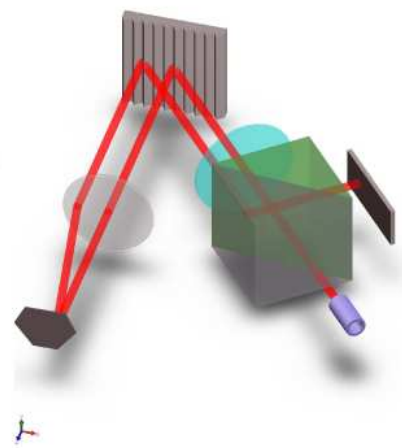

Figure 3.4: In-plane PBS-based FDODL configuration. The collimator prevents the beam from being scanned across the full aperture of the optical components. (a) Plan view, (b) front elevation, (c) perspective.

scanned across the lens by the galvanometer mirror should be located in a plane which passes through or near the centre of the lens. This ensures that the full aperture of the lens is used.

(a)

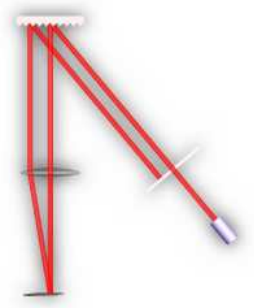

$\Gamma$ (b)

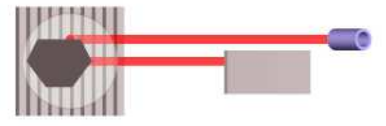

(c)

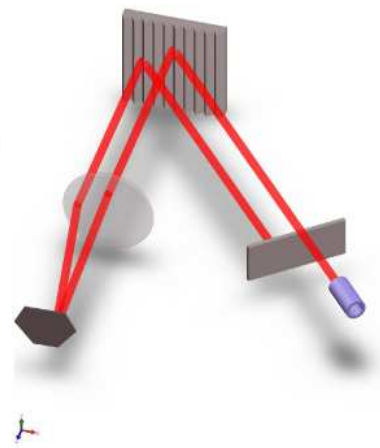

Figure 3.5: Out-of-plane FDODL configuration. The non-coplanar alignment allows the beam to be scanned across the full aperture of the optical components.

"On-pivot" or "off-pivot": Figure 3.6 illustrates the on-pivot and off-pivot configurations. As mentioned above, the position of the galvanometer pivot has an effect on the Doppler shift of the reference field. The use of the on-pivot configuration means that the interferogram fringe frequency will be zero.

3.3.1 Description of modelling procedure ZEMAX (ZEMAX Development Corporation, Bellevue, Washington, USA) was used for optical modelling of the 
(a)

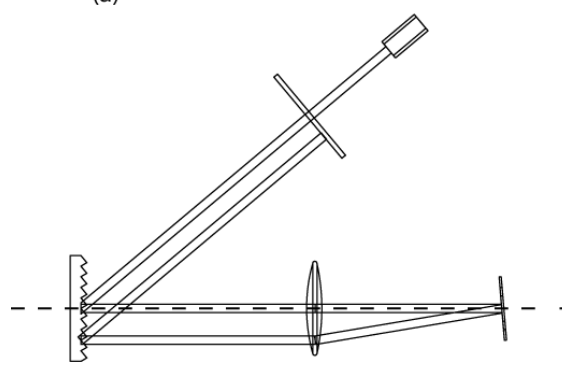

(b)

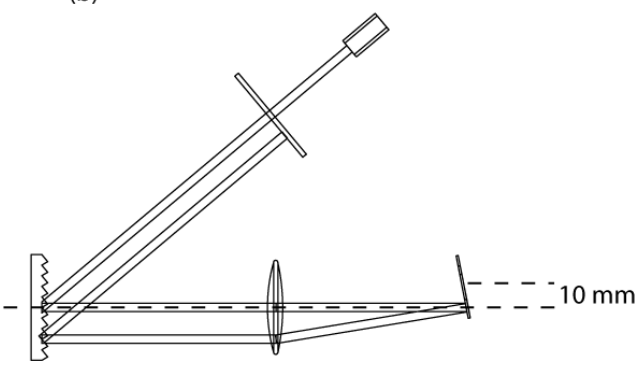

Figure 3.6: (a) "on-pivot" and (b) "off-pivot" FDODL configurations

FDODL scan range ${ }^{2}$. The model consisted of an arrangement of single-mode fiber, collimator (ZEMAX lens prescription for the Shäfter+Kirchhoff M20 collimator lens), grating (400 lines per mm), achromatic doublet lens (190-mm focal length, ZEMAX lens prescription for the Melles Griot 06-LAI-015), and mirror, see Figure 3.7, with delay line dimensions that closely match the experimental FDODL which was built. Using the model we examined the effect of using an in-plane or out-of-plane configuration. For the out-of-plane configuration, the input beam and beam reflected from the galvanometer mirror are separated by $20 \%$ of the lens aperture and are equidistant above and below the optical axis. We also examined the effect of placing the pivot point of the galvanometer mirror either on or $10 \mathrm{~mm}$ away from the focal point but still in the focal plane, i.e., either on-pivot or off-pivot. The offset was chosen to provide the minimum phase modulation necessary to avoid the problem of phase fading, i.e., slightly in excess of one fringe per envelope. This choice represents the best case for the off-pivot configuration. The fringe frequency is proportional to the pivot offset [70]. For better envelope detection a greater offset would usually be used to achieve perhaps 5-10 fringes per envelope. Three equally spaced wavelengths were used in the model, weighted according to a Gaussian spectrum centered on $1325 \mathrm{~nm}$ with a width (FWHM) of $54 \mathrm{~nm}$. The model calculations included all aberrations and vignetting, and used the built-in ZEMAX fiber-coupling model, which is a hybrid overlap-integral approach following Wagner and Tomlinson [105]. The optical fibres for launch and collection had parameters based on Corning SMF28 fibre and had the same spatial location and orientation to model the physical FDODL where one optical fibre is used for both input and output. We modeled the four possible combinations of the in-plane or out-of-plane configuration, and

\footnotetext{
${ }^{2}$ The ZEMAX modelling was carried out in collaboration primarily with Ian D. Walton. Andrei V. Zvyagin and Sergey A. Alexandrov also assisted with early discussions and preliminary modelling which is not presented here.
} 
off-pivot or on-pivot settings. ZEMAX macros were written to step through the galvanometer angles and wavelengths and record the fibre coupling efficiency for each setting.
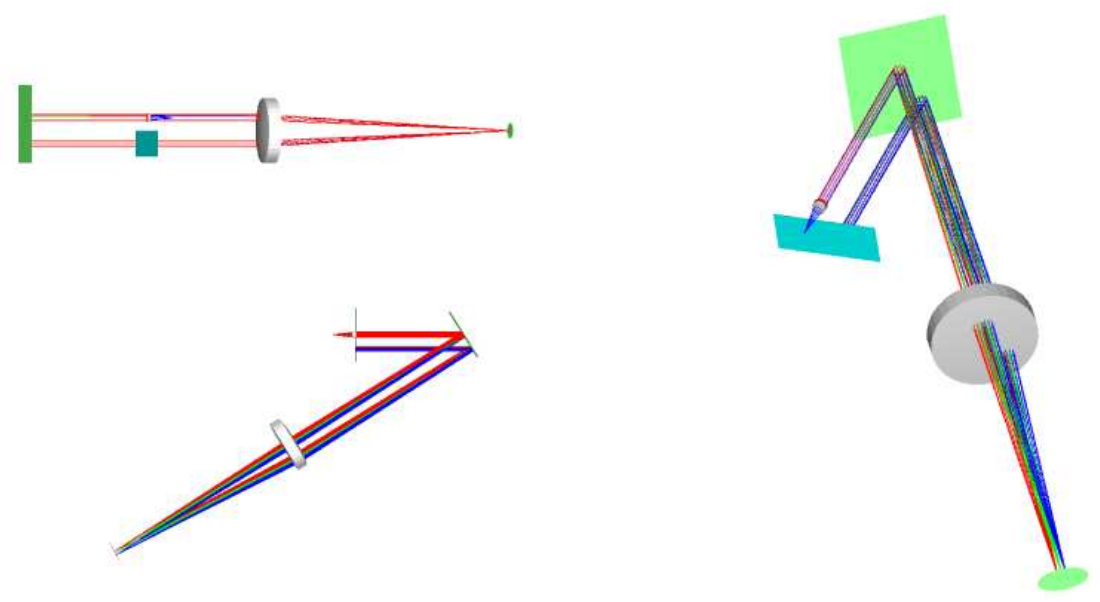

Figure 3.7: ZEMAX model views

3.3.2 Results Figure 3.8 shows the results of calculations of the coupling efficiency versus scan position, plus a measured coupling efficiency for an FDODL using the same parameters as above, configured with the beam on-axis and the focal point at the galvanometer pivot. Relative coupling efficiency is defined as the ratio between the input and output power of the delay line. There is good agreement between the measured and theoretical curves. The theoretical maximum range of the FDODL, determined by locating the mean wavelength at the lens edge, is given by $A \lambda_{0} / p$ (26.6 $\mathrm{mm}$ in this case), where $A$ is the available aperture (of lens, grating and mirror), $\lambda_{0}$ is the center wavelength, and $p$ is the grating pitch [82]. The plot in Figure 3.8 shows the importance of placing the galvanometer pivot at the lens focal point. The relative loss in this case is less than 1.5 $\mathrm{dB}$ over the predicted range for either in-plane or out-of-plane configuration (the curves are not shown separately on the plot because they overlap almost exactly). The maximum relative loss over the same range for the measured curve is $1.9 \mathrm{~dB}$. The results presented in Figure 3.8 are consistent with previous experimental results [82].

As a check on the alignment of the FDODL used for the experimental results, the optical spectrum of light reflected from the FDODL was recorded for a series of delay positions, see Figure 3.9. The plot shows that the spectrum is not dependent on the galvanometer mirror angle except at the extreme ends of the $26.6 \mathrm{~mm}$ 


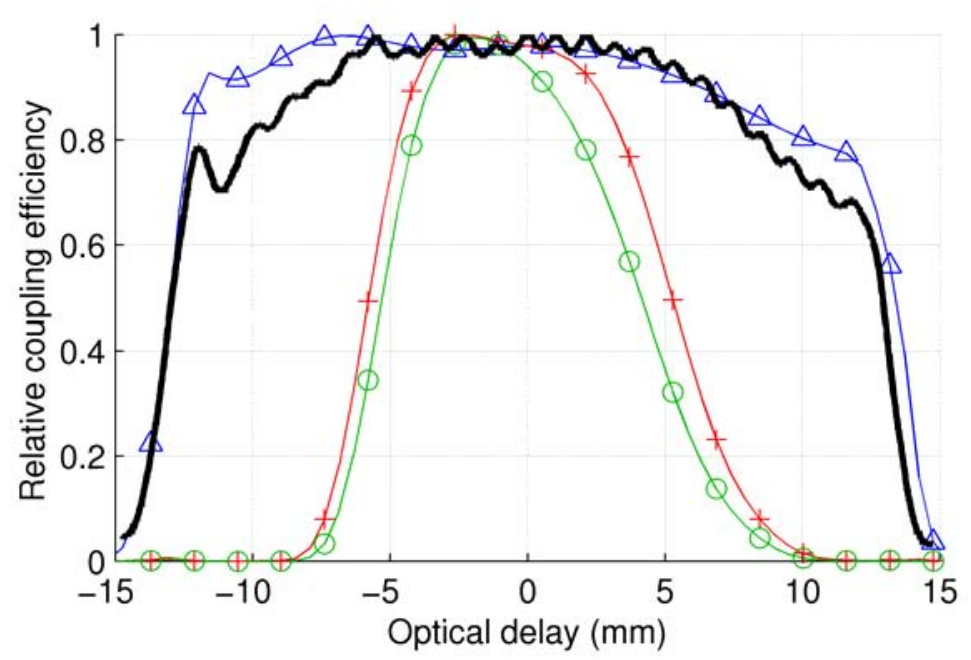

Figure 3.8: Results of the ZEMAX modelling (published in [74]). The relative coupling efficiency of the FDODL versus delay for the configurations: (o) off-axis and off-pivot, $(+)$ on-axis and off-pivot, and $(\Delta)$ both the off-axis and on-pivot, and on-axis and on-pivot. The heavy solid line is an experimentally measured response for the on-axis, onpivot configuration.

scan. The extreme edges of the scan are not used as the spectrum is strongly and unevenly attenuated and this would affect the resolution and sensitivity.

3.3.3 Discussion The modeling of the FDODL provides several insights into its performance. Firstly, the range for the off-pivot configurations is much worse than the on-pivot configurations because the beam focussed onto the galvanometer mirror is not reflected from the focal plane of the lens except at zero tilt angle, thus, it is not properly recollimated by the lens. This causes the return beam at the fiber to have a scan-dependent error in location and focus, which could be described as a "descan" error, and which causes a reduction in coupling efficiency. Secondly, comparing the in-plane and out-of-plane configurations, a slight degradation in the power efficiency of the off-axis configuration arises from increased aberration caused by the utilization of a larger fraction of the lens aperture. However, for a lens of long focal length, the NA is low and so we find that performance of the in-plane and out-of-plane configurations are very similar. Thirdly, after dispersion by the grating the different wavelengths of the beam pass through the lens at different lateral positions. Therefore, for a symmetric galvanometer scan the individual wavelengths are asymmetrically scanned across the lens. As a result, chromatic aberration of the lens causes an asymmetric curve of coupling effi- 


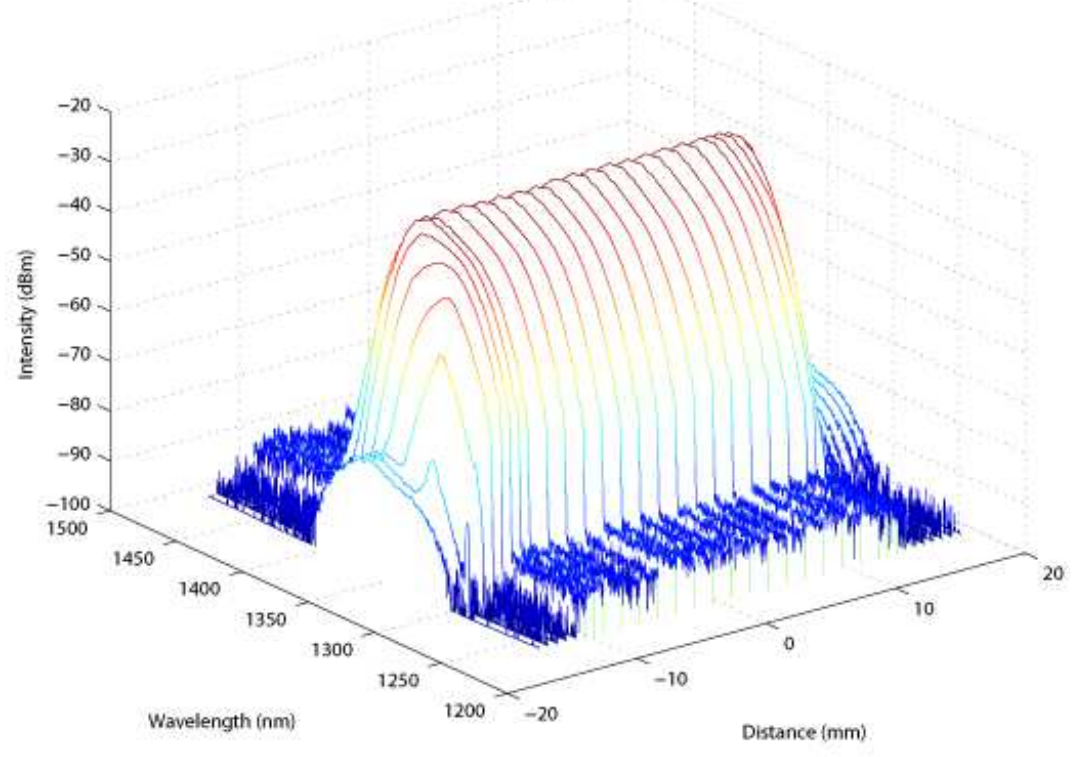

Figure 3.9: Output spectrum of the FDODL versus delay showing that spectral modulation is low except at the extreme edges of the scan.

ciency versus delay. These three effects cannot be eliminated completely, but their impact may be reduced somewhat by the use of a well-designed lens. All three effects become much more pronounced if a singlet lens is used in the FDODL.

There are other interesting areas that could be examined via the same type of modeling and experimental measurement of the FDODL response, in particular, the effect of the spectral filtering of the FDODL, and its variation versus delay scanning for different configurations. Such modeling would particularly apply if one were considering ultra-high resolution OCT with broad-spectrum sources. A similar ZEMAX model has been used to investigate the bandwidth limitations of FDODL configurations using achromatic lenses or aspherical mirrors in place of the lens [106].

\subsection{OCT sensitivity}

The $a \mathrm{OCT}$ system is not required to detect sub-surface tissue reflections, however, due to the low probe numerical aperture and long scanning distances, the required detectable signal level of the $a \mathrm{OCT}$ system is still very low - on the order of $-90 \mathrm{~dB}$ or less. Therefore it is important to design the system to maximise the OCT sensitivity, and this can be achieved by an understanding of the impact of the various interferometer parameters. 
Sensitivity definition The sensitivity of the OCT system is determined by the reflectivity of the sample which produces a signal-to-noise ratio (SNR) of unity. This is known as the minimum detectable reflectivity, $R_{\min }$, of the system and is the solution to the equation $\operatorname{SNR}\left(R_{\text {sample }}\right)=1$. The $\mathrm{SNR}$ is the ratio between the (electrical) signal power peak to the mean noise power (Figure 3.10). For convenience, sensitivity is defined as the inverse minimum detectable reflectivity, $S=R_{\mathrm{min}}^{-1}$. The sensitivity has also been described as the "system SNR" or "dynamic range" of the system [91,92]; then, it is implicitly understood that the value is the ratio from $100 \%$ reflection to $R_{\min }$. To measure the $R_{\min }$ of a system, we consider the case of a single point reflector which is part of a single A-scan.

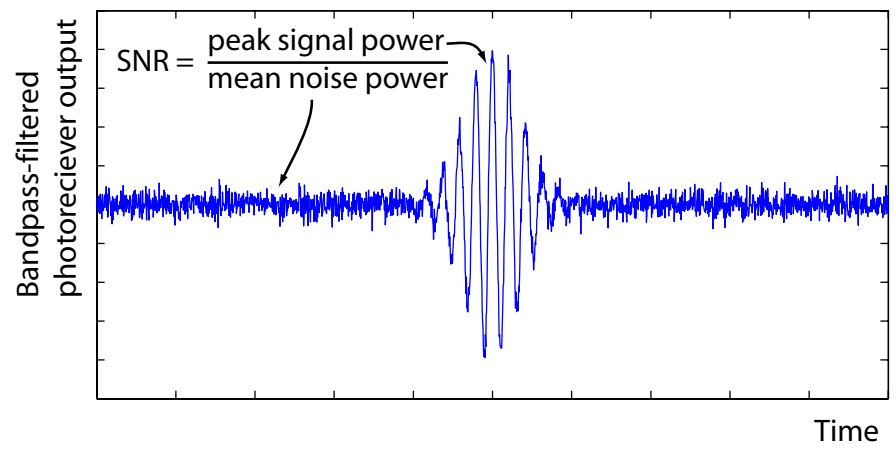

Figure 3.10: SNR definition.

Figure 3.11 shows the OCT system configurations which will be considered in this analysis.
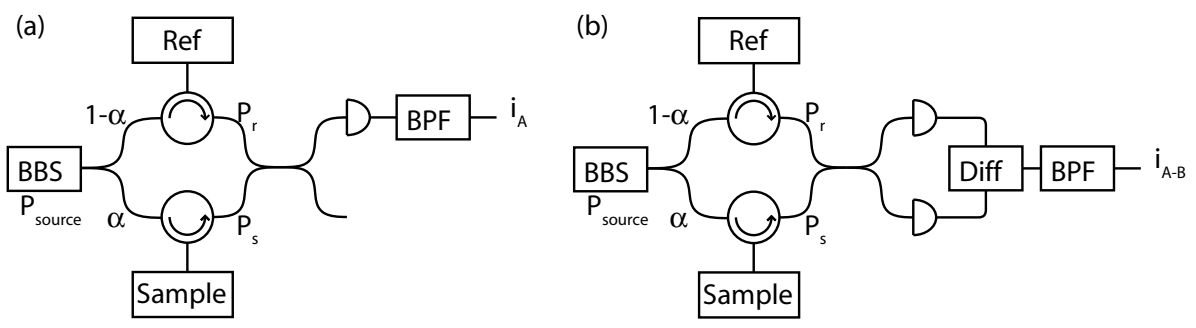

Figure 3.11: (a) Single-ended OCT interferometer and (b) dual balanced OCT interferometer. BBS, broadband source; BPF, bandpass filter; Diff, difference (subtraction).

3.4.1 Single-ended configuration We first consider the case of the sensitivity in a single-ended OCT system by considering the electrical signal in Figure 3.11(a), the bandpass-filtered photocurrent,

$$
i_{A}=\rho\left|u_{A}\right|^{2} \otimes h_{f}
$$


The light intensity at that photodetector is the square of the electric field amplitude (averaged over the photodetection response time). The total electric field $u_{A}$ is the combination of the electric field contributions from the reference and sample arm paths in the interferometer. Here the responsivity factor $\rho$ includes the quantum efficiency and photodetector area, and $h_{f}$ is the bandpass filter response.

Signal power The signal component of the OCT bandpass-filtered photocurrent in the close vicinity of a single reflector is given by

$$
i_{\text {signal }}\left(t-t_{0}\right)=2 \rho \sqrt{1 / 4 P_{s} P_{r}} \gamma(t) \cos \left(\omega_{f} t+\phi_{0}\right) .
$$

$\gamma(t)$ is the normalized $(\gamma(0)=1)$ cross-correlation function of the interfering reference arm and sample arm electric fields. $\omega_{f}$ is the OCT fringe frequency and $\phi_{0}$ is a static phase term. $P_{r}$ and $P_{s}$ are the optical powers of the light from the reference mirror and the sample reflector. They can be expressed in terms of the reference and sample reflectivities, $R_{r}$ and $R_{s}$;

$$
\begin{aligned}
& P_{r}=(1-\alpha) R_{r} P_{\text {source, }} \text { and } \\
& P_{s}=\alpha R_{s} P_{\text {source }} .
\end{aligned}
$$

where $\alpha$ is the coupler splitting ratio as shown in Figure 3.11, and then at $t=0$ we have the peak electrical signal power

$$
P_{\text {single, }, \text { signal }}=\frac{1}{2} \alpha(1-\alpha) \rho R_{s} R_{r} P_{\text {source }}^{2}
$$

where we have used the fact that $\overline{\cos ^{2}\left(2 \omega t+\phi_{0}\right)}=1 / 2$ and the assumption that the fringe frequency is greater than the envelope frequency content (i.e. $\omega_{f} \gg$ the bandwidth of $\gamma(t))$.

Noise power There are three fundamental noise sources which are taken into account in this analysis - shot noise, optical mixing noise, and thermal noise. These noise sources arise from independent physical processes and can be treated as uncorrelated signals; therefore it is appropriate to sum their mean powers [107]. Other noise sources such as electromagnetic interference, sampling jitter, quantization noise and amplifier noise also need to be considered carefully in the engineering design of a system. Those which have a flat frequency spectrum, independent of signal level, may easily be included into the thermal noise component. 
Shot noise is a noise source intrinsic to the conversion of light to electrical current in a photodiode, a quantum process where the discrete nature of photons and electrons creates an observable fluctuation [107]. Shot noise is independent of frequency to a point well beyond the signal bandwidth, and it causes a photocurrent variance given by

$$
\sigma_{\text {single }, \text { shot }}^{2}=q i_{\mathrm{A}, \mathrm{dc}} B=2 q \rho R_{r} P_{\text {source }} B
$$

where $q$ is the electronic charge, $B$ is the noise equivalent bandwidth of the photodetector and bandpass filter, and we have assumed that the sample arm power is neglible compared to the reference arm power.

Optical mixing noise, also known as "intensity" and "beat" noise, is present due to the fact that the coherent interference between randomly fluctuating optical fields followed by square-law optical detection results in a down-mixing of random frequency components to the detection band of the OCT signal [107-110]. Light sources emitting photons by thermal excitation or spontaneous emission may be considered "thermal" light sources and are modelled by the Bose-Einstein counting distribution. The superluminescent diode used in the constructed OCT system falls into this category.

To analyse the optical mixing we split the optical fields into coherent and incoherent components. The coherent light components are the optical fields from the sample, $u_{s c}$, and reference, $u_{r c}$, arms which have travelled exactly the optical path length corresponding to the position of the sample reflector and reference mirror. The incoherent fields, $u_{s i}$ and $u_{r i}$, are the sum of all other light reflections from the sample and reference arms. The optical power of each of the four field components can be written in terms of reflectivities $R_{s c}, R_{s i}, R_{r c}$ and $R_{r i}$, i.e.

$$
\begin{array}{r}
P_{s c, s i}=\left|u_{s c, s i}\right|^{2}=\alpha R_{s c, s i} P_{\text {source }} \\
P_{r c, r i}=\left|u_{r c, r i}\right|^{2}=(1-\alpha) R_{r c, r i} P_{\text {source }}
\end{array}
$$

These four sample and reference arm fields are combined by the fibre-optic coupler and they interfere (mix) to give the total optical power $P_{A}=1 / 2 \mid u_{r c}+u_{r i}+$ $u_{s c}+\left.u_{s i}\right|^{2}$. The fields are considered to be stationary in this analysis and each represents a stochastic field. This expression is evaluated by expansion and consideration of the sixteen terms produced. The spectral density of the current noise can then be determined by taking the Fourier transform of the auto-correlation function (by the Einstein-Weiner-Kintchine Theorem) [107,111]. Without writing 
out the full derivation here, we can calculate the total noise power arising from the optical mixing noise by examining separately the sixteen terms in the two categories of four self-correlations and twelve cross-correlations (see [75] for more details).

The four auto-correlation terms contribute optical mixing noise which can be called the "self-beat noise" or "source intensity noise". The total noise power of each of these terms is given by [107]

$$
\sigma_{\mathrm{x}, \text { self beat }}^{2}=\left(1+\mathbb{P}^{2}\right) i_{\mathrm{x}, \mathrm{dc}}^{2} \tau_{c} B
$$

where $i_{\mathrm{x}, \mathrm{dc}}$ is the DC photocurrent which would be created by this component, $\mathbb{P}$ is the degree of polarization of the field ( $\mathbb{P}=1$ for polarized light), and $\tau_{c}$ is the coherence time,

$$
\tau_{c}=\left(\int_{0}^{\infty} g^{2}(\nu) d \nu\right)^{-1}
$$

where $g(\nu)$ is the normalized power spectral density of the light source.

The twelve cross-correlation terms can be reduced to six pairs of terms by recognizing that each cross-correlation term has a corresponding complex conjugate. One pair of fields, $u_{r c}$ and $u_{s c}$, which give the terms $u_{r c} u^{*}{ }_{s c}$ and $u^{*}{ }_{r c} u_{s c}$, are mutually coherent, by definition, and result in the desired OCT signal (Equation 3.7). The remaining five pairs of cross-correlation terms contribute noise. We make the assumption that there is negligible filtering of the optical spectrum, dispersion or birefringence. Since they arise from the same source the characteristics of the fields $u_{r c}, u_{r i}, u_{s c}, u_{s i}$ are identical apart from scaling factors and time shifts. Goodman shows that the total current noise power contributed from the mixing of two identically-polarised fields $u_{\mathrm{x}}$ and $u_{\mathrm{y}}$ arising from the same source but which are mutually incoherent can be expressed in terms of the DC photocurrent each field component produces [107]:

$$
\sigma_{\mathrm{x}, \mathrm{y} \text { beat }}^{2}=2 i_{\mathrm{x}, \mathrm{dc}} i_{\mathrm{y}, \mathrm{dc}} \tau_{c} B
$$

Applying this to all of the cross-correlation terms, combining with the auto-correlation terms, and expressing in terms of reflectivities, the total optical mixing noise 
power is given by

$$
\begin{aligned}
\sigma_{\text {single }, \text { mixing }}^{2} & =\left\{\alpha^{2} R_{s i}^{2}+(1-\alpha)^{2} R_{r c}^{2}+(1-\alpha)^{2} R_{r i}^{2}\right. \\
& \left.+2 \alpha(1-\alpha)\left[R_{r c} R_{r i}+R_{r c} R_{s i}+R_{r i} R_{s i}\right]\right\} \frac{1}{2} \rho P_{\text {source }} \tau_{c} B .
\end{aligned}
$$

Here we have assumed that $R_{s c}$ is small compared to $R_{s i}$ so that it does not contribute significantly to the incoherent sample reflectivity component when the reference delay scan has moved beyond the sample location.

Thermal (Nyquist) noise is the fluctuation in electrical current caused by thermally-excited electrons in any conductor with non-zero resistance. The thermal noise power in the photocurrent is

$$
\sigma_{\text {single,thermal }}^{2}=\frac{4 k T}{R_{t}} B
$$

where $k$ is Boltzmann's constant, $T$ is the temperature of the conductor and $R_{t}$ is the resistance seen by the photocurrent.

$S N R$ The single-ended configuration SNR is then

$$
S N R=\frac{P_{\text {single,signal }}}{\sigma_{\text {single,shot }}^{2}+\sigma_{\text {single,thermal }}^{2}+\sigma_{\text {single,mixing }}^{2}}
$$

We solve SNR $=1$ for $R_{\mathrm{sc}}$. The solution can be written as a sum of the individual contributions to the sensitivity

$$
\begin{aligned}
R_{\mathrm{sc}, \min } & =\frac{\sigma_{\text {single,shot }}^{2}+\sigma_{\text {single,thermal }}^{2}+\sigma_{\text {single,mixing }}^{2}}{(1 / 2) \alpha(1-\alpha) \rho R_{\mathrm{rc}} P_{\text {source }}^{2}} \\
& =R_{\text {shot,single }}+R_{\text {thermal,single }}+R_{\text {mixing,single }}
\end{aligned}
$$

This total sensitivity and the contributions will be plotted in comparison with the dual balanced configuration expressions below.

3.4.2 Dual balanced configuration As shown in Figure 3.11(b) the output of the dual-balanced OCT configuration, $i_{A}-i_{B}$, is the bandpass-filtered difference between the signal from the two outputs of the fibre coupler. The main advantage of a dual balanced configuration is that most of the optical mixing noise is eliminated [111]. All of the source intensity noise (self-beat) terms are identical at both outputs, thus they are eliminated by the subtraction $i_{A}-i_{B}$. The same also applies to the optical mixing between components which arise from the same side of 
the interferometer, i.e. $u_{r c} u_{r i}^{*}$ and $u_{s c} u_{s i}^{*}$. The elimination of these common-mode noise components is not perfect due to imbalance in the fibre coupler and subtraction circuit; the actual reduction is measured by the common-mode rejection ratio (CMRR). This technique was previously used in coherent communication receivers to reject local oscillator noise [112].

Optical splitting couplers impart a $\pi / 2$ phase shift to the optical fields which couple from one guide to another relative to optical fields that remain in the original guide. This means that the components of $i_{A}$ and $i_{B}$ which result from optical mixing between fields from opposite sides of the interferometer will have opposite signs in $i_{A}$ and $i_{B}$. Therefore, when the difference $i_{A}-i_{B}$ is formed, those components double in amplitude and this results in a factor of four increase in power. The shot and thermal noise processes in $i_{A}$ and $i_{B}$ are uncorrelated, therefore we sum their noise powers to find the total shot and thermal noise powers in $i_{A}-i_{B}$. The resulting noise and signal powers are then

$$
\begin{aligned}
P_{\text {dual,signal }} & =4 P_{\text {single,signal }} \\
\sigma_{\text {dual,shot }}^{2} & =2 \sigma_{\text {single,shot }} \\
\sigma_{\text {dual,thermal }}^{2} & =2 \sigma_{\text {single,thermal }} \\
\sigma_{\text {dual,mixing }}^{2} & =\left\{\frac{1}{C M R R}\left[\alpha^{2} R_{s i}^{2}+(1-\alpha)^{2} R_{r c}^{2}+(1-\alpha)^{2} R_{s i}^{2}\right]\right. \\
& \left.+8 \alpha(1-\alpha)\left[R_{r c} R_{r i}+R_{r c} R_{s i}+R_{r i} R_{s i}\right]\right\} P_{\text {source }}^{2} \tau_{c} B
\end{aligned}
$$

The equation $S N R=1$ can then be solved to give the sensitivity in the same manner as for the single-ended configuration.

3.4.3 Maximising sensitivity of the OCT system The sensitivity expressions indicate that a greater sensitivity should be achieved with a dual-balanced interferometer configuration due to the cancelling of the common-mode intensity noise. Figure 3.12 demonstrates this with plots of the total sensitivity versus refence reflectivity $\left(R_{r c}\right)$ for both configurations, with contribution to the sensitivity of the individual noise components.

Figure 3.13(a) is a 3D surface plot of the sensitivity versus source power and reference reflectivity, and Figure 3.13(b) shows a plot of the sensitivity versus source power, where the optimum reference reflectivity has been used at every point. The plots show that above a certain level of available optical power, the reference arm needs to be attenuated to achieve optimum sensitivity.

A practical issue requiring consideration for interferometer implementation is 

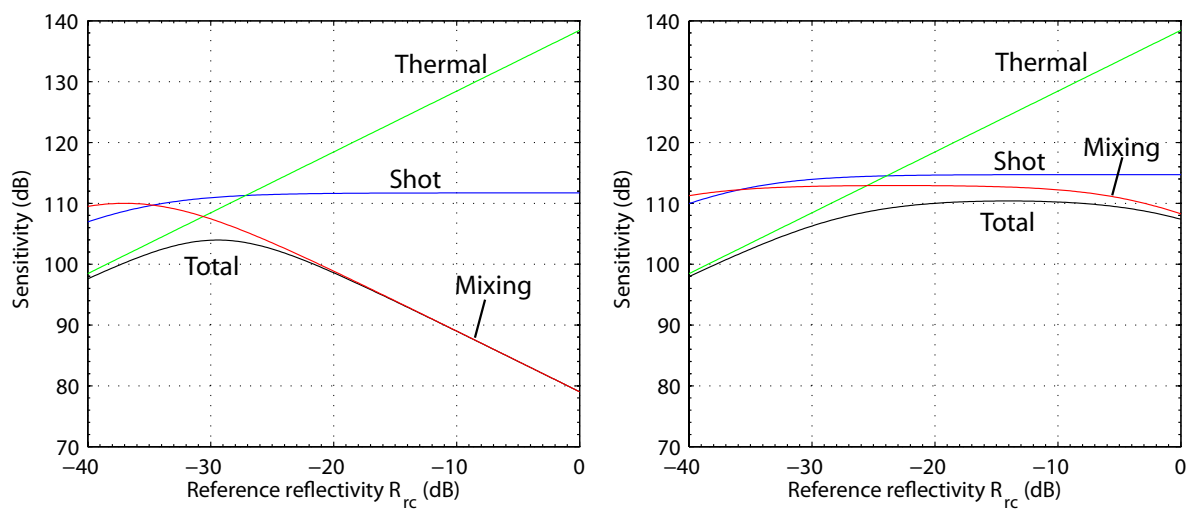

Figure 3.12: OCT sensitivity for the (a) single-ended configuration and (b) dual balanced configuration. The configuration parameters are $\lambda_{0}=$ $1300 \mathrm{~nm}, \Delta \lambda=50 \mathrm{~nm}, \alpha=0.5, R_{r i}=-40 \mathrm{~dB}, R_{s i}=-40 \mathrm{~dB}, B=$ $100 \mathrm{kHz}, C M R R=25 \mathrm{~dB}, R_{t}=10 \mathrm{k} \Omega$.
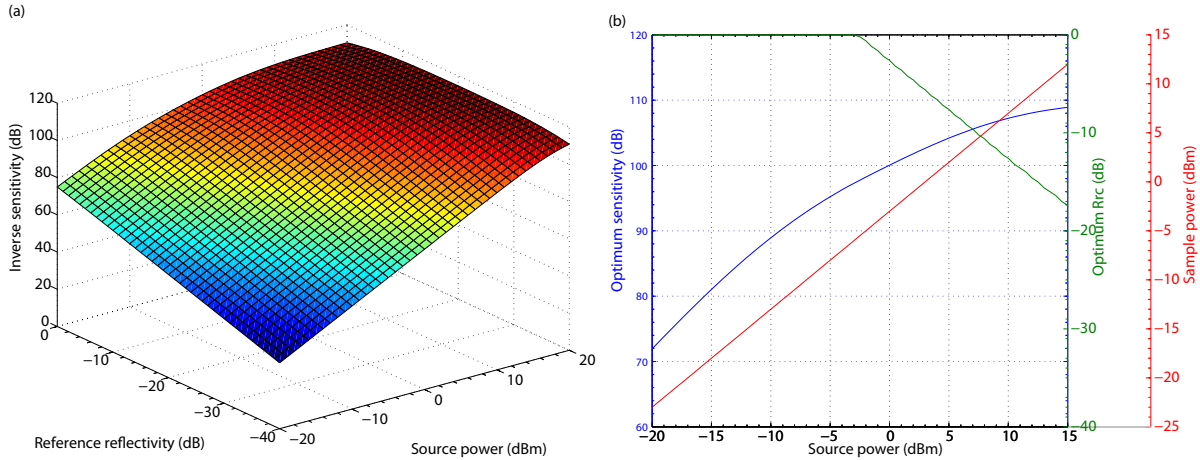

Figure 3.13: (a) OCT sensitivity versus source power $\left(P_{\text {source }}\right)$ and $R_{r c}$ (b) best sensitivity (i.e. $R_{r c}$ set to the optimum value) versus $P_{\text {source }}$. Also plotted are the optimum reference reflectivity and power at the sample. The configuration is dual balanced with the same parameters as Figure 3.12.

the achievable insertion loss. Figure 3.13(b) shows that (for that particular set of parameters) the optimum reference reflectivity is $0 \mathrm{~dB}$ when the source power is less than $-3 \mathrm{dBm}$. However, with a long-range FDODL and an electro-optic phase modulator, it is not unreasonable to suffer a total of $10 \mathrm{~dB}$ loss in the reference arm. This will reduce the achievable sensitivity, as shown in Figure 3.14(a). Figure 3.14(b) shows the gain in sensitivity from the single-ended configuration to the dual-balanced configuration, where it is assumed the optimum (within the available range) reference reflectivity is always used. This is shown for both the case of zero reference arm loss and the case of a reference arm loss which is $10 \mathrm{~dB}$ or greater. The difference between the two cases is significant for a source power of $5 \mathrm{dBm}$ or less. Nevertheless, in both cases there is a clear sensitivity advantage 
in dual-balanced detection.
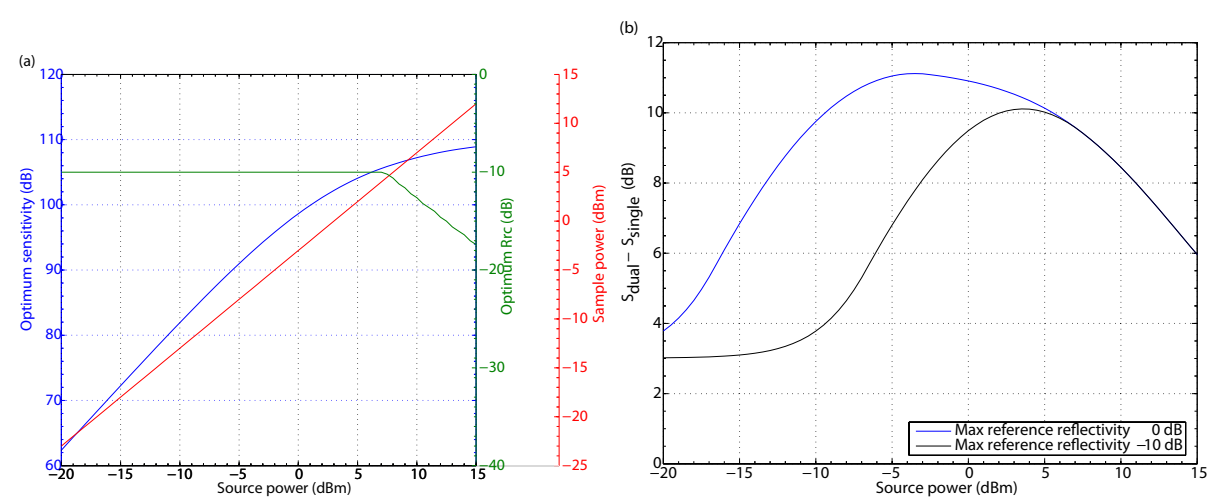

Figure 3.14: (a) OCT sensitivity versus source power $\left(P_{\text {source }}\right)$ and $R_{r c}$ (b) best sensitivity (i.e. $R_{r c}$ set to the optimum value) versus $P_{\text {source }}$.

3.4.4 Limited sample power. OCT systems are most often used on living biological tissue, and appropriate care must be taken to avoid permanent damage to the sample material by irradiation with excessive optical power. A maximum permissable exposure (MPE) limit is calculated using the relevant various laser safety standards, for example the ASNZ [113] and ANSI [114] standards. For a focussed spot with diameter $180 \mu \mathrm{m}$ the limit for continuous exposure to skin is $0.25 \mathrm{~mW}$. When scanning the sample beam across the tissue a higher sample arm power level may be used, for example at $75 \mathrm{~mm} / \mathrm{s}$ the limit is $125 \mathrm{~mW}$. However for the eye safety of the patient and operator it is preferable to restrict the power to a safer level, e.g. $5 \mathrm{~mW}$. The restriction in sample power restricts the achievable system sensitivity. If the sample power should be limited to $5 \mathrm{~mW}$ then the source power is limited to $10 \mathrm{~mW}$ (for a balanced coupler). As shown by Figures 3.13 and 3.14, limiting the optical power limits the achievable sensitivity. If the sensitivity is limited by the optical mixing noise, however, additional power does not increase sensitivity. This is due to the fact that both the signal power and optical mixing noise are proportional to the square of the source power.

3.4.5 Variable splitting ratio The results shown above are based on interferometers with balanced (50:50) couplers, ie, $\alpha=0.5$. The use of other splitting ratios can result in different sensitivities. It has been shown that a sensitivity advantage can be achieved by optimizing the splitting ratio as well as the reference arm attenuation [115]. Figure 3.15 shows the variation in sensitivity versus the splitting ratio for the single-ended and dual-balanced configuration. 

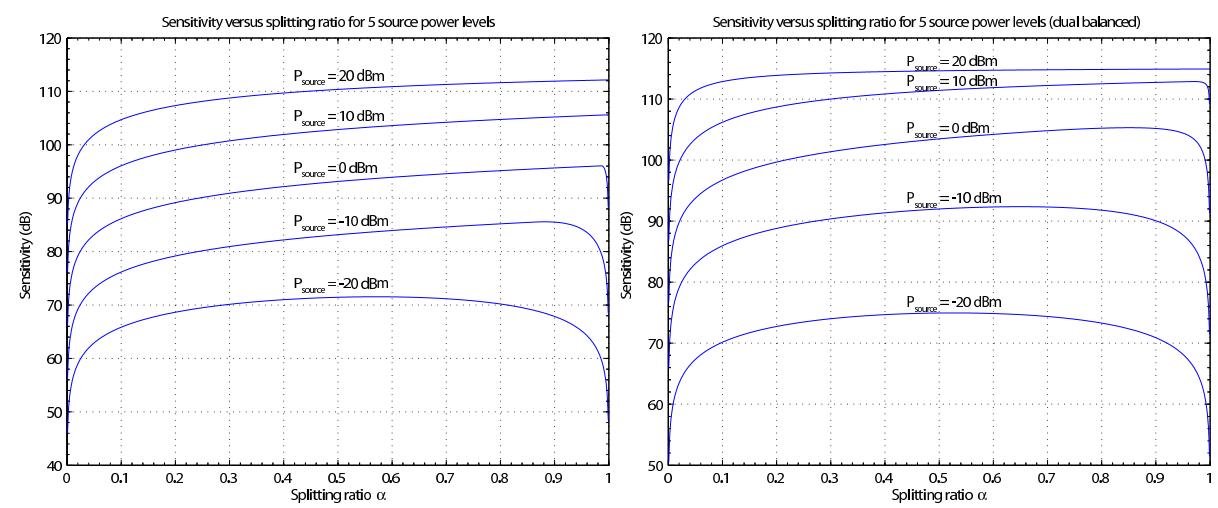

Figure 3.15: Sensitivity versus splitting ratio for different source power levels, for single-ended and dual-balanced configurations. The other parameters are the same as in the previous plots.

Figure 3.16 shows the level of improvement using an unbalanced coupler versus the available source power. At every point the optimum splitting ratio and reference reflectivity are used. When a low loss reference arm is available in a single-ended detection OCT system, the use of an optimised coupling ratio gives a sensitivity advantage greater than $1 \mathrm{~dB}$ for source power greater than $-16 \mathrm{dBm}$ (up to some value beyond $20 \mathrm{dBm}$ ). For the case of a limited maximum reference reflection the source power must greater than $-6 \mathrm{dBm}$ for the same level of improvement. The plot shows that the level of improvement is at most $3 \mathrm{~dB}$. For dual-balanced detection the value of going to an unbalanced coupler is even less. For the $a \mathrm{OCT}$ application this does not justify the extra cost and complexity of the coupler with a specific coupling ratio.

3.4.6 Sensitivity-resolution tradeoff The bandpass filter at the interferometer output (Figure 3.11) is an integral part of the system. It is required to reject noise which is outside the bandwidth of the OCT signal. A narrower filter rejects more noise, though the bandwidth should not be so small that it significantly filters the signal. Hee discussed the tradeoff in OCT system design [116] and noted that the sensitivity and depth resolution $(\Delta l)$ are inversely related,

$$
\frac{S N R_{\text {shot-noise-limited }}}{\Delta l}=\frac{\eta}{h \nu} \frac{\pi}{8 \ln 2} \frac{P_{\mathrm{sc}}}{v}
$$

In practice it is not possible to simultaneously achieve the resolution and sensitivity predicted by this equation. This is the case as a Gaussian signal profile is not band-limited, therefore any bandpass filtering will cause an increase in pulse width. To achieve maximum sensitivity the optimum method is the use 


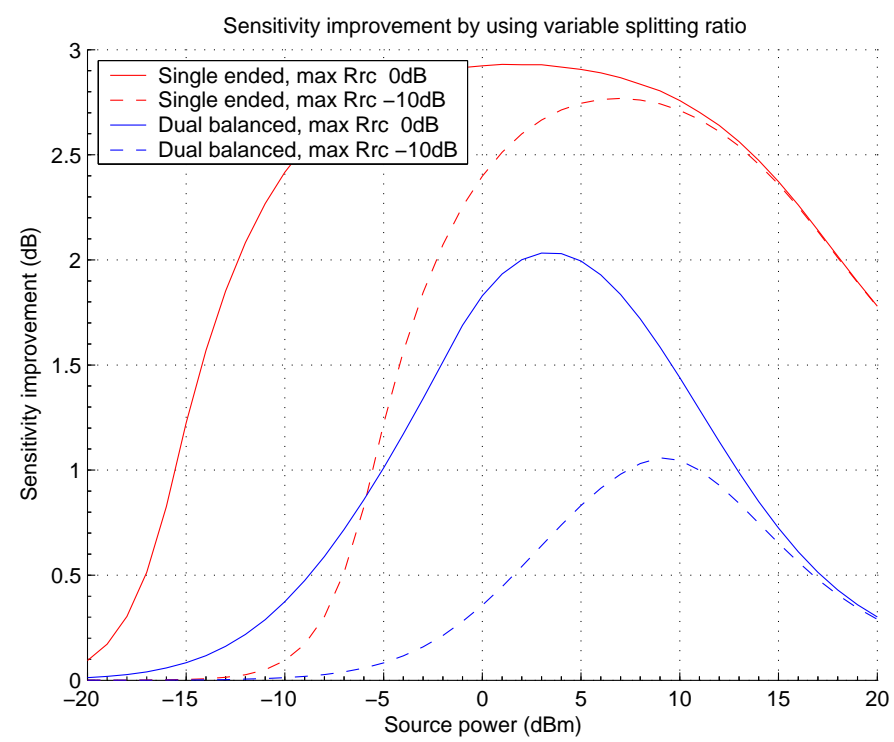

Figure 3.16: Optimum sensitivity versus source power for single-ended and dual-balanced configurations, with and without a $10 \mathrm{~dB}$ loss in the reference arm.

of a matched filter to attain maximum SNR; in the case of a Gaussian coherence function this is a filter with a Gaussian response. The relationship between the resolution, sensitivity and filter width can be calculated from the response of the Gaussian filter to a Gaussian pulse, where the two have the same centre wavlength but different widths:

$$
\begin{aligned}
\frac{\Delta l_{\text {out }}}{\Delta l_{\text {in }}} & =\sqrt{1+1 / \beta^{2}} \\
\frac{S N R_{\text {out }}}{S N R_{\text {in }}} & =\frac{2}{\beta+1 / \beta}
\end{aligned}
$$

and $\beta$ is the ratio between the filter and signal bandwidths, $\Delta f_{\text {filter }} / \Delta f_{\text {signal }}$.

Figure 3.17 shows graphically the tradeoff between pulse broadening and sensitivity. The use of a filter with width $2 \Delta f$ to maintain axial resolution for a small SNR penalty has been suggested [91]. In the present case of the $a \mathrm{OCT}$ system the axial resolution requirement is relatively modest, therefore it would be reasonable to compromise axial resolution to achieve the maximum sensitivity, therefore the filter width should be set to $\Delta f$ instead $(\beta=1)$.

3.4.7 Fourier-domain OCT Fourier-domain OCT (FD-OCT) is a form of optical coherence tomography where the sample reflectivity profile is determined by analysing the spectral response of the interferometer rather than scanning the 


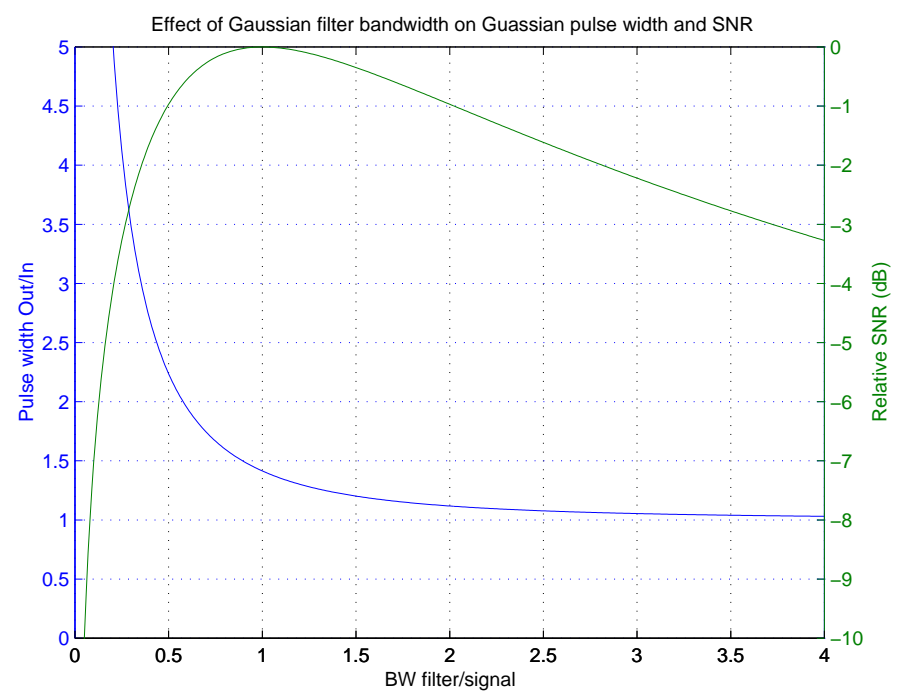

Figure 3.17: Simulated OCT depth resolution and sensitivity penalty versus the ratio of filter to signal bandwidth. The signal and filter shape are Gaussian.

reference arm distance. It has been shown theoretically and experimentally that FD-OCT methods allow a greater sensitivity to be achieved than time-domain OCT $[117,118]$. There are two types of FD-OCT which are essentially equivalent: (a) "spectral-domain OCT" (SD-OCT), where the optical spectrum of the interferometer output is measured at one point in time by a spectrometer, and (b) "sweptsouce OCT" (SS-OCT), where a narrow linewidth source is swept through a range of frequencies and the interferometer output intensity measured during the entire sweep. With the same optical bandwidth parameters, the distance ranging resolution is the same for these techniques as for TD-OCT.

When shot-noise limited, SD-OCT has a sensitivity advantage over TD-OCT of a factor of $M$, the number of spectrometer pixels [117,118]. SD-OCT requires no moving components to capture an A-scan, only a single spectrum captured with the spectrometer sensor (eg. a single-line CCD). Also, the system can be kept quite compact as a scanning optical delay line is unneccesary. These are both very attractive features for clinical instruments, however, for high resolution over a long range, SD-OCT has a limitation compared to SS-OCT in that the total distance range is limited by the number of spectrometer pixels. The maximum distance range (in air) of each A-scan is given by [118]

$$
L_{\max }=\frac{\lambda_{0}^{2}}{4 \delta \lambda}=\frac{\pi N l_{c}}{16 \log (4)}
$$


where $\lambda_{0}$ is the centre wavelength, $\delta \lambda$ is the spectrometer resolution, $N$ is the number of pixels, $l_{c}$ is the coherence length and the FWHM of the optical spectrum occupies half of the spectrometer range. As an example, to maintain an axial resolution of $22 \mu \mathrm{m}$, for a range of $25 \mathrm{~mm}$ the spectrometer sensor would need $N=8023$ pixels; sensors of this size are currently relatively expensive. Alternatively, a degradation of axial resolution could be traded for a reduction in the number of pixels in the spectrometer sensor. This is done by reducing the total optical bandwidth captured by the spectrometer and thus fewer pixels are required. For example, for a resolution of $100 \mu \mathrm{m}$ only 1766 pixels would be required for a $25 \mathrm{~mm}$ range. Note that without an extra phase modulation element in the interferometer, the phase conjugate ambiguity means that the number of pixels required is doubled [119]. Other issues with SD-OCT is the problem of sensitivity decay along the axial measurement range, approximately $14 \mathrm{~dB}$ is a typical reported value $[119,120]$, and that SD-OCT is susceptible to "phase washout" when the sample moves during a measurement (this would be a fairly common occurance in the upper airway) [121].

SS-OCT also has a similar sensitivity advantage over TD-OCT. For a source swept over the same optical bandwidth (and constant optical intensity over that range), $S_{\mathrm{SS}-\mathrm{OCT}} / S_{\mathrm{TD}-\mathrm{OCT}}=M / 2$ where $\mathrm{M}$ is the number of samples per sweep [117]. If the laser source is frequency-tuned by non-mechanical means, the technique also does not require moving parts to perform an A-scan. The limit on distance range is determined by the number of intensity samples per sweep of the source wavelength, as with SD-OCT $L_{\max }=\lambda_{0}^{2} /(4 \delta \lambda)$, where $\delta \lambda$ is now the spacing of the samples as the wavelength is swept. Therefore, the maximum sampling rate of the data acquisition hardware should be the practical limit on the distance range. For example, for a SS-OCT system with a centre wavelength of $1300 \mathrm{~nm}$, wavelength scanning range of $34 \mathrm{~nm}$ and an A-scan rate of $1 \mathrm{kHz}$, to achieve $25 \mathrm{~mm}$ the required sampling rate (at the Nyquist limit) is $2 \mathrm{MHz}$, which is easily achievable with standard high-speed data acquisition cards. When the $a \mathrm{OCT}$ system was initially being designed, SS-OCT was first chosen as the technique for distance ranging, though at the time the technique was called frequencymodulated continuous-wave interferometry (FMCW). Appendix A contains a paper containing some results of the initial investigation of the use of FMCW ranging [122]. The main disadvantage of SS-OCT at that time (circa 1999) was the limited availability of suitable tunable sources, and this was the reason that SSOCT was not finally used for the $a \mathrm{OCT}$ system. However, recently the situation 
has improved as the realization of the benefits of SS-OCT has prompted the development of tunable laser sources marketed specifically for OCT (for example, the SL1325-P16 laser from Thorlabs, NJ, USA).

\subsection{Summary}

This chapter began with the explanation of the necessity for a rapid long range delay line for the $a \mathrm{OCT}$ system. The operation of the frequency domain optical delay line was briefly explained and several variations on the delay line configuration described. The results of optical modelling analysis presented showed that for the aOCT system the out-of-plane, on-pivot configuration was the best in terms of achievable delay range. This choice necessitates the use of a phase modulator in the interferometer as the delay line would give zero phase modulation.

OCT sensitivity modelling was necessary to achieve optimum image contrast as the $a \mathrm{OCT}$ probe has a very low numerical aperture and thus low light collection efficiency, and the available source power was limited due to cost and size restrictions.

It was shown that, as achieving the best possible axial OCT resolution is not important for aOCT scanning, the best bandpass and lowpass filter bandwidth to use was that matching the signal bandwidth. The signal bandwidth is calculated from the system scaning speed and optical bandwidth. Finally, the spectraldomain and swept-source OCT techniques were compared to the currently used technology, time-domain OCT, in terms of sensitivity and scanning range. Sweptsource OCT is identified as a very good candidate for practical improvements in the instrument if a suitable tunable laser source is available. 


\section{System design}

The purpose of this chapter is to present the engineering design of the portable aOCT system for hospital-based measurements. The design of the OCT system hardware is described, particularly the probe design. This is followed by the signal processing electronics, software, integration with other sleep equipment dataloggers, and finally data processing. The techniques include the procedures investigated to enhance the $a \mathrm{OCT}$ images, extract lumen contours of the hollow organs, and generate three-dimensional reconstructions. Part of the work reported in this chapter was carried out in collaboration with Matthew Leigh (particularly probe fabrication, software, microprocessor control, data processing).

\subsection{System layout and interferometer}

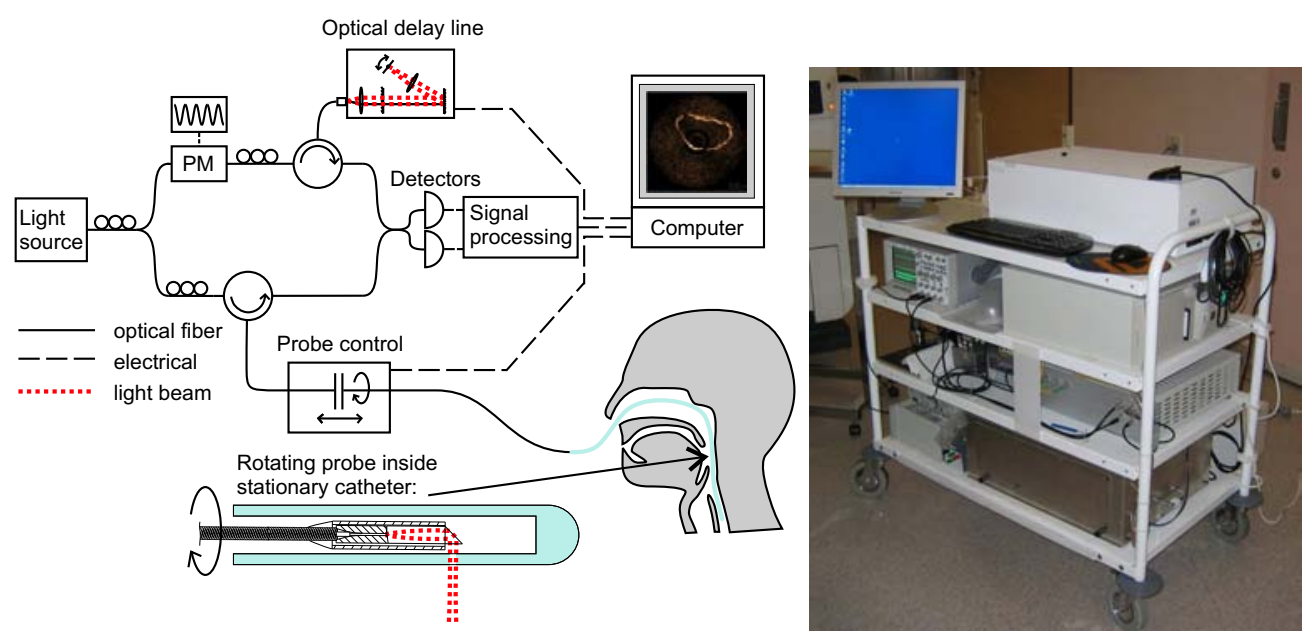

Figure 4.1: Schematic diagram and photograph of the $a \mathrm{OCT}$ system. PM - phase modulator.

A schematic diagram showing the overall design of the $a$ OCT system is shown in Figure 4.1. The core of the OCT system is the fibre-optic inteferometer which is in a Mach-Zehnder configuration. This configuration is a power-efficient design allowing a high SNR to be reached [81].

The reference arm is terminated in a frequency-domain optical delay line using the out-of-plane, on-pivot design, as described in Chapter 3. The range of the the delay line is $27 \mathrm{~mm}$, and a scan repetition rate of $250 \mathrm{~Hz}$ is used (limited by the FDODL galvanometer). The reference arm contains an electro-optic (LithiumNiobate) phase modulator which serrodyne modulates the phase to create an optical frequency shift of $1.2 \mathrm{MHz}$. The use of the Mach-Zehnder configuration 
means that light passes only once through the phase modulator, a relatively high loss component $(7 \mathrm{~dB})$, rather than two times which would occur if a Michelson configuration were to be used.

The light source is a superluminescent diode (Denselight, Singapore). The SLD centre wavelength is $1301 \mathrm{~nm}$, and the Gaussian-shaped spectrum has a FWHM bandwidth of $34.1 \mathrm{~nm}$, giving an axial OCT resolution of $22 \mu \mathrm{m}$. The power of the SLD is $21 \mathrm{~mW}$ with a high level of polarization. The light is split equally between the sample and reference arms, although the losses in each arm are different. The power incident on the sample is $4 \mathrm{~mW}$, which is below the recommended limit on skin tissue [113] for the given wavelength and speed that the beam is scanned across skin tissue (for a probe-tissue radius of $10 \mathrm{~mm}$ the maximum power is $125 \mathrm{~mW}$ as long as the beam is scanned continously). Although the incident power is similar in power to a typical laser pointer, with a long exposure the light could be potentially hazardous to eyesight - for safety, the SLD is only activated once the probe is in the airway and rotating.

The complete system is contained on a custom-built trolley for portability (see Figure 4.1). It could be controlled remotely using a wireless link (from a distance of 15 metres), which was necessary for experiments carried out in the hospital computed tomography imaging suite to prevent unnecessary exposure to $\mathrm{x}$-ray radiation.

\subsection{Sample arm}

4.2.1 Probe design Figure 4.2 shows a diagram and photograph of the $a$ OCT probe in the interferometer sample arm. The probe consists of optical fibre terminated by a micro-optics assembly which collimates and redirects light in a beam perpendicular to the probe axis. A torque coil supports the fibre and allows the probe to be rotated and translated.

The glass ferrule has two functions - to support the optical fibre during the polishing process, and to allow the fibre to be fixed to and centred on the GRIN lens (NSG America, NJ, USA). The fibre is fixed in the ferrule by UV-cured adhesive (Norland Products, NJ, USA). A ferrule-lens alignment station was built to ensure that the two faces were parallel when fixed together, as any lateral offset in position on the GRIN lens would cause the output beam to not be parallel to the lens axis. The fibre and ferrule are both angle polished at 8 degrees to ensure a high return loss for the fibre-lens back-reflection, as in FC/APC optical fibre connectors which usually offer better than $-60 \mathrm{~dB}$ return loss. 


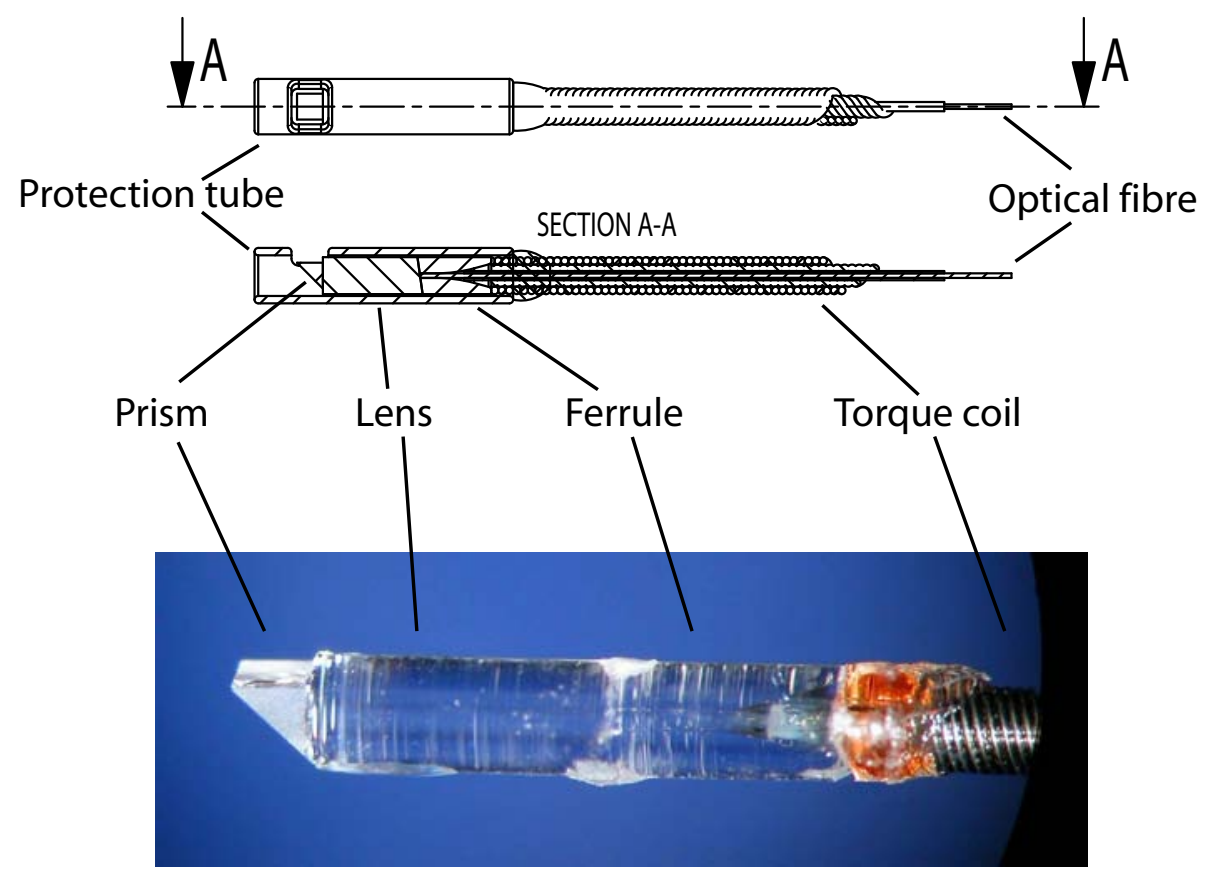

Figure 4.2: A diagram and photograph of the probe optics. The photograph shows the probe before the addition of the stainless steel protection tube.

The GRIN lens is polished to a precise length which effectively determines the focal length of the lens. The glass right-angle prism placed at the end of the GRIN lens reflects the beam (total internal reflection at the hypotenuse) perpendicularly to the long axis of the probe. Contamination at the prism hypotenuse is avoided as this will cause light to be partially transmitted at the hypotenuse. The microoptical assembly of the probe is contained inside a stainless steel sleeve (ID 1.0 $\mathrm{mm}$, OD $1.3 \mathrm{~mm}$ ) for protection, with an opening cut for the beam. Epoxy is used to fix the sleeve to the ferrule and torque coil.

4.2.2 Probe optical characteristics The function of the GRIN lens in the probe is two-fold - to collimate the light exiting the single-mode fibre, and to focus light scattering from the sample back onto the fibre core. Several key parameters of the system, (a) the transverse resolution, (b) the range of distances over which the system can detect surfaces, and (c) the sensitivity of the system, are all affected by the probe optical design. For all calculations it was assumed that the beam emitted from the fibre is Gaussian, which is a good approximation for single-mode optical fibre [123]. Also, the lens and prism are sufficiently large that the beam is not significantly truncated, therefore, Gaussian beam propagation equations are accurate. 
The optical fibre core acts like a pinhole in a confocal microscope. This means that light scattered from locations far from the beam waist will not be collected as efficiently as light scattered from near the waist. Theory predicts that maximum collection efficiency is from scattering occuring at the beam waist [124]. The axial intensity profile of the Gaussian beam is Lorentzian and characterized by the "depth of field" (DOF), the distance range around the beam waist where the beam radius is less than $\sqrt{2}$ times its value at the waist.

A large DOF is required so that scattered light is efficiently collected over a large axial range. However, increasing DOF comes at the expense of a larger beam size and therefore worse transverse resolution. This tradeoff between depth of field and transverse resolution is a common problem in OCT systems, particularly when high resolution is required over a large range of distances [75]. In this particular $a \mathrm{OCT}$ application there is not a requirement for high transverse resolution; $0.5 \mathrm{~mm}$ or less would be acceptable to be comparable to tomographic technologies such as CT or MRI. High sensitivity to enable the reliable detection of the airway surface over a long range of distances is a higher priority.
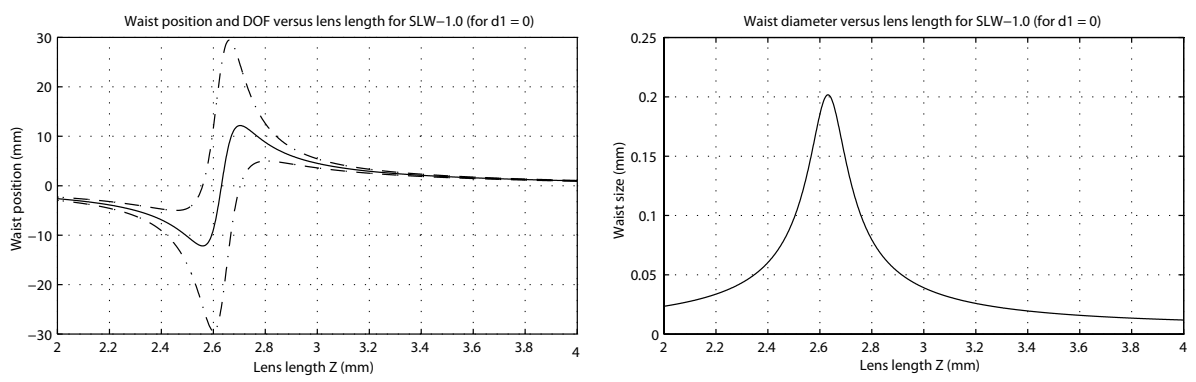

Figure 4.3: Plots for determining the GRIN lens length to achieve a maximum distance scanning response. (a) Gaussian beam waist location (solid line) and the extent of the depth of field (dashed lines). (b) Beam waist versus lens length (indicates lateral resolution).

The focussing strength of the GRIN lens is determined by its pitch (normalized lens length). It is altered by shortening the lens to the length required. The probe can be described by a simple paraxial optical model, and the ray-transfer matrix approach can be used to calculate the transmission of a Gaussian beam through the system. The lens is described by an ABCD matrix [125]. The ABCD law for Gaussian beams is then applied to calculate the location of the beam waist, and the DOF, versus the lens length [126]. Figure 4.3 shows the result of calculations for the GRIN lens (NSG SLW-1.0) and $1300 \mathrm{~nm}$ light emitted by SMF-28 fibre. The plot shows that the lens length of $2.66 \mathrm{~mm}$ will achieve the maximal distal extent of the DOF from the lens, $29 \mathrm{~mm}$. 
However, a large Gaussian beam DOF does not guarantee a higher power collection efficiency. In order to compare the power efficiencies for different lens parameters a hybrid wave/ray tracing model, implemented in Matlab, was used to calculate the power collected by the probe when a Lambertian plane reflector is placed at a series of distances away from the probe. The model operated by propagating a Gaussian beam from the fibre, through the lens, to the sample surface. To model an incoherently scattering surface, each point on the sample surface was assumed to be a point source radiating into $2 \pi$ steradians, with a total emission scaled by the Gaussian beam intensity at that point. Then ray tracing was used to calculate the solid angle of each point source emission which would be collected by the probe fibre. The condition on whether light would be coupled into the fibre was that the light should be incident on the fibre within the mode field diameter, and with an angle of incidence within the fibre angular acceptance cone. In this way an absolute power collection efficiency versus sample position could be estimated. This was repeated for each set of lens parameters.

The modelling results were used to select a NSG SLW-1.0 lens with 0.2527 pitch as the optimum value, having the best coupling efficiency response and satisfactory resolution over the desired range of $25 \mathrm{~mm}$. Figure 4.4 shows an example of modelling output for two lenses, one with pitch 0.27 and one with pitch 0.2527 . The difference in lens length between the two pitches is $0.18 \mathrm{~mm}$. The plots show that 0.27 pitch would be a better choice if the desired distance range was $15 \mathrm{~mm}$ as both the resolution and collection efficiency would be greater over that range. However, the probe transverse resolution over the axial distances $15 \mathrm{~mm}$ to $25 \mathrm{~mm}$ would be unsatisfactory and therefore to achieve a $25 \mathrm{~mm}$ range the flatter response of the 0.2527 pitch lens is preferred. Note that in this application the absolute light collection efficency will always be low because of the low numerical aperture (NA) of the probe. In the case of the above probe with flat response, NA $<0.005$. For comparison, an endoscopic OCT probe used for intra-arterial imaging [58] over a range of several millimetres had an NA of 0.039 (calculated using the reported wavelength and transverse resolution). The light collection power is proportional to the square of numerical aperture so the intraarterial probe has sixty times $(18 \mathrm{~dB})$ more signal from scattering near the focus.

4.2.3 Probe fabrication The fibre optic probes were fabricated in-house with a procedure developed to achieve low insertion loss, low parasitic back-reflections, and low beam distortion. The optical fibre (mounted in the ferrule) and GRIN lens surfaces were polished to a fine finish using polishing films with gradings 

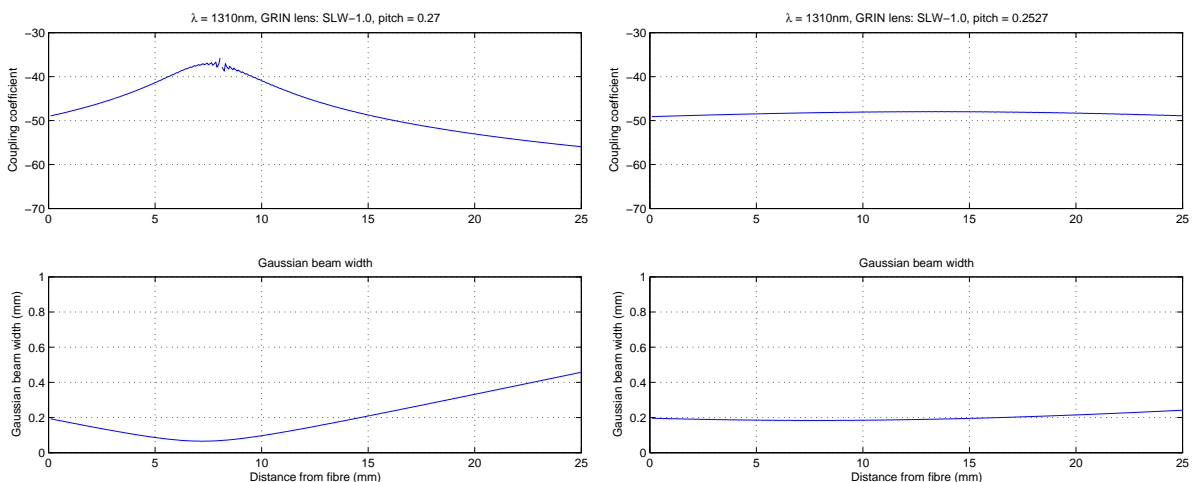

Figure 4.4: Wave/ray tracing simulation of probe coupling efficiency.

from 30 to $0.3 \mu \mathrm{m}$. A system consisting of a custom-designed tiltable polishing jig with a set of interchangeable sleds was developed to facilitate the polishing and joining stages [127]. A laser-based angle measurement station using the polishing jig was also created to ensure a consistent angle on the both the perpendicular and angled surfaces [127].

An important parameter of the fabrication process was the final length of the GRIN lens. This was monitored first by coarse length measurement during polishing using a graduated scale in the microscope eyepiece, and vernier calipers, then by an iterative process of (a) checking the probe distance-ranging intensity profile using the $a \mathrm{OCT}$ system, and (b) fine polishing to reduce the length, until a previously decided performance level was achieved. Figure 4.5 shows the probe axial response profile at three stages during the polishing of the lens. The prism was then attached and protection sleeve glued into place. An example of the typical final intensity-vs-distance profile is shown in Figure 4.6. This was created using a diffusely scattering sample in order to simulate the response of biological tissue. A Matlab script was used to determine the peak value in each A-scan and build up a profile from multiple A-scans as the object was moved through the range.

4.2.4 Probe coil design considerations The biplex torque-transmitting coil of the probes consists of two co-axial coils, each consisting of five co-wound individual wires ("filars"). The inner coil is right-handed and the outer left-handed. When the biplex torque coil is turned in an anti-clockwise direction (looking along the coil) the outer coil will contract while the inner coil will expand. The two forces oppose each other to prevent significant compression or expansion, so the torque coil does not store significant energy when torque is applied. Thus 

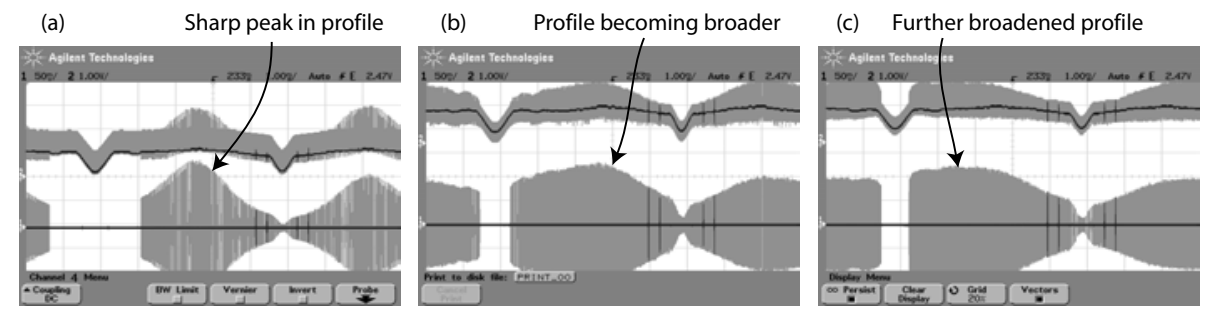

Figure 4.5: Measured probe performance at three steps during the final lens length adjustment shown by three oscilloscope screenshots. The lower trace shows the bandpass filtered ouput of the balanced photoreceiver when the sample reflector is a microscope slide (a neutral density filter is used to reduce the intensity). The images are created using the "infinite persistance" display option on a digital oscilloscope while the reflector is moved along the axial range of the probe. As the lens length is shortened towards the optimum value the profile of the maximum coupling efficiency versus distance is broadened.

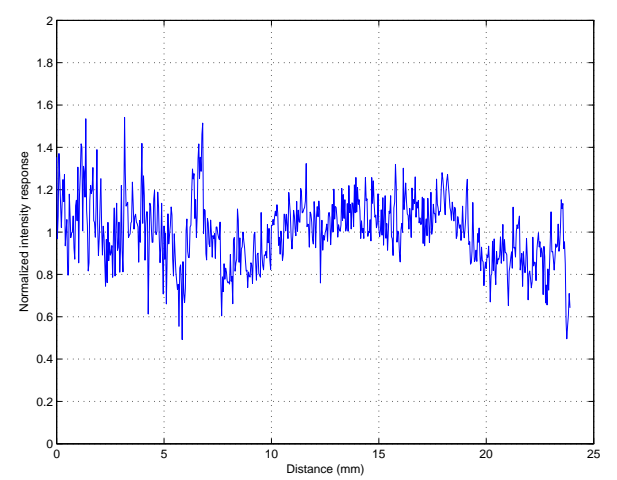

Figure 4.6: Measured probe coupling efficiency versus distance. The profile is created by moving a diffusely scattering reflector several times through the $a \mathrm{OCT}$ distance range. The fine structure in the curve is caused by speckle arising from the use of the diffuse scatterer.

the applied torque at the proximal end is transmitted along the coil rather than loading it with compressive or expansive potential energy. The torque coil retains the useful lateral flexibility of spring coils. It is possible to make a torque coil which has bi-directional torque-transmitting ability by using three co-axial coils. However, the total coil diameter would be increased, which is undesirable for the $a \mathrm{OCT}$ system. All coils must be designed so that the inner diameter is large enough to fit coated optical fibre with a diameter of $250 \mu \mathrm{m}$.

For initial experiments the total length of the probe was $1.2 \mathrm{~m}$. However, for the validation experiments simultaneously comparing $a \mathrm{OCT}$ and CT-derived measurements (see Chapter 5), new probes of length $2.35 \mathrm{~m}$ had to be fabricated so the probes could reach the patient inside the CT machine. The torque coil 
length is that of the probe minus the lengths of the FC optical connector and the optical head.

The ideal torque coil for the $a \mathrm{OCT}$ probe would be one which perfectly transmits torque to the distal end independently of the bending of the probe, and resists kinking and bending when force is applied to push the probe further into the catheter. See Figure 4.7 for a graphical illustration. The variable parameters of the torque coil design are the metal composition of the filars, the diameter of the filar wire, and the number of filars per coil. The key characteristics of a torque coil are the stiffness of the coil and the efficiency of torque transfer along it when it is constrained inside a catheter. The efficiency of torque transfer through the coil is a critical parameter, as it affects the constancy of the probe head rotation. Rotation speed fluctuation causes image distortion. This problem has been noted in intravascular ultrasound systems, which also use torque cables [128]. Coil stiffness affects how smoothly the coil can be pushed forward through catheter. The maximum speed the coil can be translated is limited by the coil stiffness and rotation speed.
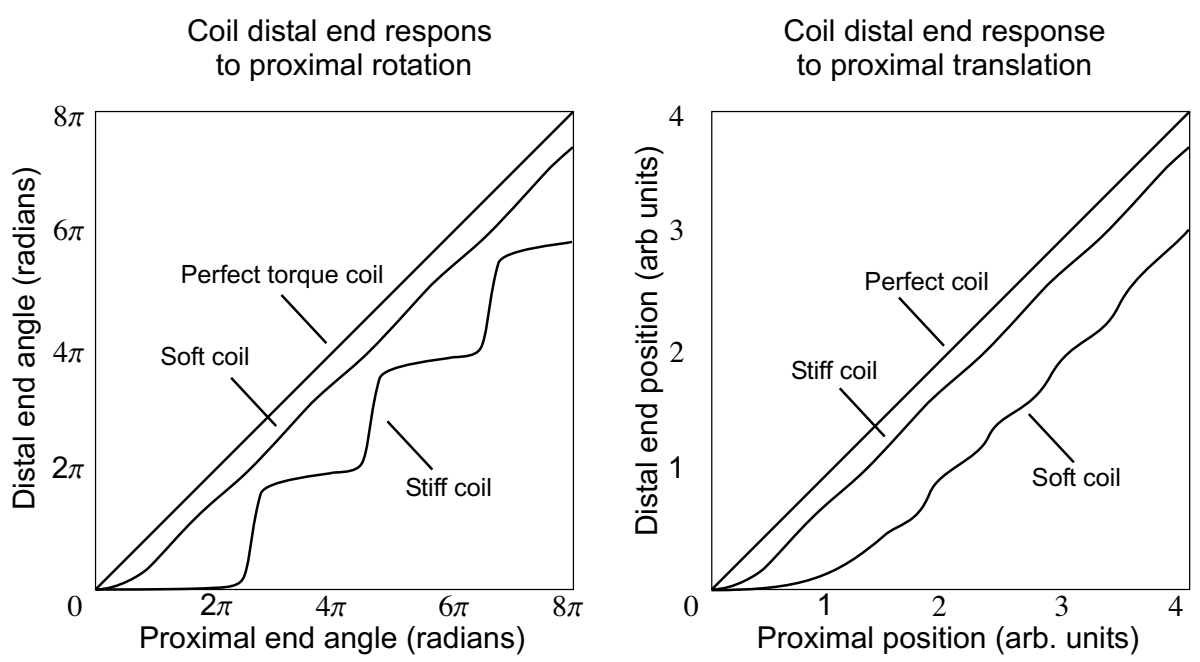

Figure 4.7: Two key torque coil properties for the aOCT probe - response to rotation and translation.

The design which was finally found to be the best performer of a series of prototypes was a hybrid coil consisting of two $1.2 \mathrm{~m}$ sections of different torque coils. The proximal end coil section was stiffer to facilitate transfer of the pulling and pushing force, and the distal end part was a softer coil which was far superior at transmitting rotational torque around a curved path (as is encountered in the upper airway). The stiff torque coil was constructed of 0.008 inch diameter stainless steel wire wound in five-filar coils for both the inner and outer coils. The soft coil 
had individual wires 0.0035 inch in diameter in the same arrangement. During tests it was found to be essential to keep the stiff section of the probe straight to avoid non-uniform rotation and image distortion. Figure 4.8 shows examples of image distortion arising from paths of the catheter which have curvature exceeding the design limits of the coil.

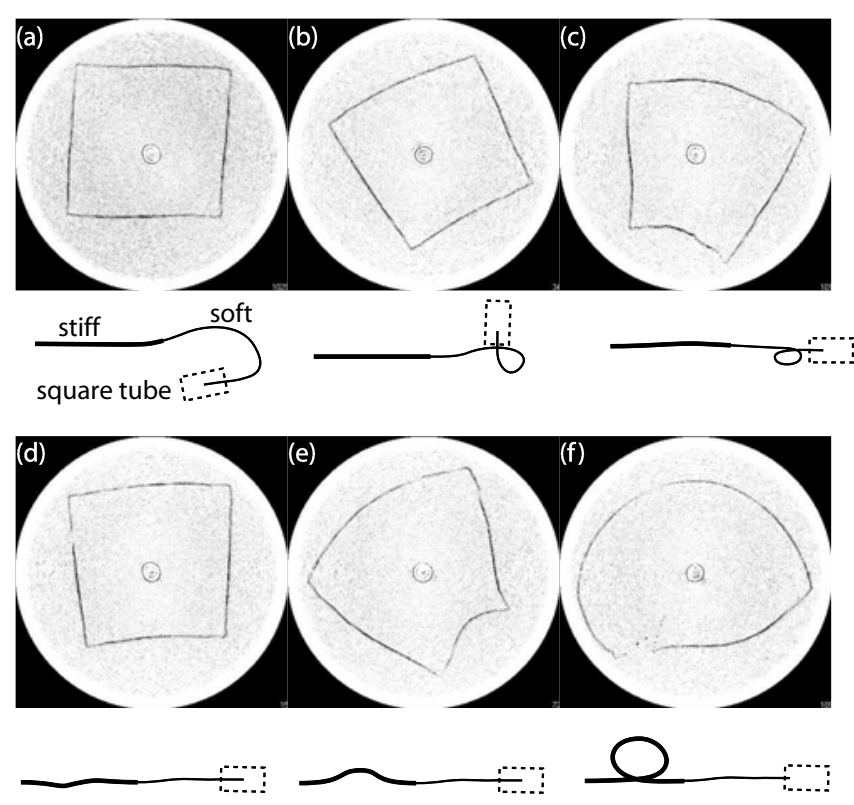

(g)

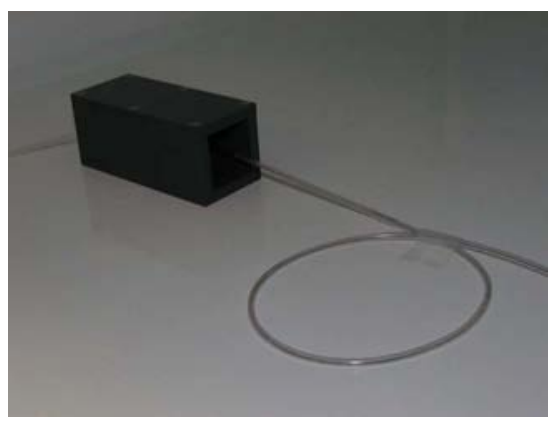

Figure 4.8: aOCT images of a square tube for a set of curved catheter paths. Distortion is caused by non-uniform distal end rotation. All images are $52 \times 52 \mathrm{~mm}$. (a) Shallow bends of cathter in distal part of the catheter (soft coil type), similar to that encountered in airway. (b) Catheter curved around $270^{\circ}$ of a $30 \mathrm{~mm}$ radius circle in the distal part. (c) Catheter curved around $360^{\circ}$ of a $15 \mathrm{~mm}$ radius circle in distal part. (d) Catheter with shallow bends in proximal part (stiff coil type). (e) Catheter with $60^{\circ}$ of $400 \mathrm{~mm}$ radius bend in proximal part. (f) $360^{\circ}$ of $50 \mathrm{~mm}$ radius bend in proximal part. (g) Photo of probe in the test object for angular distortion measurement.

A set of coils encased in teflon (PTFE) were tested to determine if frictionreduction between the coil and catheter would reduce angular-velocity distor- 
tion. This was not successful and the results suggested that friction between the metal coil and the catheter wall was significantly less without the coating, possibly because the surface area of contact was much greater with the coating present.

4.2.5 Catheter The catheter of the aOCT system is the component which physically contacts the subject. It consists of transparent medical-grade PVC tubing (AlphaGary, Massachusetts, USA) with a proximal end Luer lock connecter and terminated at the distal end with a rounded plug made from silicone adhesive (Dow Corning). The tubing has an outer diameter of $3 \mathrm{~mm}$ and inner diameter of $2 \mathrm{~mm}$, allowing sufficient clearance for the $1.3 \mathrm{~mm}$ probe. Curvature of the PVC tubing (due to storage on a reel after manufacture) was corrected by hanging the completed catheter with a weight on the end for 24 hours.

The inner diameter of the catheter tubing limits how tightly the catheter can be bent before the inner tubing wall applies significant pressure to the probe head. This would cause fluctuations in the rotation rate, and possibly damage to the probe. A simple geometric calculation is used to calculate the minimum bend radius of the catheter. Given a tubing inner diameter of $d_{t}$ and probe dimensions $l_{p}$ and $d_{p}$ (length and diameter), the radius from the centre of the catheter bend to the innermost (with respect to the bend) internal surface of the tubing is given by

$$
r_{t}=\frac{l_{p}^{2} / 4+d_{p}^{2}-d_{t}^{2}}{2\left(d_{t}-d_{p}\right)} .
$$

For $d_{p}=1.3 \mathrm{~mm}, l_{p}=10 \mathrm{~mm}$, and $d_{p}=2 \mathrm{~mm}$, then the minimum bend radius is $16 \mathrm{~mm}$. The curvature of the catheter path around the upper airway is typically the greatest at the upper nasopharynx where the airway path turns through 90 degrees. Figure 4.9 shows an x-ray CT section of a volunteers head and neck with an $a \mathrm{OCT}$ catheter and probe in place. Radii of curvature are marked along the curved parts of the catheter path. The diagram shows that the above probe and catheter parameters are sufficient to prevent the probe being trapped by the catheter bends. Also, in some clinical experiments involving the use of a CPAP mask an airtight seal between the mask and skin is required. Then the catheter is taped down to the cheek and consequently the catheter path has a tight bend at the external nares, approximately $25 \mathrm{~mm}$ bend radius.

\subsection{Electronic signal processing}

The OCT signal from the balanced photoreceiver is amplified (Minicircuits low-noise amplifier), bandpass filtered and input to a logarithmic envelope de- 


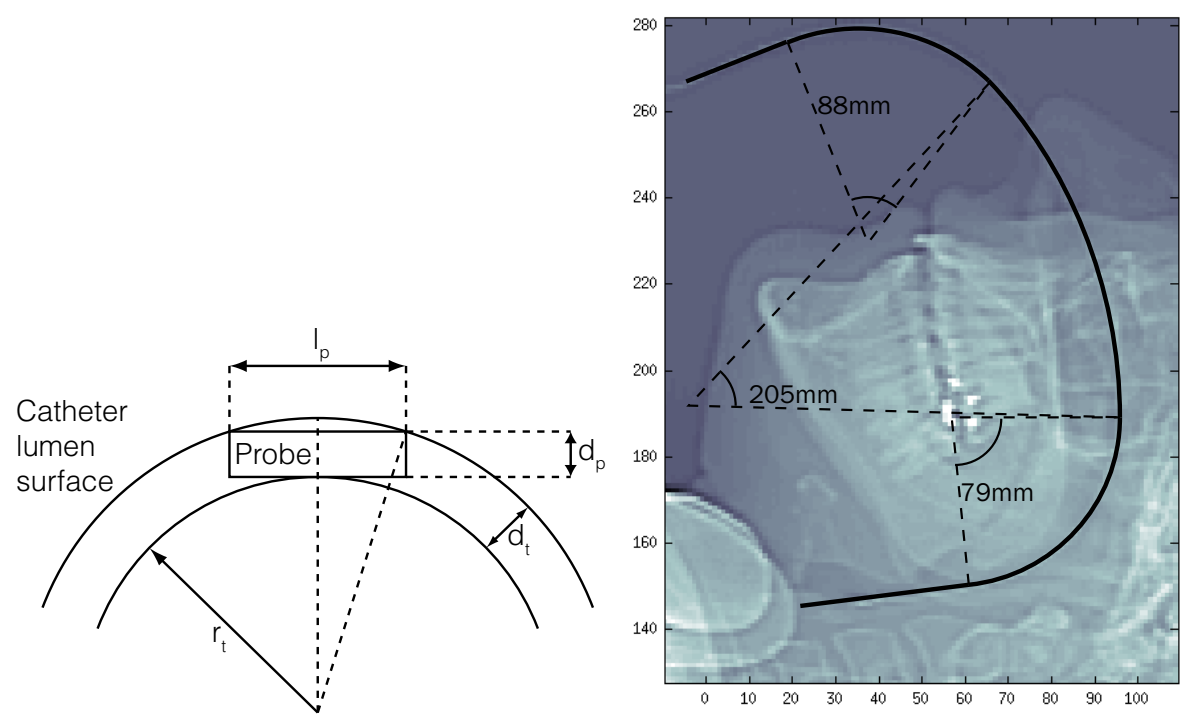

Figure 4.9: (a) Geometry used to calculate the minimum bend radius for a probe and catheter characteristics. (b) CT image of an adult with a catheter and probe in the upper airway. The catheter path is overlaid with circular segments showing the radius of curvature for each segment.

tector circuit (AD606JR) [129], as shown in Figure 4.10. Electronic envelope detection reduces the processing overhead of the software and logarithmic encoding is convenient for achieving a large system dynamic range. The output envelope signal is then lowpass filtered and level shifted before being digitized by a $5 \mathrm{MS} / \mathrm{s}$ 12-bit data acquisition card (National Instruments); Figure 4.11 shows a typical digitised A-scan. The bandpass and lowpass filters were designed in the form of plug-in modules which could be interchanged for different scanning speeds. The bandpass filter, a passive, second-order resonant LC circuit, had a bandwidth set for optimum SNR as discussed in Section 3.4.6. Similarly, the lowpass filter bandwidth was set to half of the bandpass filter bandwidth to maximize SNR.

\subsection{Sensitivity and intensity calibration}

The reflectivity sensitivity of the $a \mathrm{OCT}$ system is determined by measuring the SNR of a calibrated reflector $\left(R_{\text {test }}\right)$ in the sample arm. As the signal power is proportional to the sample reflectivity, the sensitivity is then given by

$$
S_{\text {system }}[\mathrm{dB}]=S N R_{\text {test }}[\mathrm{dB}]-R_{\text {test }}[\mathrm{dB}]
$$




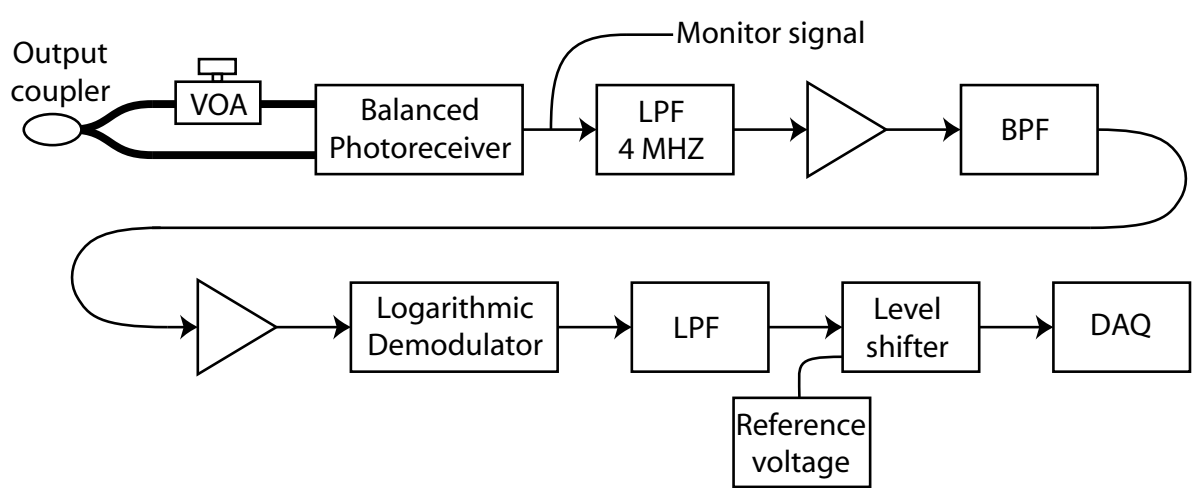

Figure 4.10: Schematic diagram of the signal processing electronics. VOA, variable optical attenuator; LPF, lowpass filter; BPF, bandpass filter; DAQ, data acquisition card in the personal computer.

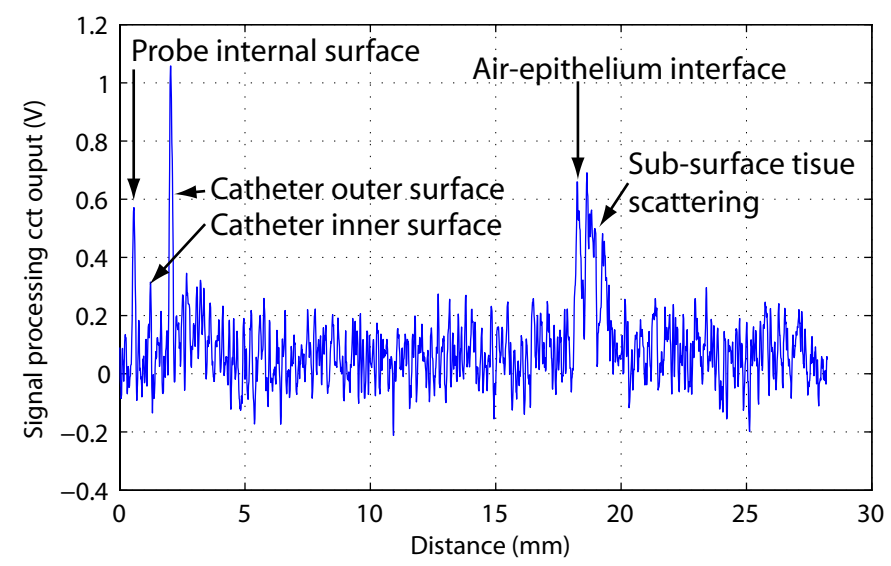

Figure 4.11: An example of typical data from a single A-scan. Reflections from the probe, catheter surfaces and air-tissue interface are labelled.

Figure 4.12 shows a measurement made to determine system sensitivity. A known sample reflectivity of $-56.2 \mathrm{~dB}$ is created by an attenuated reflection from a microscope slide. The measured SNR is $41.7 \mathrm{~dB}$, giving a sensitivity of $97.9 \mathrm{~dB}$. The dominating noise factor is presently beat noise from mixing between the reference light and incoherent light reflected from the fibre-optic rotary joint. The predicted sensitivity using the model discussed in Section 3.4.2 is $-102 \mathrm{~dB}$. The difference between predicted and measured values could be explained by extra noise added by the signal processing circuitry, non-ideal filters, and uncertainty in the sample reflection value. 


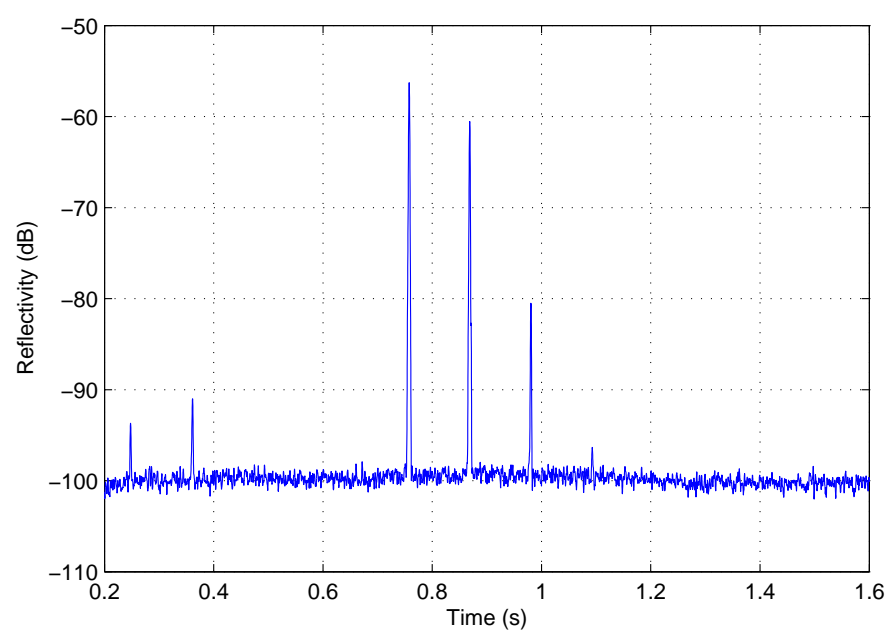

Figure 4.12: Example of sensitivity calibration measurement. The reference of $-56.2 \mathrm{~dB}$ is created using a microscope slide reflection with attenuation.

\subsection{Distance calibration}

The OCT distance ranging is calibrated by translating a surface to a series of known positions (using a 5-micron accuracy vernier micrometer) while recording the OCT signal (Figure 4.13). The probe is held fixed and the translation stage aligned so that it moves perpendicularly to the beam. The surface used is a microscope slide which is aligned such that specular reflections are not captured (only scattered light). The positions of each surface in the OCT data are determined automatically by a Matlab script which finds the average peak position for each of the stationary periods (minimum 10 seconds). A linear fit between the OCT signal peak positions and the actual physical positions is performed and used to calculate the calibration factor. The factor is then entered into the $a \mathrm{OCT}$ real-time software interface.

\subsection{Probe control}

The sample arm was connected to the interferometer using a fibre-optic rotary joint (Focal Technologies, Nova Scotia, Canada), or FORJ, which allowed the probe to be rotated freely. The rotation was driven by a linear $12 \mathrm{~V}$ DC motor under microprocessor control, see Figure 4.14 for a 3D model of the subsystem. The drive system had a planetary gearhead which was interchanged when different speed ranges needed to be accessed. For typical operation, a 100:1 gearhead allowed the probe to be driven from $0.2 \mathrm{~Hz}$ to $1.25 \mathrm{~Hz}$. The FORJ had a maximum return loss of -41.2 (tested during rotation of $100 \mathrm{rpm}$ for 5 minutes), and an in- 

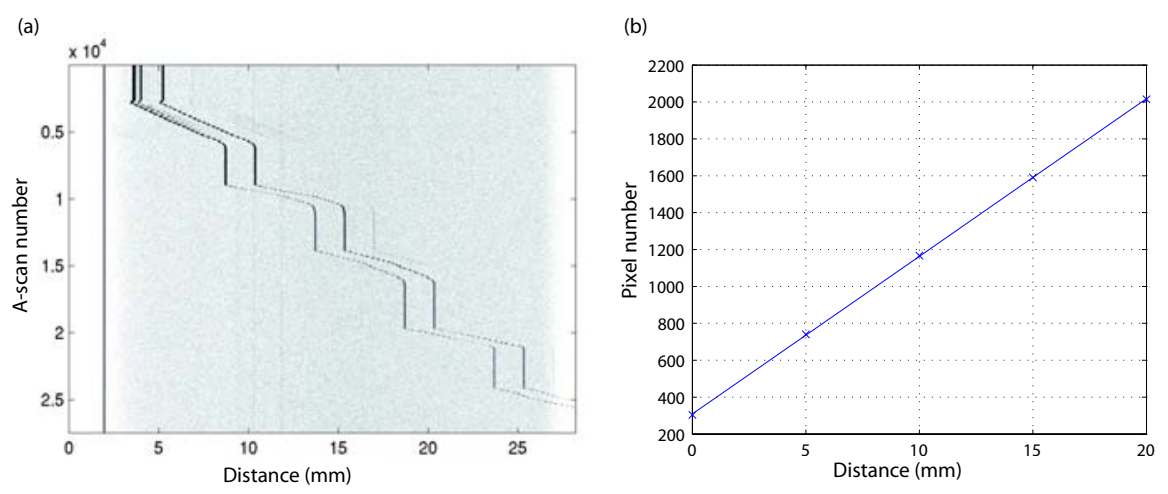

Figure 4.13: Example of distance calibration measurement. (a) Raw data from a reflector moved to a series of known positions, (b) Fitted line for checking the scan linearity and calculation of the distance calibration factor.

sertion loss which varied from 1.6 to $2.0 \mathrm{~dB}$ over each rotation. The variation is caused by minute misalignments in the assembly which affect the coupling efficiency between the two single-mode fibres in the FORJ (one rotating and one stationary).

The motor-drive and FORJ assembly was mounted on a ball-screw carriage so the probe could be translated forward or back (in and out of the catheter). The ball-screw was driven by a $12 \mathrm{~V}$ stepper motor. A microprocessor control unit (Atmel AT90S8515) controlled the two motors and recorded digital pulses from an angle encoder connected to the FORJ. The microprocessor maintained accurate angular and translational position information, and had a small control and interrogation instruction set. The $a \mathrm{OCT}$ software on the personal computer communicated with the microprocessor via a RS232 serial link. For convenience, the microprocessor could also be controlled from buttons on the front panel of the FORJ/microprocessor housing. For safety there was also a cut-off switch to manually interrupt motor power.

The FORJ had a specification of $-0.5 \mathrm{~dB}$ variation in insertion loss over a full rotation. This amount of loss is virtually indetectable against the natural variation of optical loss due to differences in optical power reflected by the tissue and collected by the micro-optics as the probe rotates.

\subsection{Integration with clinical data recorders}

One of the aims of the $a \mathrm{OCT}$ development was integration with other equipment used in sleep clinics, such as a polysomnography suite. Polysomnography involves the measurement an array of parameters such as EEG, ECG, EMG, 


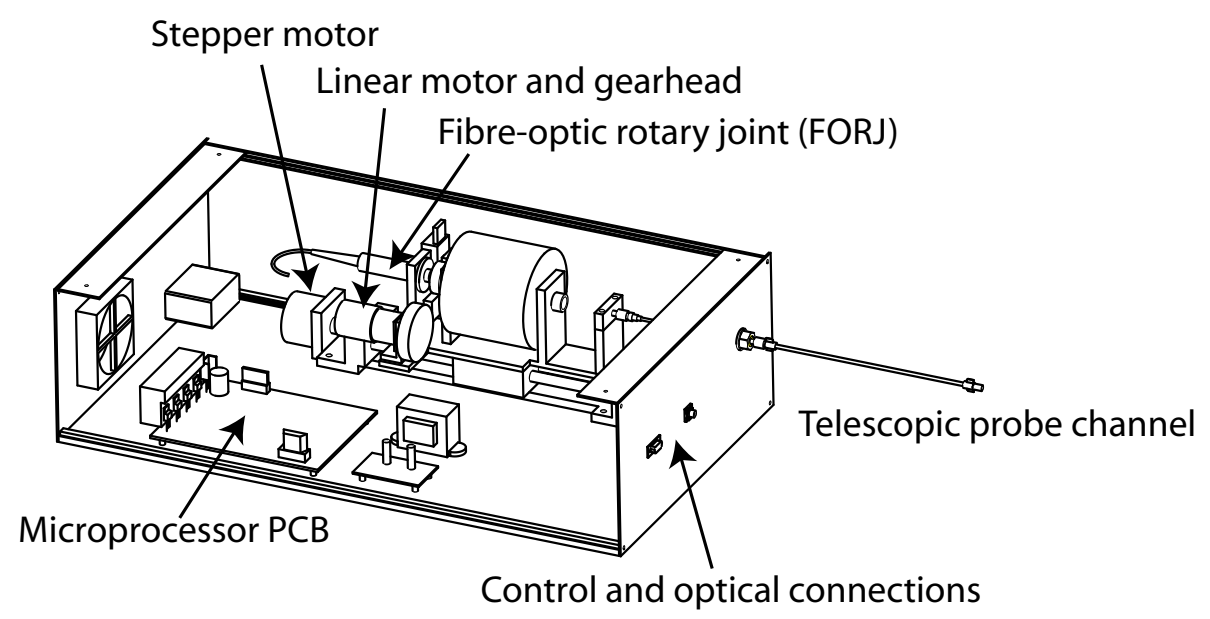

Figure 4.14: CAD model of the probe control subsystem. The main functions are the rotation and translation of the probe, and position reporting.

$\mathrm{SaO} 2$, audio, video signals [130]. These signals are digitized and saved using central signal management software, and then are available for later processing and playback. Powerlab (ADInstruments, Colorado Springs, CO, USA) and ProFusion (Compumedics, Abbotsford, VIC, Australia) are two such systems which we have used for experiments in the sleep clinic (Sir Charles Gairdner Hospital, Western Australia).

To integrate the aOCT system into the clinical session the time synchronization needed to be managed to ensure that the data could be accurately correlated in later analysis. It would be possible to augment the aOCT system to capture other analogue and digital signals at the same time as the OCT signal, however, the limit on the number of possible inputs would inevitably restrict future experiments. The approach taken to avoid this restriction was to instead synchronize the $a \mathrm{OCT}$ system and the exisiting laboratory data loggers. This was done by creating synchronization output channels on the aOCT system which are recorded on the other systems. The synchronization channel performs two functions, (a) provision of a local time datum for each experiment session, and (b) the provision of a continuous clock signal to maintain synchronization once established. The time datum is necessary because personal computers using simple quartz oscillators circuits do not maintain an accurate clock and would disagree by several minutes after a day or two of free running. The continuous clock signal is used to correct sampling clock skew between systems during the experiment.

Figure 4.15 illustrates the synchronization channels generated by the aOCT system by TTL logic outputs of the data acquisition card. These are recorded by 
other laboratory data recorders at a sampling rate high enough to clearly distinguish the edge transitions. The first channel contains a series of pulses encoding of a unique string using Manchester-encoded digital modulation. The unique string is generated by using the date and time of the experiment commencement and serves as marking the experiment datum. The second channel contains a clock signal at the OCT A-scan rate that is used for clock skew compensation. By this method the level of differential clock error was improved from $2000 \mathrm{~ms}$ (a maximum error observed) to $<33 \mathrm{~ms}$.
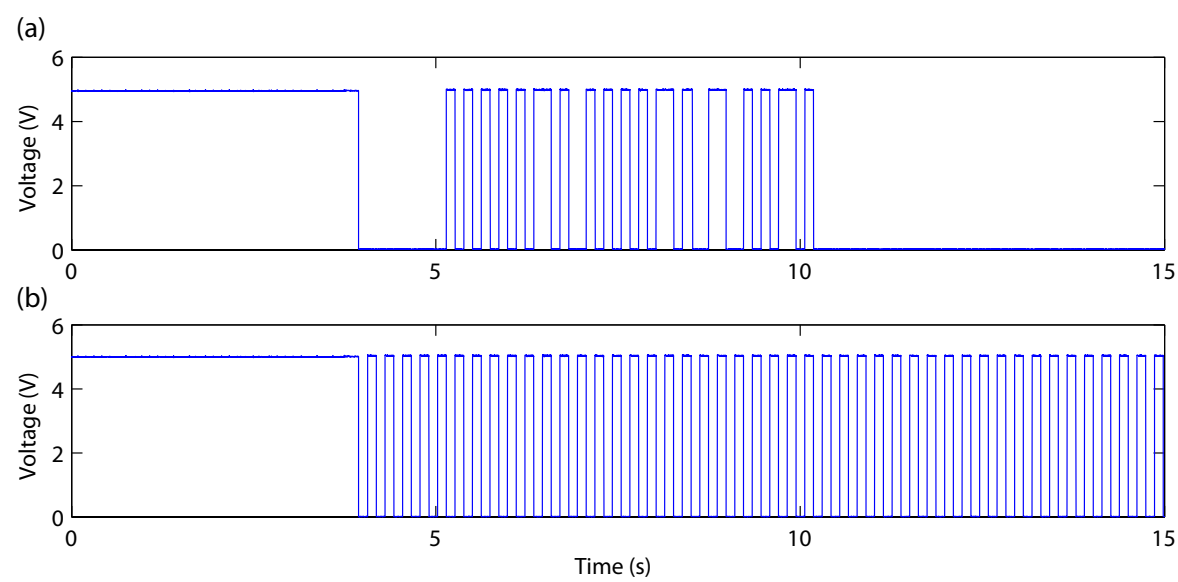

Figure 4.15: The synchronization outputs from the aOCT system. These outputs are used to facilitate automatic synchronization of data recorded on different laboratory sensor data acquisition systems. The upper plot shows the first channel, in which the edge of the first transition is used as the datum, and the series of pulses following it are a unique identification code. The lower plot shows the second channel, which contains a clock signal for clock skew compensation.

\subsection{Software}

The aOCT system was controlled by a Microsoft Windows C++ application. The software used a multi-threaded architecture in order to manage its functions of acquiring data, saving to disk, processing and displaying data, and controlling the system (Figure 4.16). A multi-threaded software model was used to cope with the high data rate $(162 \mathrm{MB} / \mathrm{min})$, as the program components have different and variable requirements for access to the computer resources. A circular buffer, with first-in first-out access, was used to manage the data, with the 'save' thread given priority access to the raw data. A similar programming approach in OCT software has also been published [131]. Address pointers were used to keep track of the current insertion point in the data stack and the read locations 
of the save and display threads. For reliability, the lagging of the display thread would trigger a warning notice and interruption of data display to allow the save thread to continue. Figure 4.17 shows the interface of the aOCT software, which was designed to be easy and efficient to use in a clinical setting.

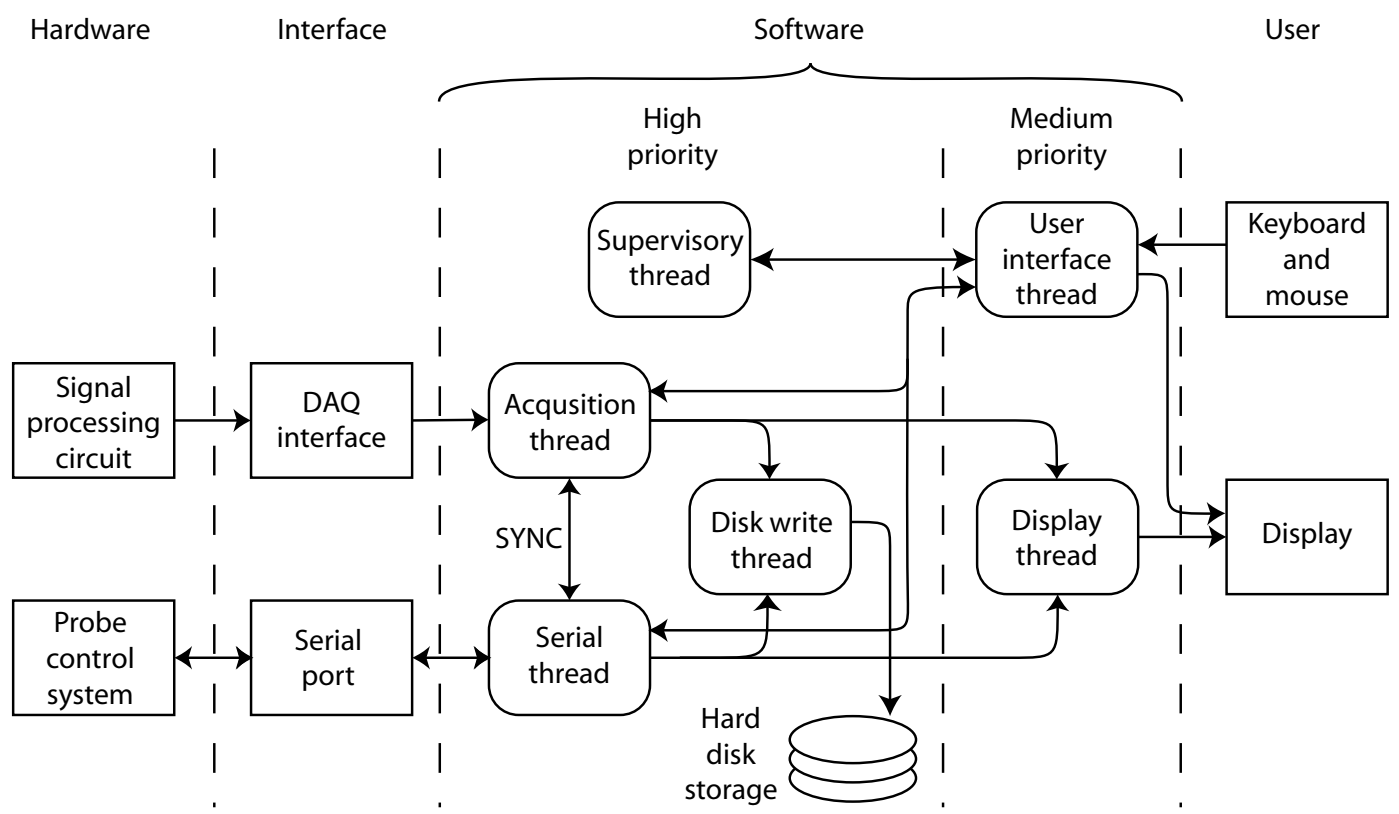

Figure 4.16: Schematic of the $a \mathrm{OCT}$ software.

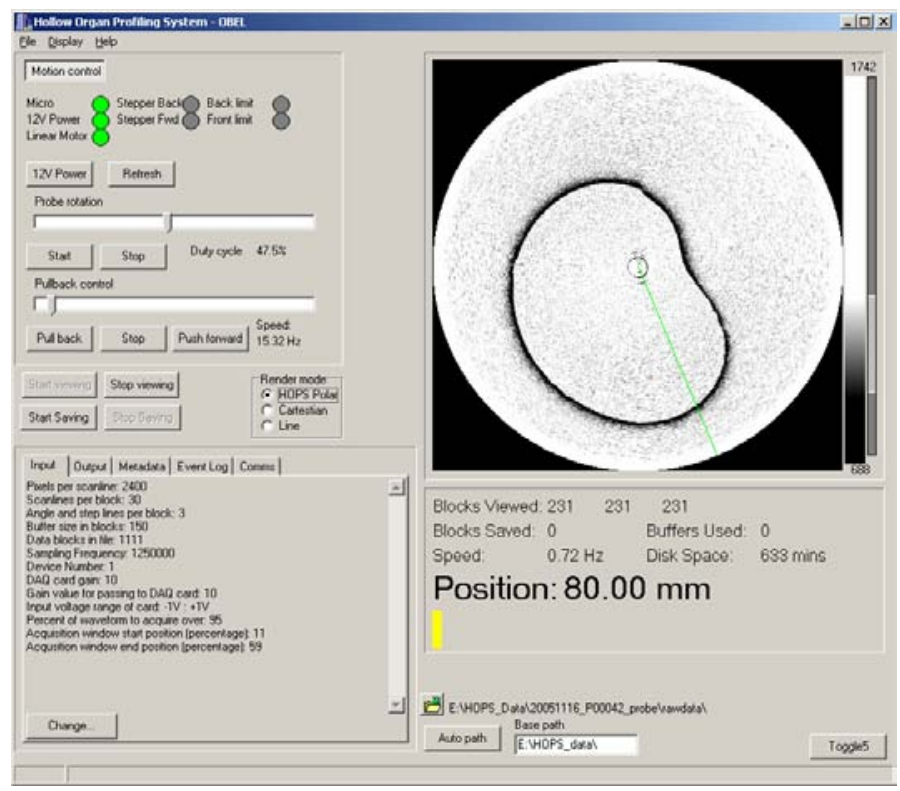

Figure 4.17: Front panel screenshot

Given the resource-intensive data acquisition, saving, and display operations, it was necessary to use an efficient algorithm for the real-time data display. The 
most CPU-intensive operation was the conversion of the data from a polar basis to a Cartesian basis (Figure 4.18). The algorithm had to update the display as the data was collected in discrete blocks by the data acquisition card. Each block consisted of a set of 30 A-scans, each containing 5800 pixels. The steps in the algorithm are: (a) Find the area of the display to be updated. This is determined by calculating the screen coordinates of the segment to be drawn based on the microprocessor probe angle information corresponding to a particular block of data. (b) A lookup table is used to convert the screen coordinates $(x, y)$ of each pixel in the segment to physical coordinates (angle, range), and finally data coordinates (row, column). Lookup table operations were used because they are faster than trigonometric calculations. (c) The value in memory at the data coordinates is then used to calculate a screen pixel value to be copied to the screen image buffer. The pixel colour and brightness were determined by a dynamic range transformation and colour-map lookup. The display dynamic range settings are important as computer displays typically have an 8 bit resolution in intensity level (i.e. 256 distinct levels), whereas the $a \mathrm{OCT}$ data has a higher dynamic range of 12 bits (limited by the data acquisition card). In medical systems typically the dynamic range transformation is linear and the two mapping parameters are known as the 'level' and 'window' [132]; this approach was used here. The level and window parameters could be controlled interactively with a graphical control on the software interface in order to maximise the contrast in the real-time $a \mathrm{OCT}$ image between the airway and the background noise.

In addition to saving $a$ OCT signal data, the software also recorded the probe angle and pullback position data, timestamp data for synchronization purposes, event logs, and a metadata structure containing a description of the experiment. The event logs were generated during the experiment and were used to record descriptions and the times of events which were important for the later analysis of the data. For example, the event of an involuntary swallow by a subject, which markedly affects the pharynx shape and dimensions for a few seconds, usually occurred at least once during a pullback scan. The function keys on the keyboard were used to trigger events, and each key could be preset with an event description, such as "mandible advancement 100\%" (settings could be saved as sets of events for different experiment types). The metadata structure recorded the subject details (subject ID, head, body position) and experiment conditions, and was permanently associated with the $a \mathrm{OCT}$ data. 
(a)

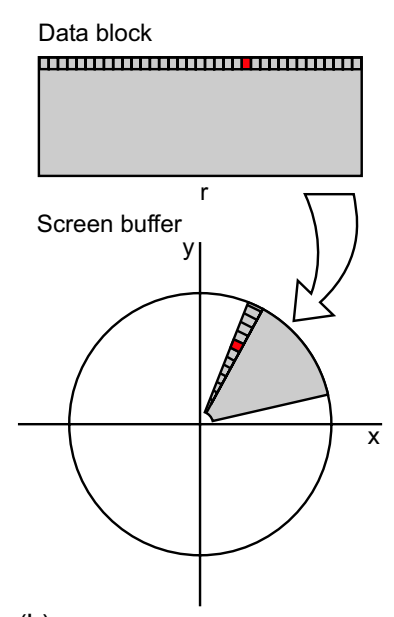

(b)

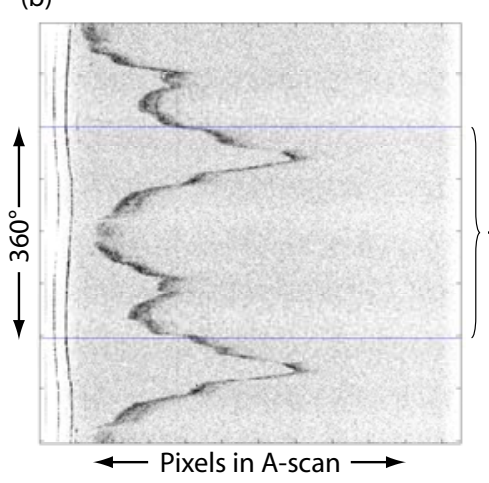

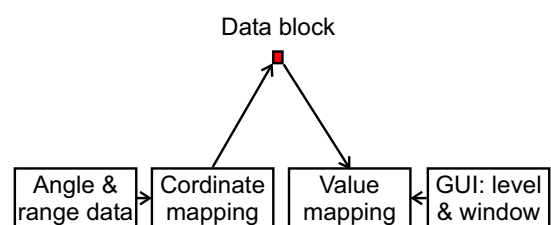

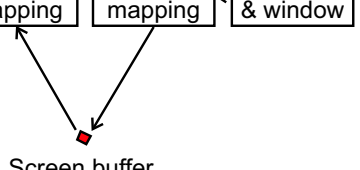

Screen buffer

(c)

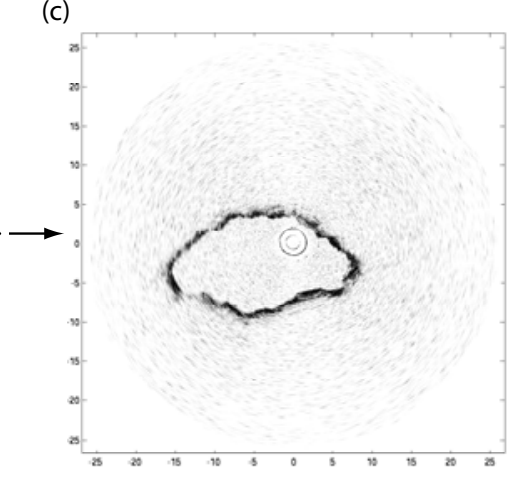

Figure 4.18: (a) Process of rendering of polar-encoded data to Cartesianbased display field, (b) example of raw digitized data, (c) one frame of the data displayed in polar form.

\subsection{Lateral resolution of the HOPS images}

The lateral resolution at any particular point in the aOCT images is defined as the width of the point reflector in the direction perpendicular to the OCT a-scan (which is in the radial direction). This resolution is limited by two parameters, (a) the size of the beam, and (b) the rate of sampling in the transverse direction. The size of the beam is determined by the properties of the beam produced by the optical probe, and can be modelled using Gaussian beam optics as discussed in Section 4.2.2. The rate of sampling in the transverse direction, $R_{\mathrm{tx}}$ in A-scans per radian, can be calculated from

$$
R_{\mathrm{tx}}=2 \pi f_{\mathrm{rot}} \Gamma
$$

where $f_{\text {rot }}$ is the A-scan repetition rate $(\mathrm{Hz})$ and $\Gamma$ is the probe rotation rate $(\mathrm{Hz})$. Then the spacing between samples in the transverse direction can is calculated 
using

$$
\Delta x(r)=\frac{r}{R_{\mathrm{tx}}}
$$

where $r$ is the distance from the probe. If $\Delta x(r)$ is less than the beam size then the aOCT image resolution will be degraded by undersampling in the tranvserse resolution. This is not necessarily a problem, especially if the aOCT image is being used mainly for location of the airway walls. However, if the subsurface information or scattering properties of the surface is of interest, it would be best to avoid undersampling in this case. This could be achieved by either reducing rotation rate, increading the OCT A-scan rate, or reducing the distance from the probe which can be imaged. Figure 4.19 shows the transverse resolution based on the beam size calculation and A-scan spacing, for parameters based on typical usage of the $a \mathrm{OCT}$ system for measurements in the upper airway. The undersampling is worst at the periphery of the images, where the A-scans are spaced by almost $0.8 \mathrm{~mm}$ while the beam size is slightly more than $0.2 \mathrm{~mm}$.

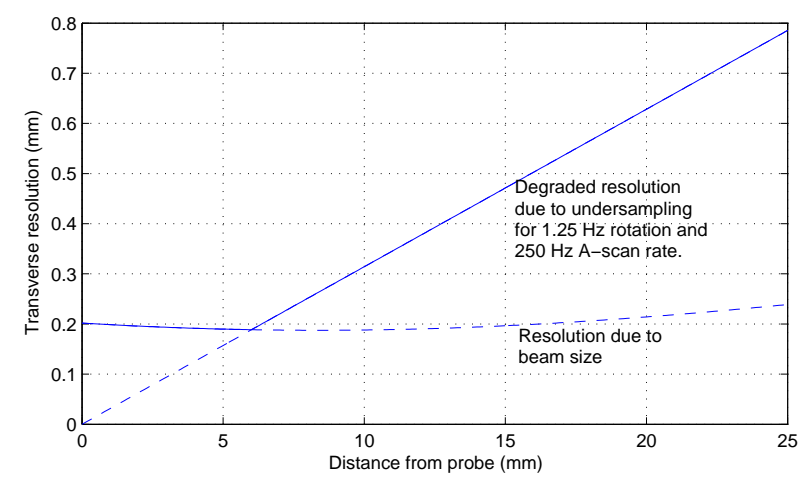

Figure 4.19: Transverse resolution (solid line) of the HOPS images due to the combination of beam width (blue dashed) and angular sampling rate (red dashed).

\subsection{Data processing and presentation}

Offline data processing was done in Matlab. Typically the data was first converted from polar coordinate form to Cartesian coordinates for display. The Matlab scripts used an interpolating renderer to transform the polar coordinate data to a Cartesian basis, rather than the nearest neighbour approach of the realtime software which was computionally cheaper. Subsequently, image enhancement, analysis, 3D reconstruction, etc. could be carried out. Scripts were written to extract longitudinal sections and synchronize the aOCT data with the data from other sources, ProFusion or PowerLab. 


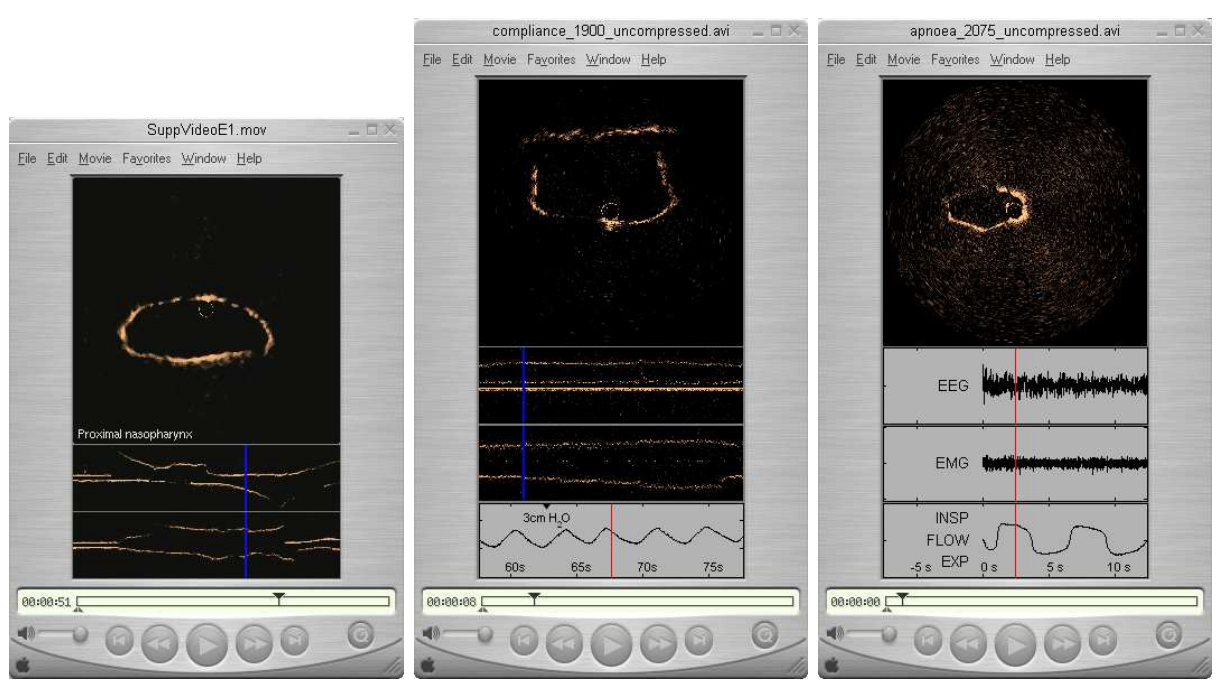

Figure 4.20: Screenshots of typical video files used for later review of data by the clinician. Shown are, from left to right, a measurement of the whole pharynx, an airway compliance experiment, and a measurement taken during validated sleep.

The Matlab scripts generated movie files which were compressed using MPEG or Sorenson Video 3 codecs. Figure 4.20 shows screenshots of the video rendering of a pullback. Images were filtered before video compression to reduce the level of artefacts created by the compression (this was particularly necessary for MPEG compression). Median filtering with a $3 \times 3$ matrix was found to be effective for this.

4.10.1 Contour location The primary information contained in each crosssectional $a \mathrm{OCT}$ image is the location of the air-tissue interface around the circumference of the airway lumen. This data can be represented as a one dimensional function of radius versus angle. The problem of reducing the 2D image data down to a 1D description based on regions and boundaries is known as contour extraction and is a wide and highly active field of research. There is a wide array of manual, semi-automatic and automatic approaches depending on the particular characteristics of the data and application. $a \mathrm{OCT}$ images present a difficult problem for contour location techniques because of the large variability in the images. It is not possible to know beforehand how many discrete edges there are or where they will be in the image. The most significant a priori information is that there should only be one airway-lumen boundary detected on each radial scanline, and that there will always be a circular contour in the centre of the image which is the catheter surface. Four of the most popular edge contour location 
technique categories are listed here with a discussion of the applicability of each to $a$ OCT images.

Level-based segmentation and region growing methods: A common approach to medical image segmentation is the use of intensity thresholds based on intensity histogram shape analysis. Some imaging modalities produce bimodal or multimodal intensity value distributions - typically the background pixels and the objects of interests form the histogram peaks. This method is not readily applicable to $a \mathrm{OCT}$ images as only boundaries of the airway lumen create a signal contrast.

Curve fitting methods: These are suitable when the approximate shape of the curve is known a priori. While some characteristics of the aOCT images are known, there is a large variability in edge contrast and even the topology of the detected airway lumen. This precludes the use of simple curve fitting techniques.

\section{Edge tracking and linking methods}

Edge linking techniques have been used in OCT image processing. Edge linking has been used to accurately locate the boundary between cartilage and bone in subsurface OCT images [133]. Prefiltering was carried out to reduce the level of speckle (RKT transform filter [134]) and to enhance the edges (Sobel edge detection). In this situation, however, there is quite a high level of a priori information about the existence of a boundary and the approximate location. An approach using a Markov boundary model was used for locating boundaries in retinal OCT images [135], however, the structure of the images was also quite constrained in this application.

\section{Active contour methods}

A useful technique for contour location when the contour is a single closed curve is the use of active contours, also known as "snakes" [136]. Snakes locate a contour by iterating from an initial guess contour by iterative deformations which act to minimize energy computed using an energy function. This function contains internal and external energy terms. Internal energy refers to energy calculated from the contour itself, such as tension and resistance to bending. External energy is calculated from the image pixel values. In an improved version of the active contour algorithm, the external energy is calculated instead from a gradient vector flow (GVF) map calculated from the original image, which allows a much greater range of initial contours that successfully converge on the solution [137]. A limitation of this kind of contour is that it assumes that the lumen will be a single closed curve, however, this is frequently not the case. An example 
is the oropharynx, where the mouth opening to the pharynx causes a gap in the airway contour. Another is the situation where part of the airway shadows the beam, such as in the hypopharynx, where the epiglottis can block the beam from reaching the anterior airway surface.

From the above techniques, the edge tracking and active contour methods were chosen for further investigation of their usefulness in extracting the airway lumen profile. The edge tracking method was implemented using a "virtual robot approach" [138] (this work was carried out with Thomas Gutzler, an intern student). The software was written in IDL (Research Systems, Inc., CO, USA). In this method an initial point is provided by a human operator and the algorithm then moves the point along the boundary, tracing out the profile as it goes. From the initial point the algorithm chooses at random one of the two possible directions to commence travel along the boundary. The algorithm then determines the direction of highest intensity pixel in a circle of a certain radius around the point. The radius is reduced in steps to 1 pixel, at each step searching for a pixel of higher intensity. The algorithm also has a weighting factor favouring motion in the similar direction to the last step, this prevents the robot from back-tracking. By using the search over a variable radius circle the robot can "jump" gaps in the profile. The various parameters in the algorithm determine the tightness of bends the robot can make, the size of gaps that can be jumped, and the extent to which the robot resists suddent changes in direction. Figure 4.21 shows an example of the algorithm successfully applied to a selected $a$ OCT profile of an irregular paper tube (paper is strongly scattering and provides a high SNR). However, in general, the virtual robot approach was not successful in consistently following boundaries which were weakly defined or had large gaps. Also the presence of speckle strongly affected the result as the robot would start following a collection of random speckles rather than the actual airway lumen.

Of the various active contour techniques the GVF active contour method based on work by Xu et al. [137] has shown the most promise. Figure 4.22 shows a preliminary trial of GVF snakes showing a contour accurately finding the airway lumen from an initial contour placed around the image boundary (this work was carried out with Jonathon $\mathrm{Ng}$, a final year project student). The algorithm was implemented in Matlab. Median filtering was used to reduce the level of speckle in the images before application of the GVF snakes algorithm. This work is still in progress and is not yet advanced sufficiently to be automatically applied to many aOCT images. The main outstanding problem is the poor handling of weak signal 

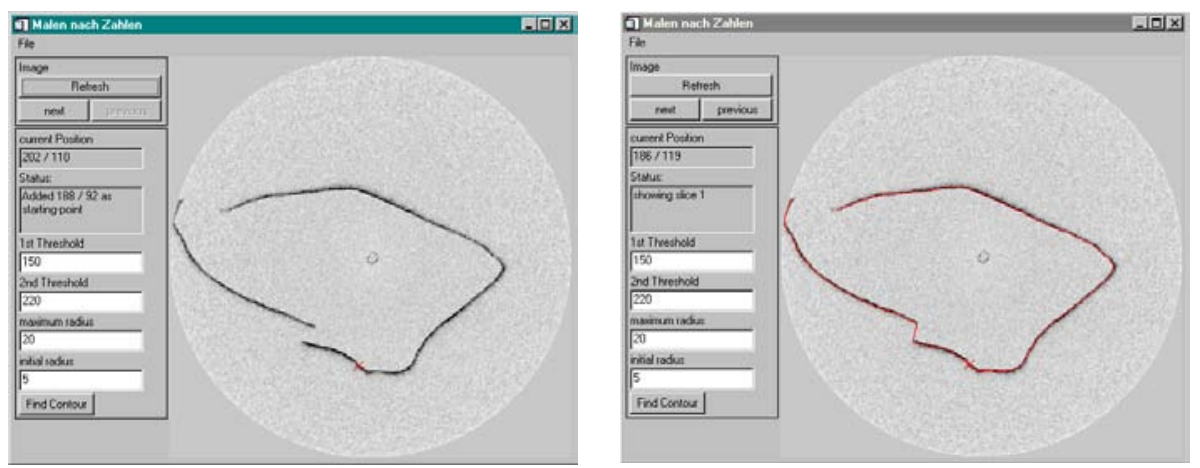

Figure 4.21: Example of contour successfully drawn using the virtual robot approach. The paper tube model gave a strong boundary signal for the algorithm to lock onto.

levels in the presence of speckle, or gaps in the airway profile due to the actual anatomy. In these cases the algorithm could give very unpredictable results and require the intervention of a human operator.

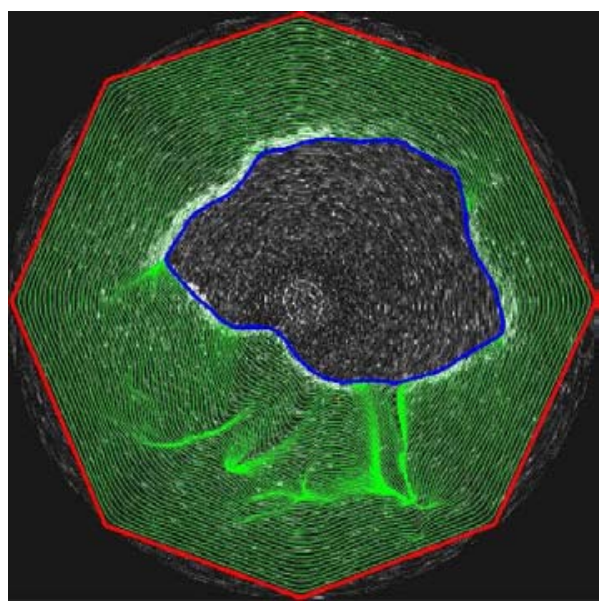

Figure 4.22: Example of GVF active contours converging on an airway boundary.

4.10.2 Three-dimensional reconstruction As a pullback experiment generates a three-dimensional data set it would be convenient to display the data as a single 3D model. This would enable visualization of the entire hollow organ anatomy, a useful adjunct to cross-sectional area and dimensional measurements. Although the translational movement of the $a \mathrm{OCT}$ probe is currently too slow to capture rapid changes in volume, such as an apnoea collapse, with a speed increase it may be possible to quantitatively monitor volume changes. Such measurements will permit calculation of segmental compliance (volume vs pressure) and allow comparison of upper airway structure and function between normal 
and apneic subjects at a level of complexity and comprehensiveness that cannot be obtained from two-dimensional images. To create a 3D model using Matlab, the data was assembled into two-dimensional slices and vector contours were extracted. These were then used to generate a stack of thin shells rendered in a Matlab plot window. Figure 4.23 shows an example created from a $20 \mathrm{~mm}$ segment of a pullback which includes the oesophagus and hypopharynx.

The 3D models were also converted to Quicktime VR files allowing interactive manipulation of the 3D model with the standard Quicktime Player application. In the future this may be useful for visualization of the airway, in the same way that interactive three-dimensional models have been used for "virtual endoscopy" in other parts of the body [139-141].

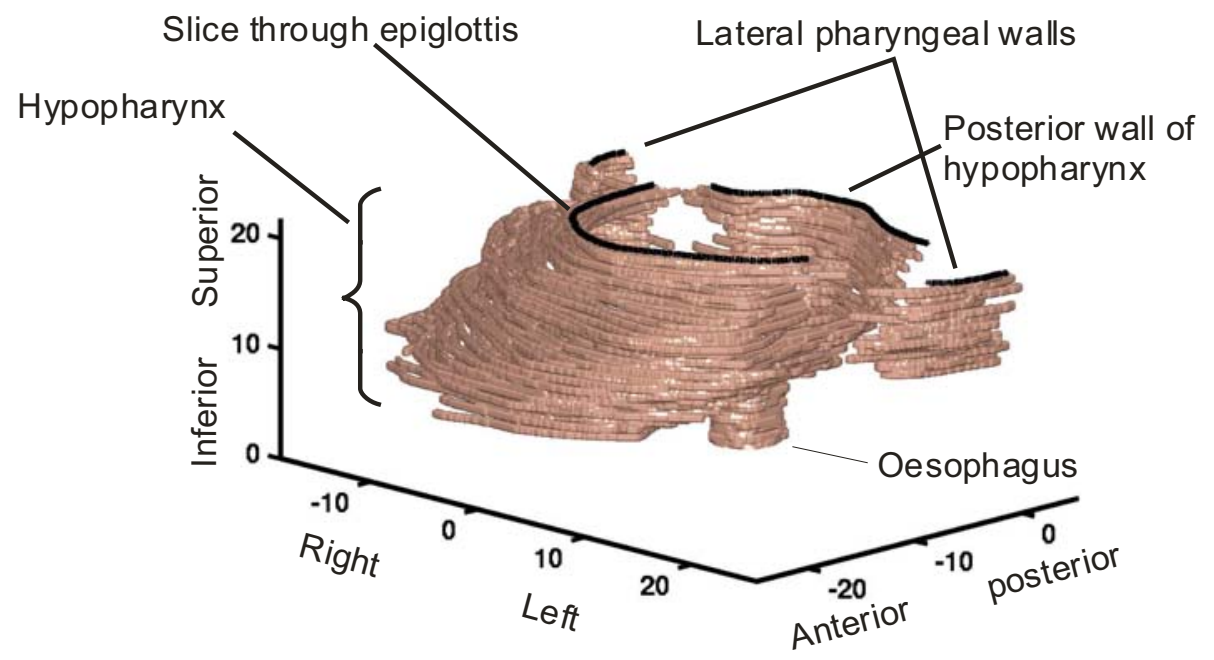

Figure 4.23: Three-dimensional model created from a $20 \mathrm{~mm}$ segment of a pullback data set.

\subsection{Summary}

This chapter presented development and a technical description of the first prototpye $a \mathrm{OCT}$ system as implemented, with attention paid to the engineering details which are unique to this instrument. In particular, optical and mechanical aspects of the sample arm, and the details of the software and data processing, were areas which required significant design. This chapter has not emphasised actual results and measurements taken using the system; the next chapter in this 
thesis is a published paper in a medical journal containing examples of these results. 


\section{Clinical application}

This chapter consists of a paper presenting the initial clinical possibilities of the aOCT system. It was published in the American Journal of Respiratory and Critical Care Medicine, vol. 173, pp. 226-233, 2006. There are four online supplement files, one document (included here) containing extra details about the experimental methods and exanded statistics tables, and three video files of $a$ OCT data. The paper and supplementary files can also be found online at:

http://ajrccm.atsjournals.org/cgi/content/abstract/173/2/226 and also at the OBEL research group publications webpage:

http://obel.ee.uwa.edu.au/publications/index.html

All citations in this chapter are listed in the chapter bibliography rather than the thesis bibliography.

\subsection{Quantitative upper airway imaging with anatomical optical coherence tomography}

Julian J. Armstrong1 ${ }^{1}$, Matthew S. Leigh ${ }^{1}$, David D. Sampson ${ }^{1}$, Jennifer H. Walsh ${ }^{2}$, David R. Hillman ${ }^{2}$, Peter R. Eastwood ${ }^{2,3}$

${ }^{1}$ Optical+Biomedical Engineering Laboratory, School of Electrical, Electronic and Computer Engineering, University of Western Australia, Crawley, Western Australia 6009, Australia

2 West Australian Sleep Disorders Research Institute, Department of Pulmonary Physiology, Sir Charles Gairdner Hospital, Nedlands, Western Australia 6009, Australia

${ }^{3}$ School of Anatomy \& Human Biology, University of Western Australia, Crawley, Western Australia 6009, Australia

\section{CONFLICT OF INTEREST STATEMENT}

The authors declare they have no competing financial interests.

This article has an online data supplement. 
Subject Category Number: 110

We acknowledge the early support for this work from the Medical Research Foundation of Western Australia and the Australian Health Management Group and more recent support from the National Health and Medical Research Council (Australia) (Development Grant ID No. 303319). These funding contributors had no role in study design, data collection, data analysis, data interpretation, or writing of the report. PRE is supported by a National Health and Medical Research Council (Australia) R Douglas Wright Fellowship (No. 294404).

\subsection{Abstract}

Background: Measurements of upper airway size and shape are important in investigating the pathophysiology of obstructive sleep apnea (OSA) and in devising, applying and determining the effectiveness of treatment modalities. We describe an endoscopic optical technique (anatomical optical coherence tomography, $a \mathrm{OCT}$ ) that provides quantitative real-time imaging of the internal anatomy of the human upper airway.

Methods: Validation studies were performed by comparing $a \mathrm{OCT}$ - and CT-derived measurements of cross-sectional area (CSA) in: (i) conduits in a wax phantom; and (ii) the velo-, oro- and hypopharynx during wakefulness in 5 volunteers. aOCT scanning was performed during sleep in one subject with OSA.

Results: aOCT generated images of pharyngeal shape, and measurements of CSA and internal dimensions that were comparable to radiographic CT images. The mean difference between $a \mathrm{OCT}$ - and CT-derived measurements of CSA in (i) the wax phantom was $2.1 \mathrm{~mm}^{2}$ with limits of agreement (2SD) from -13.2 to $17.4 \mathrm{~mm}^{2}$ and intra-class correlation coefficient of 0.99 ( $\mathrm{p}<0.001$ ), and (ii) the pharyngeal airway was $14.1 \mathrm{~mm}^{2}$ with limits of agreement from -43.7 to $57.8 \mathrm{~mm}^{2}$ and intra-class correlation coefficient of 0.89 ( $\mathrm{p}<0.001$ ). aOCT generated quantitative images of changes in upper airway size and shape before, during and after an apneic event in an individual with OSA.

Conclusions: aOCT generates quantitative, real-time measurements of upper airway size and shape with minimal invasiveness, allowing study over lengthy periods during both sleep and wakefulness. These features should make it useful for study of upper airway behavior to investigate OSA pathophysiology and aid clinical management. 


\section{KEYWORDS}

upper airway anatomy, optical coherence tomography, sleep apnea

\section{WORD COUNT}

Abstract: 249 words

This article has an online supplement, which is accessible from this issue's table of contents at www.atsjournals.org

\subsection{Introduction}

Measurements of upper airway size and shape are important in investigating the pathophysiology of obstructive sleep apnea (OSA) and in devising, applying and determining the effectiveness of treatment modalities [1]. However, a persisting limitation is the absence of an imaging technique that satisfactorily provides safe, accurate and repetitive measurements of upper airway dimensions during wakefulness and sleep. Radiographic computed tomography (CT) and fluoroscopy involve potentially hazardous ionizing radiation. Magnetic resonance imaging (MRI) is expensive, noisy, claustrophobic and incompatible with metallic probes and catheters. Neither modality is suitable for lengthy evaluations or evaluations during sleep [2]. Ultrasound is not feasible because of poor transducer-air coupling, an unavoidable problem in air-filled organs. Fiber-optic nasoendoscopy has been used to visually examine the anatomical features and structure of the airway but its quantification of airway dimensions is indirect and subjective [3]. Measurement of airway calibre using acoustic reflection is not possible distal to the site of collapse, as the acoustic waves will not penetrate a collapsed airway [4].

In this paper, we describe an endoscopic optical technique that generates quantitative, real-time images of the upper airway that enable accurate determination of shape and size. Briefly, an optical probe is placed inside a catheter, which is inserted via the nares to the level of the mid-esophagus. Rotation of the probe within the catheter provides a 360 degree profile of surrounding tissue. The optical probe can be systematically moved within the catheter, allowing the upper airway to be scanned at multiple sites without stimulating the airway mucosa.

The technique is adapted from optical coherence tomography (OCT) [5], a medical imaging modality. OCT is an optical sectioning microscopy modality which has been used to image subsurface tissue morphology in fields including 
ophthalmology [6], dermatology [7], vascular medicine [8], gastroenterology [9], and urology [10]. Our adaptation involves a substantial increase in its axial distance range to allow macroscopic imaging of upper airway internal anatomy. We refer to this adaptation as 'anatomical OCT' (aOCT) to distinguish it from the existing microscopic, subsurface OCT techniques[11].

This paper presents the operational capabilities of the system, including data regarding its validity and applicability to quantitative measurement of human upper airway size and shape.

\subsection{Methods}

Baseline in vitro validation studies were performed by comparing $a \mathrm{OCT}$-derived measurements of cross-sectional area (CSA) with those derived from (i) circular holes of known size in a wooden template, and (ii) CT-derived measurements of holes with varying shape and size cut in a wax phantom. Following these measurements, in vivo studies were performed to (i) compare aOCTderived measurements of upper airway shape and size at the levels of the velo-, oro-, and hypopharynx with those obtained from simultaneous CT scans, and (ii) determine the reproducibility, and intra- and inter-observer variability of $a$ OCTderived measurements of upper airway shape and size. Additional in vivo measurements were performed to demonstrate the feasibility of undertaking such measurements to determine pharyngeal compliance and, in one patient with OSA, during sleep.

Approvals for all measurements in human volunteers were obtained from The Human Research Ethics Committees of Sir Charles Gairdner Hospital and the University of Western Australia. Informed consent was obtained from all subjects.

\subsubsection{Description of anatomical optical coherence tomography (aOCT) In} this technique[12] an optical probe is placed inside a sealed, transparent catheter (3.0 $\mathrm{mm}$ outer diameter) that is inserted via the nares to the level of the midesophagus. In awake subjects the minor discomfort associated with the insertion of the catheter is minimized by application of topical anesthetic (10\% lidocaine spray) to the mucosa of the upper airway (throat and nose). Once in position, the catheter is taped to the external nares. The probe can be systematically moved within the catheter without displacing it and therefore without stimulating the airway mucosa. A minimum of 20 minutes is allowed for the effects of the lidocaine to remit before measurements are obtained. 
The system operates by directing a light beam perpendicular to the catheter. The distance between the probe head and the air-tissue interface of the airway wall is determined from the reflected light using a low-coherence optical interferometer. The probe rotates at $1.25 \mathrm{~Hz}$ to capture quantitative cross-sectional images of the upper airway lumen, in much the same way that a radar system captures an image. A customized computer program controls a motorized translation stage that allows the probe head to be precisely rotated and precisely translated to various levels within the pharynx to record cross-sections of interest. These tomograms can be viewed graphically in real-time on a personal computer or reconstructed in the form of a video. Additional details regarding the technique can be found in the online data supplement.

\subsubsection{Validation studies}

In vitro bench studies The accuracy of the technique was assessed in vitro in two ways. Firstly, the aOCT system was used to measure the CSA of circular holes of known size in a template constructed from wood. An aOCT scan was obtained with the probe fixed inside each hole, and the resulting images were thresholded to distinguish the interior region, then pixels counted (Matlab, Mathworks, Natick, MA, USA) to determine the CSA. Secondly, a phantom target was imaged simultaneously with $a \mathrm{OCT}$ and CT (third-generation CT scanner, GE HiSpeed CTi, GE Healthcare, Chalfont St. Giles, United Kingdom, with voltage and tube current set to optimize CT image quality, $80 \mathrm{kV}, 100 \mathrm{~mA}$ ). The phantom consisted of a paraffin wax block into which multiple holes were cut of varying shape and size. Wax was used as a material because it is readily shaped and because for CT it has similar properties to human tissue[30]. aOCT and CT cross-sectional images were obtained at the same location and orientation for each hole.

In vivo validation studies The system was then assessed in vivo. All in vivo studies were commenced with a 'pullback' scan. This was achieved by systematically retracting the optical probe at a constant speed $(0.2 \mathrm{~mm} / \mathrm{s})$ from the upper esophagus to the nasal cavity while the subject was breathing quietly. Each resulting data set was used to construct a movie of the pullback scan, thereby allowing convenient mapping of pharyngeal dimensions and anatomical landmarks, including specific sites for further investigation. For the studies described below, the optical probe was then moved to the relevant level in the airway to obtain detailed cross-sectional information for analysis.

aOCT vs CT Simultaneous $a \mathrm{OCT}$ and CT images of the upper airway were obtained in five healthy subjects without OSA ( 1 male and 4 females; mean age, 
27; BMI, 22.3). Subjects were awake, supine, breathing quietly with the head maintained in a neutral position using a Shea headrest (Gyrus ENT, Memphis, TN, USA). At the end of a normal exhalation (i.e., at functional residual capacity) each subject momentarily held his/her breath while simultaneous scans were obtained. The CT scanner was adjusted to scan through the tip of the probe orthogonal to the airway so that the $a \mathrm{OCT}$ and CT scan planes were coincident. Scans were obtained at the level of (i) the hypopharynx, $20 \mathrm{~mm}$ caudad to the tip of the epiglottis; (ii) the oropharynx, just craniad to the tip of the epiglottis; and (iii) the velopharynx (retropalatal pharynx), just craniad to the palatal rim.

Reproducibility, intra- and inter-observer variability The reproducibility of aOCT-derived measurements of pharyngeal dimensions was assessed by scanning five healthy subjects without OSA (1 male and 4 females; mean age, 27; BMI, 22.3) on two separate occasions (separated by $>24$ hours). On each occasion dimensions were calculated from $a \mathrm{OCT}$ images obtained at the same level at each of three identical locations (velo-, oro- and hypopharynx) within the upper airway.

To assess intra- and inter-observer variability of $a$ OCT-derived measurements of pharyngeal dimensions, 10 images of each of the velo-, oro- and hypopharynx were randomly selected from a database containing images from 10 subjects and analysed by two observers on two separate occasions (separated by $>24$ hours).

\subsubsection{Feasibility studies}

Measurement of pharyngeal compliance The feasibility of measuring pharyngeal compliance during wakefulness using the $a \mathrm{OCT}$ technique was investigated in ten subjects with OSA and eight non-snoring control subjects (see Table E1 for subject characteristics). Each individual was fitted with a nasal mask via which variable levels of continuous positive airway pressure (CPAP) could be applied. aOCT scans were performed and CSA at the velo-, oro- and hypopharynx measured while CPAP was maintained for two minutes at each of 3, 6, 9, 12 and 15 $\mathrm{cmH} 2 \mathrm{O}$. At each scanning level, CSA was plotted against applied mask pressure. The slope of this relationship (regression analyses) was defined as pharyngeal compliance. Separate area-pressure relationships were plotted for the minimum and maximum airway dimensions at each level.

aOCT scanning during sleep The feasibility of monitoring upper airway shape and size changes during sleep using $a \mathrm{OCT}$ was investigated in one male subject with severe OSA (AHI, 51; age 69; BMI, 25.9). This individual was instrumented for full overnight polysomnography[13] together with aOCT scanning. 
5.4.4 Analyses Analyses of $a$ OCT images were performed using ImageJ software (National Institutes of Health, Bethesda, Maryland, USA). The mucosallumen interface was manually traced and airway CSA calculated. Anteroposterior diameter was calculated in the mid-sagittal plane and lateral diameter was measured at the widest point in the coronal plane, perpendicular to the anteroposterior diameter.

Regression analyses were performed with the least-squares method. Measurements of pharyngeal compliance were compared between groups at each site using 2-way repeated measures ANOVA. A Holm-Sidak test was applied for all post-hoc comparisons. The Bland-Altman test [14] and the intra-class correlation coefficient [15] were used to evaluate the agreement between $a$ OCT and CTderived measurements of CSA from the wax phantom and pharyngeal dimensions, and to evaluate reproducibility, and intra- and inter-observer variability of aOCT-derived measurements.

\subsection{Results}

\subsubsection{Validation studies}

In vitro bench studies The percentage errors in $a \mathrm{OCT}$-derived measurements of CSA for five holes drilled in a template constructed from wood with radii of 5, 10, 15,20 and 25 millimetres were $0.3 \%, 0.6 \%, 0.3 \%,-2.7 \%$, and $-1.1 \%$, respectively. The mean error was $-0.5 \%$, or $0.1 \mathrm{~cm}^{2}$, which corresponded to a mean error in radius of $0.07 \mathrm{~mm}$.

The differences in CSA of 23 conduits of varying shape and size cut in a wax phantom are demonstrated in a Bland-Altman plot (Figure 5.1). The average CSA obtained using $a \mathrm{OCT}$ and CT was $610 \pm 500$ and $608 \pm 499 \mathrm{~mm}^{2}$, respectively. The mean difference between the measurements of CSA obtained with the two techniques was $2.1 \mathrm{~mm}^{2}$ with limits of agreement (2SD) from -13.2 to $17.4 \mathrm{~mm}^{2}$ and an intra-class correlation coefficient of 0.99 ( $p<0.001$ ). The mean difference between measurements of anteroposterior and lateral diameters were small, being 0.31 and $0.54 \mathrm{~mm}$, respectively. The intra-class correlation coefficient was 0.99 for measurements of anteroposterior $(\mathrm{p}<0.001)$ and lateral diameters $(\mathrm{p}<0.001)$.

In vivo validation studies Pullback scans Figure 5.2 shows lateral and anteroposterior longitudinal reconstructions from a pullback scan with six cross-sectional images at locations spaced at intervals along the pharynx. A movie created from the pullback scan (Video E1) is available in the online data supplement. A number of anatomical features are indicated on the images. The dark lines in the images 


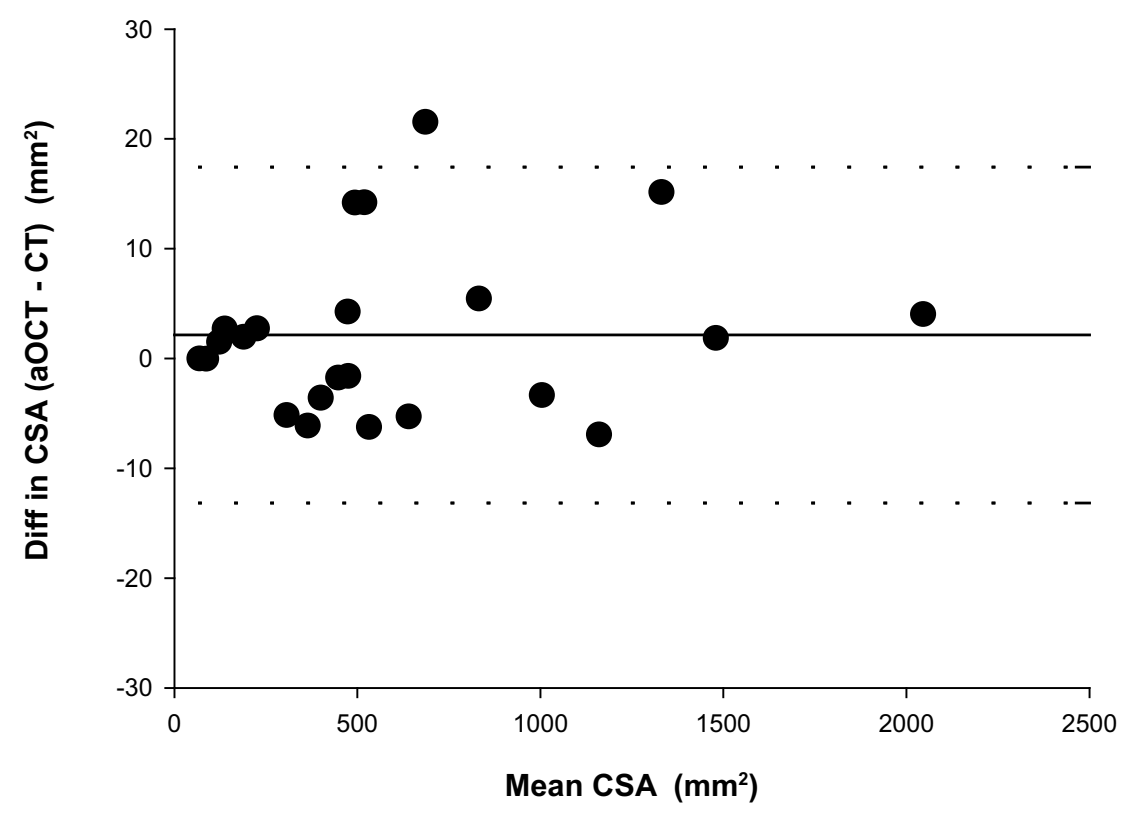

Figure 5.1: Bland-Altman plot of the agreement between measurements of cross-sectional area (CSA) derived from $a$ OCT and CT scans in 23 conduits of varying size and shape cut in a wax phantom. The solid line represents the mean difference between the two methods (systematic bias), and the dashed lines define the limits of agreement (2SD).

are the air-tissue boundaries of the lumen; for the cross-sections these are traced out by the $a \mathrm{OCT}$ system during a single rotation. The tissue-air boundaries in the figure appear fuzzy, because $a \mathrm{OCT}$ also detects subsurface reflections, but the air-airway wall interface is sharp, allowing accurate surface location.

$a$ OCT vs CT Figure 5.3 shows representative examples of simultaneous $a \mathrm{OCT}$ and CT scans at each of the three levels in one healthy subject without OSA. A Bland-Altman plot of the pooled difference in CSA using the two techniques in 5 healthy subjects is shown in Figure 5.4. The average CSA obtained using aOCT and CT was $133.2 \pm 61.4$ and $147.4 \pm 59.7 \mathrm{~mm}^{2}$, respectively. The mean difference between measurements obtained with the two techniques was $14.1 \mathrm{~mm}^{2}$ with limits of agreement (2SD) from -43.7 to $57.8 \mathrm{~mm}^{2}$. The mean difference in measures of anteroposterior and lateral diameters was 0.7 and $0.4 \mathrm{~mm}$, respectively, with limits of agreement from -3.5 to $4.8 \mathrm{~mm}$ and -6.8 to $7.6 \mathrm{~mm}$, respectively. The intra-class correlation coefficients for measurements of CSA and anteroposterior and lateral diameters were $0.89,0.66$ and 0.82 , respectively ( $\mathrm{p}<0.05$ for all). Data for measurements obtained at each of the three sites are shown in Table 1 and Tables E2 and E3 (online supplement). 


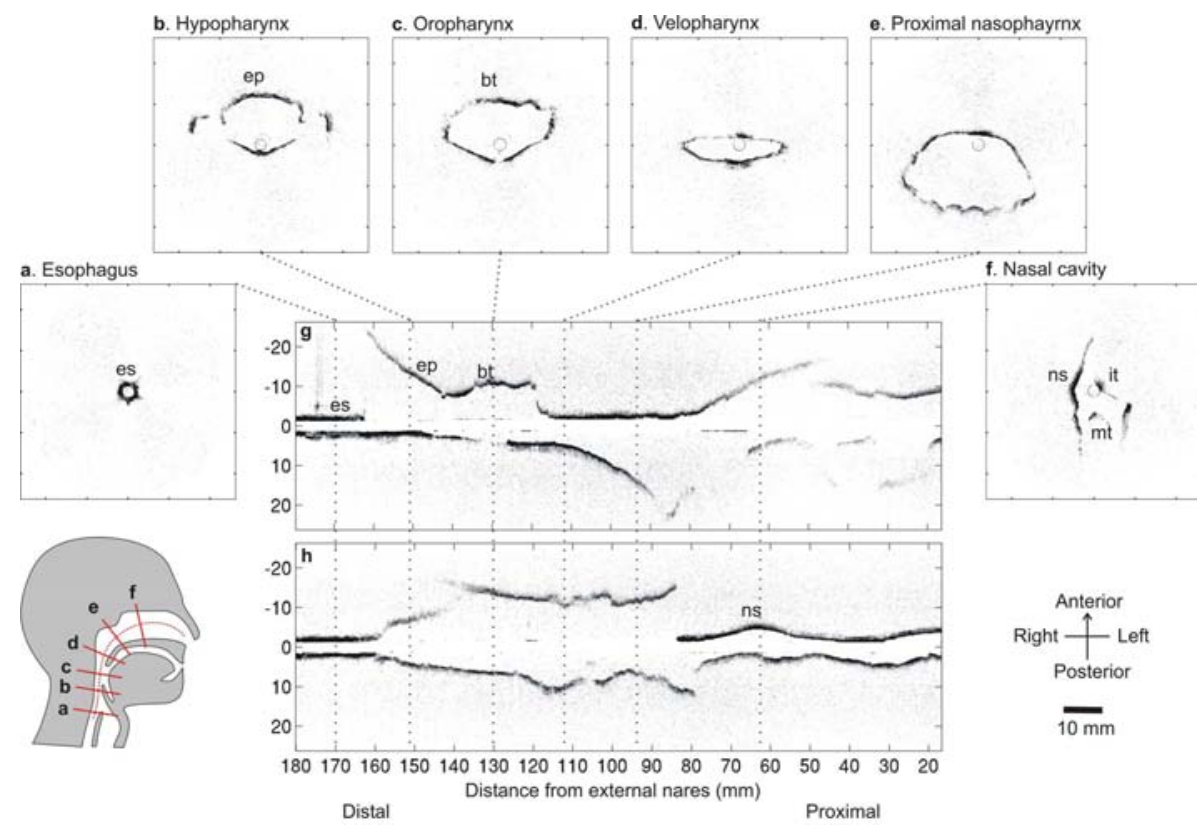

Figure 5.2: Selected images from a 'pullback scan' of a subject without sleep apnea. The 163-mm scan started in the upper esophagus $(180 \mathrm{~mm}$ from the external nares). (a-f) $52 \times 52 \mathrm{~mm}$ cross-sections selected to show various anatomical features of the pharynx and nasal cavity. $(\mathrm{g}, \mathrm{h})$ anteroposterior $(\mathrm{g})$ and lateral $(\mathrm{h})$ sections relative to the catheter. es, esophagus; ep, epiglottis; bt, base of tongue; ns, nasal septum; $\mathrm{mt}$, middle turbinate; and it, inferior turbinate.
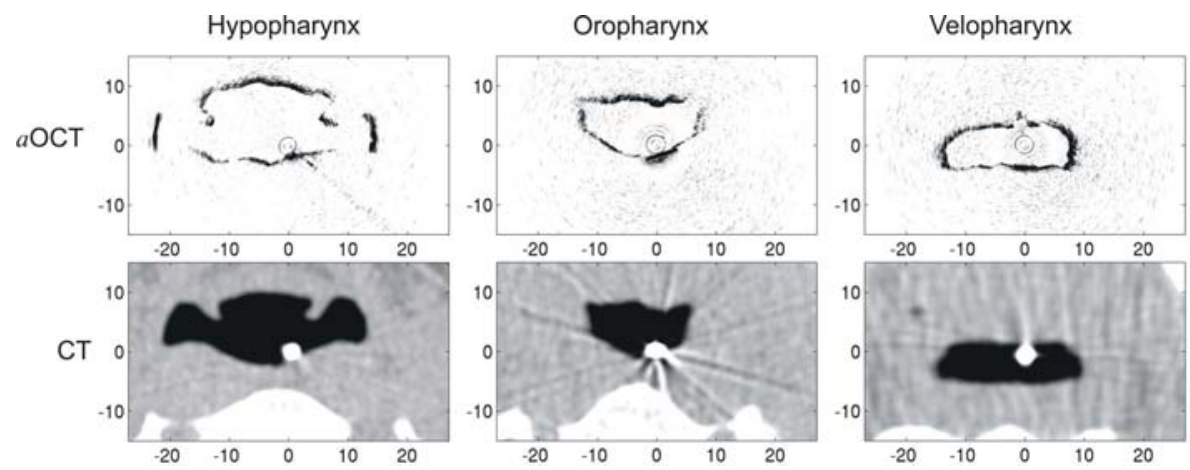

Figure 5.3: Simultaneous $a$ OCT scans and CT scans obtained in the hypopharynx, oropharynx and velopharynx. All images are shown on the same scale, with numbers indicating distance in millimeters.

Reproducibility To assess reproducibility, five individuals were scanned with aOCT on two separate occasions, and measurements of pharyngeal dimensions obtained at the same locations within the velo-, oro- and hypopharynx, using the same observer to derive the measurements. The average CSA on the first testing occasion was $161.2 \pm 43.6,226.9 \pm 42.8$, and $210.3 \pm 91.4 \mathrm{~mm}^{2}$, respectively, and 


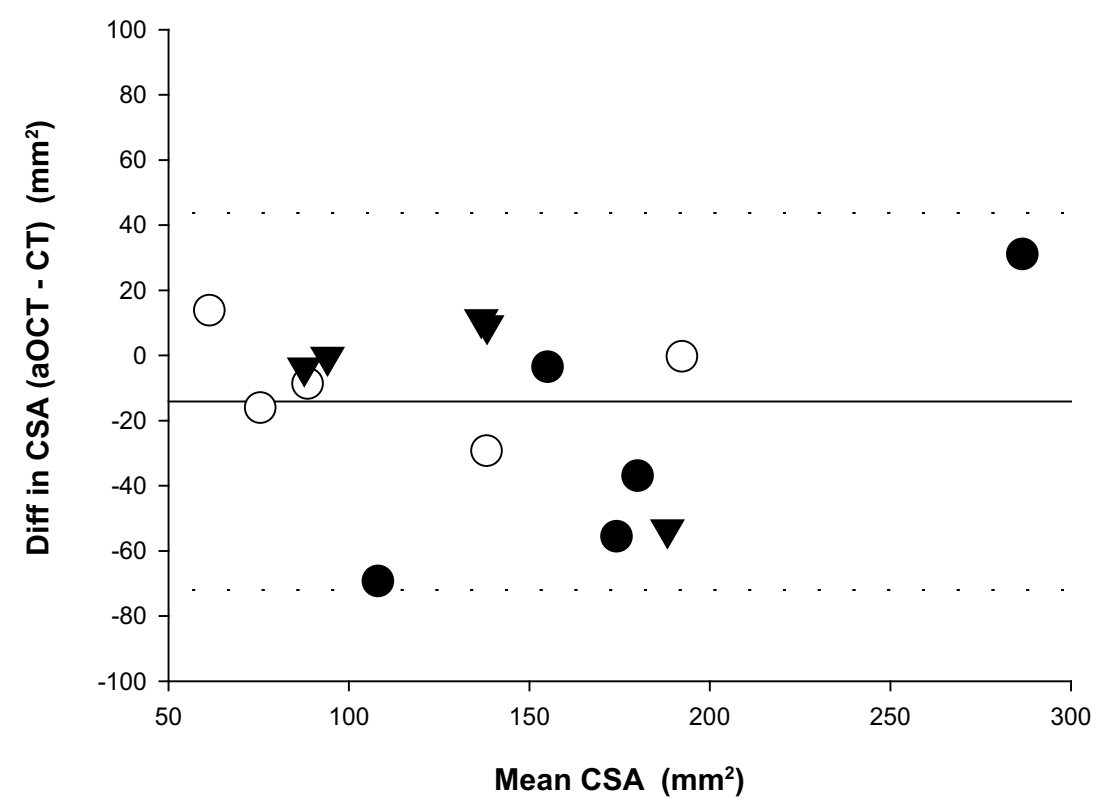

Figure 5.4: Bland-Altman plots of the agreement between measurements of cross-sectional area (CSA) derived from simultaneous aOCT and CT scans in the hypopharynx (closed circles), oropharynx (open circles) and velopharynx (closed triangles) in 5 healthy subjects without sleep apnea. The solid line represents the mean difference between the two methods (systematic bias), and the dashed lines define the limits of agreement $( \pm 2 \mathrm{SD})$.

$167.9 \pm 60.8,242.6 \pm 45.7$, and $188.1 \pm 93.3 \mathrm{~mm}^{2}$ on the second occasion. The mean difference between the two testing days was $6.7,15.7$, and $22.2 \mathrm{~mm}^{2}$, respectively, and the intra-class correlation coefficients for measurements obtained on the two testing days were $0.75,0.70$ and 0.97 , respectively ( $\mathrm{p}<0.01$ for all) (Table 1 ). The mean difference and intra-class correlation coefficients between duplicate measurements of anteroposterior and lateral diameters are reported in Tables E2 and E3 (online supplement).

Intra-observer variability To assess intra-observer variability, a total of 10 $a \mathrm{OCT}$ images were randomly selected for measurement of pharyngeal dimensions at the level of the velo-, oro- and hypopharynx on two separate occasions by each of two observers. The average CSA on the first measurement occasion was $180.3 \pm 121.5,284.1 \pm 167.8$, and $452.4 \pm 135.3 \mathrm{~mm}^{2}$, respectively, and $178.8 \pm 120.5$, $281.4 \pm 167.4$, and $451.8 \pm 131.7 \mathrm{~mm}^{2}$ on the second occasion. The mean difference (pooled data) between these duplicate measurements was small, being 1.4, 2.7, and $0.6 \mathrm{~mm}^{2}$, respectively, and the intra-class correlation coefficients were 0.99 
for all ( $\mathrm{p}<0.001$ for all) (Table 1). The mean differences and intra-class correlation coefficients between duplicate measurements of anteroposterior and lateral diameters were also small (see online supplement, Table E2 and E3).

Inter-observer variability To assess inter-observer variability, the results of the analyses of the images by the two observers were compared. The average CSA at the velo-, oro- and hypopharynx was $180.9 \pm 120.9,281.4 \pm 166.2$, and $450.2 \pm 131.4 \mathrm{~mm}^{2}$, respectively, from observer one, and 179.2 $\pm 121.2,284.0 \pm 169.0$, and $454.0 \pm 135.6 \mathrm{~mm}^{2}$ from observer two. The mean difference between the two observers was $0.7,2.6$, and $3.9 \mathrm{~mm}^{2}$, respectively, and the intra-class correlation coefficients were 0.99 for all ( $\mathrm{p}<0.001$ for all) (Table 1). The mean differences between the observers for measurements of anteroposterior and lateral diameters were also small (see online supplement, Table E2 and E3).

\subsubsection{Feasibility studies}

Measurement of pharyngeal compliance The slope of the relationship describing change in upper airway size with increasing airway pressure defined pharyngeal compliance (Figure 5.5). CSA varied with phase of respiration. Using maximal CSA, measures of compliance at the velo-, oro- and hypopharynx were $5.8 \pm 1.9,7.1 \pm 1.8$ and $9.7 \pm 1.9 \mathrm{~mm}^{2} / \mathrm{cmH} 2 \mathrm{O}$ for controls and $2.3 \pm 1.6,7.5 \pm 1.6$ and $12.3 \pm 1.6 \mathrm{~mm}^{2} / \mathrm{cmH} 2 \mathrm{O}$ for individuals with OSA ( $\mathrm{p}<0.05$ for velo- vs hypopharynx, oro- vs hypopharynx and velo- vs oropharynx in the OSA group only). Using minimal CSA, measures of compliance at the velo-, oro- and hypopharynx were $4.1 \pm 2.0,5.8 \pm 1.8$ and $8.1 \pm 2.0 \mathrm{~mm}^{2} / \mathrm{cmH} 2 \mathrm{O}$ for controls and $2.2 \pm 1.6$, $6.4 \pm 1.6$ and $12.1 \pm 1.6 \mathrm{~mm}^{2} / \mathrm{cmH} 2 \mathrm{O}$ for individuals with OSA ( $\mathrm{p}<0.05$, velo- vs hypopharynx and oro- vs hypopharynx in the OSA group only). A movie (Video E2) of a representative pharyngeal compliance study for one subject is available in the online data supplement.

aOCT scanning during sleep Figure 5.6 demonstrates changes in upper airway size and shape before, during and after airway collapse (i.e. an apneic event) in an individual with OSA. Images were collected in the velopharynx while the subject was in stable Stage 2 sleep. The ten selected aOCT cross-sectional images in Figure 5.6 (a-j) show: the airway changing size during normal unobstructed breathing, being smaller during inspiration than expiration (a-d); the airway narrowing (e) then collapsing during a 5-second apnea (f); collapsing during a 12second apnea $(g, h)$; and restoration of an open airway following momentary arousal from sleep ( $i, j$ ). A movie (Video E3) showing aOCT data of the apneic event is available in the online data supplement. 


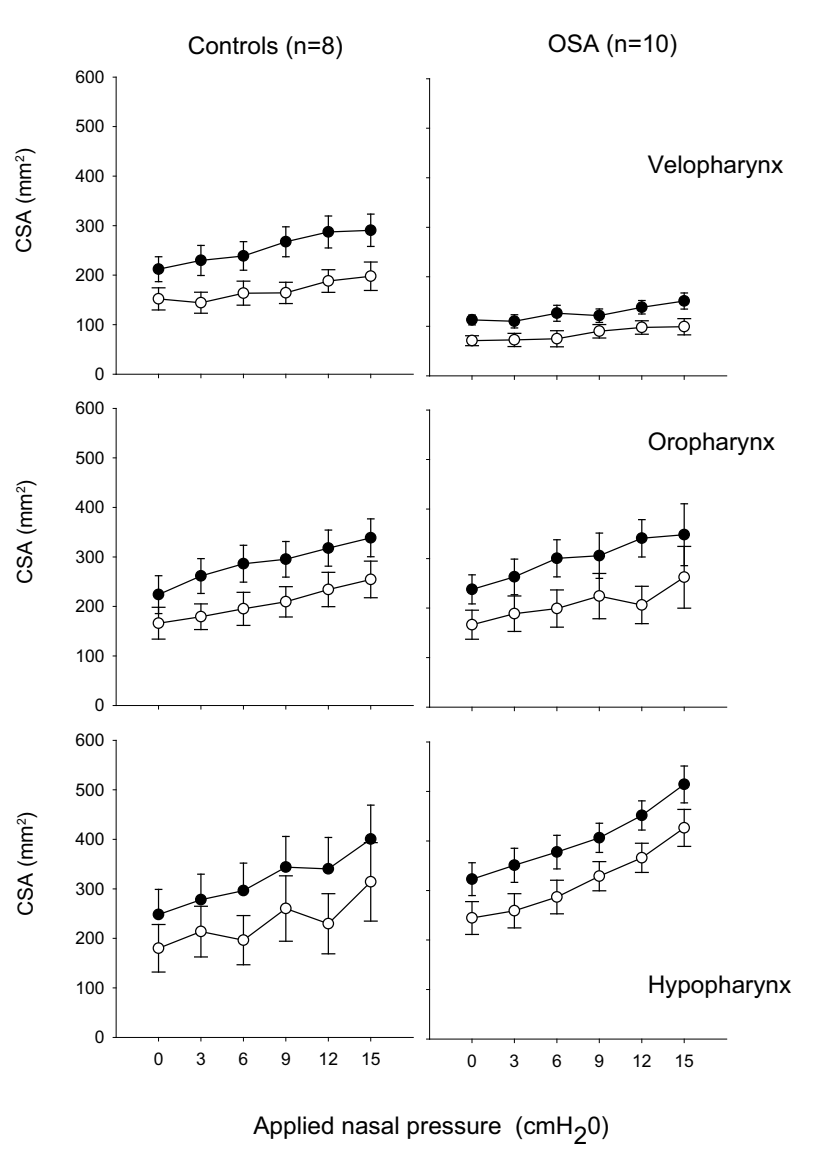

Figure 5.5: The effect of changing airway pressure on maximum (closed circles) and minimum (open circles) cross-sectional area (CSA) at the level of the velo- oro- and hypopharynx. Measurements were obtained during wakefulness in subjects without ( 5 female, 3 male) and with sleep apnea ( 4 female, 6 male); mean data \pm SE. Subjects were not matched for age, sex or body mass index, thus comparison of CSA between groups is not meaningful.

\subsection{Discussion}

This study reports on a new optical technique that can provide quantitative real-time imaging of the internal anatomy of the human upper airway. The impetus for this work has been the long-recognized need to quantitatively evaluate changes in human upper airway size and shape during sleep. Such measurements are of particular value in understanding the pathophysiology of OSA and in devising, applying and determining the effectiveness of treatment modalities1.

In recent years, upper airway size and shape have been measured by a number of techniques, including CT [16-18], fluoroscopy [19,20], MRI [21-24], nasoendoscopy [25-28], and acoustic reflection [4,29,30]. While these studies have demonstrated important differences in pharyngeal shape and size between indi- 


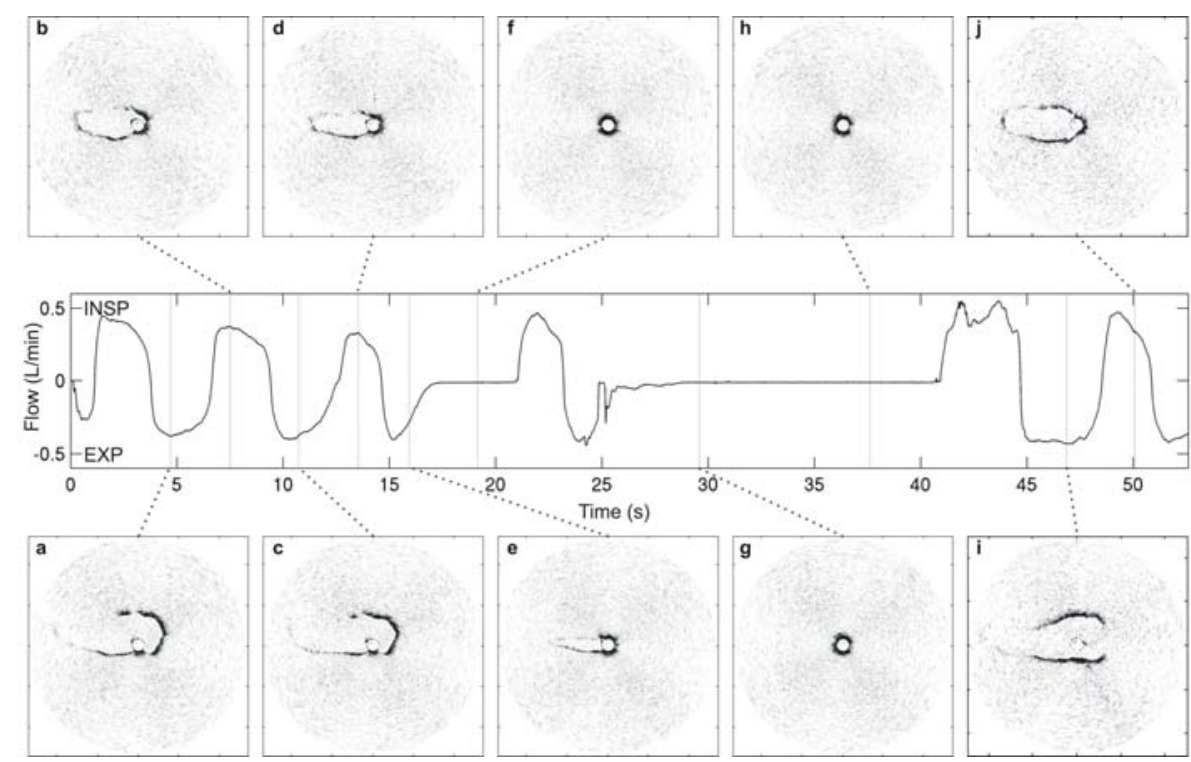

Figure 5.6: Changes in upper airway caliber before, during and after airway collapse (i.e. an apneic event) in an individual with obstructive sleep apnea. The 5- and 12-second apneic events are clearly observed in both the respired air flow recording (centre plot) and the series of airway images (a-j). INSP and EXP refer, respectively, to inspiration and expiration.

viduals with and without OSA, in terms of baseline anatomy $[4,16,18,22,23,29,30]$ and dynamic behavior $[17,19-21,24-28]$, the routine use of these technologies for study of the upper airway on repeated occasions and over prolonged periods in individuals is limited. We present $a \mathrm{OCT}$ as a useful addition to these existing technologies. Its minimal invasiveness, quietness, comfort and lack of potentially hazardous effects (such as ionizing radiation) make it suitable for study of pharyngeal size and shape over prolonged periods during both sleep and wakefulness.

The in vitro measurements illustrate the precision of the method in obtaining measurements of shape and size. The differences between $a \mathrm{OCT}$ and CT derived measures of anteroposterior and lateral dimensions were very small $(0.4 \mathrm{~mm}$ on average) and the high intra-class correlation coefficients demonstrate that the two techniques provide comparable measurements. CSA measurements tended to be slightly larger when measured using aOCT (by $2.1 \mathrm{~mm}^{2}$ on average), although the high intra-class correlation coefficients demonstrate that the two techniques show good agreement.

In each of the in vivo studies presented in this paper, aOCT scanning was commenced with a pullback scan (Fig. 2). This was achieved by systematically 
retracting the optical probe from the upper esophagus to the nasal cavity while the subject was breathing quietly. There is no sensation of movement of the probe because it is retracted within a fixed catheter, thereby avoiding stimulation of the airway mucosa. Also, because the mass of the rotating fibre probe is exceedingly low, and substantially less than the plastic catheter, significant mechanical vibration does not transfer from the probe to the catheter. The benefit of this was demonstrated in the one subject who was studied during sleep (see Figure 5.6), where the probe was moved to various sites in the upper airway without causing arousal.

On completion of the pullback scan, each resulting data set was then used to construct a movie (see Video E1). While the movie constitutes a useful data set in its own right, it can also be used to identify specific sites for further investigation. In such circumstances the optical probe is moved to those sites and continuous scanning undertaken to monitor the upper airway during steady-state conditions, state changes (e.g., changes in sleep stage or posture), or imposed changes (e.g., changes in airway pressure).

aOCT generated images of pharyngeal cross-sections that were comparable to images obtained by radiographic CT. The differences between $a$ OCT and CT derived measures of anteroposterior and lateral dimensions were small $(0.8 \mathrm{~mm}$ on average) at all levels. CSA measurements tended to be smaller with $a \mathrm{OCT}$, particularly at the level of the hypopharynx. The most likely contributors to this difference were the misalignment between the $a \mathrm{OCT}$ and CT scan planes (caused by restricted movement of the CT gantry and/or occasional head/neck movement between the time of the CT scout scan and the cross-sectional scan), and obscuration of small parts of the airway wall during aOCT scanning (particularly at the hypopharynx, Fig. 3). This obscuration, which is a limitation of the aOCT technique (see below), may also have contributed to the larger difference in CSA of the hypopharynx relative to other levels when individuals were measured on two separate occasions. Other contributors to this variability include position of the tongue and soft palate, and differences in head and neck position, although the latter were minimized as far as possible between the two testing occasions.

5.6.1 Methodological considerations Measurement of upper airway anatomy using a OCT requires insertion of a catheter of similar dimensions to a nasogastric tube, within which the probe is housed. It is possible that the presence of the catheter may alter airway behavior, disturb sleep and alter sleep architecture. While our study did not specifically address this issue, previous investi- 
gations have shown that overnight catheter-based measurements of esophageal pressure, the current reference standard for measuring respiratory effort [31] (i) is acceptable in the majority of patients $(96 \%)$ with catheter discomfort and insertion difficulty being extremely uncommon [32], (ii) has no significant effect on sleep quality or on respiratory events [33] and, (iii) has minimal effect on sleep architecture [34]. The size of the catheter in those studies was approximately 1.9 $\mathrm{mm}$ in diameter. It is probable that the larger the catheter the greater the potential for sleep disturbance. This was likely to be the case in the study by Hessel et al. [35] who, when using a catheter of $4.0 \mathrm{~mm}$ diameter, reported that only half of their patients completed a whole night with the tube in place. The diameter of the current $a \mathrm{OCT}$ catheter is $3.0 \mathrm{~mm}$. The optics system we currently use has an outer diameter of $1.3 \mathrm{~mm}$. It is feasible to house the system in a narrower plastic catheter of $1.5 \mathrm{~mm}$ internal diameter and $2.0 \mathrm{~mm}$ outer diameter.

aOCT has the capacity to continuously measure changes in airway dimensions under a variety of conditions. Such changes can be seen during tidal breathing in each of the supplementary videos, and before, during and after an apneic event in an individual with OSA (Figure 5.6). The images obtained from this individual show the potential of the technique to describe the patterns of change in airway shape and size which lead up to upper airway collapse. In this individual, the upper airway narrowed and then closed during expiration (e.g. frames e and f), consistent with previous analyses of airflow data [36], nasoendoscopy images [37], and CT images [38].

When CSA is related to changes in airway pressure, a measure of pharyngeal compliance can be derived as shown in Figure 5.5. The finding that, in individuals with OSA, the pharynx was smallest and least compliant at the level of the velopharynx is consistent with a previous CT-based study [39]. While the 1.25 $\mathrm{Hz}$ rotation rate of the $a \mathrm{OCT}$ system is comparable with the acquisition rates of most CT and MRI scanners it is not sufficient to track changes in airway caliber when breathing rates are rapid. This can be allowed for by synchronizing the aOCT images with other respiratory data, such as flow or volume change, and multiplexing images obtained over several breaths, so that the composite image is obtained relative to a particular phase of respiration. The need to do this would be obviated by increasing the rotation rate by at least a factor of three, which is an intended technological development.

A limitation of the technique is its capacity to view the complete circumference of the airway at all sites in all individuals. In some individuals, on some 
occasions, the probe may be positioned adjacent to an airway wall that is sufficiently concave or irregular to obscure parts of the airway wall from the probe light beam. An example of this is the hypopharyngeal region (see Figs. 2, 3), in which the epiglottis can shadow the lower part of the retroglossal pharyngeal wall. In our experience, partial airway profiles occur most commonly at this level, but rarely in the velopharynx, where such gross irregularity of the airway is uncommon (see Figs. 2, 3). Where obscuration does limit measurement of parameters such as total pharyngeal area at that specific site, other useful parameters such as lateral extent of the airway are usually available.

Depending on the particular airway and location of the catheter, the scan range required to fully capture the airway cross-section could exceed a $26 \mathrm{~mm}$ radius (i.e. $52 \mathrm{~mm}$ total cross-section width), the current limit for our system. This is not a major limitation as individuals with OSA typically have either smaller pharynxes overall or a segment of the pharynx which is narrower than average $[16,23]$. Nevertheless, it is possible to modify the instrument to extend the range in the future.

A challenge to imaging dimensions of the airway using aOCT is that there is longitudinal motion of the airway walls. It is possible, therefore that the actual level of the structures that a fixed probe is measuring may vary over time as the airway structure moves in a ventro-caudal direction. Such movement was recently demonstrated by Liao et al., [40] who used cephalometry to document pharyngeal motion during a Mueller maneuver in a group of patients with sleep-disordered breathing. They showed that, relative to measurements made at end-expiration, a Mueller maneuver increased the length of the pharyngeal airway by $2 \%$ in patients with mild OSA and by $6 \%$ in patients with moderate-tosevere OSA. The longitudinal and transmural forces generated during a wakeful Mueller maneuver most likely represent an extreme load on the upper airway, out of proportion to those produced by tidal breathing or by an obstructive event during sleep. Nevertheless, the effect of such longitudinal motion of the airway walls on $a$ OCT-derived measurements of pharyngeal shape and size will need to be considered, perhaps by relating measurements to anatomical landmarks (such as the epiglottis tip).

5.6.2 Future applications Applied to clinical and research investigations of OSA, $a$ OCT may allow determination of sites of and anatomical predispositions to airway collapse, the length of the collapsing regions and the relationship of collapse to transmural pressure gradients, phase of respiration and state of sleep, 
without restrictions on time or repetition. The capacity of $a$ OCT to measure pharyngeal size and shape dynamically and repeatedly over extended periods at the bedside during sleep and wakefulness may provide useful additional information to aid OSA diagnosis, and allow treatment to be devised on the basis of individual airway characteristics.

\subsection{Acknowledgements}

We thank the following individuals for their support and contributions to this work: Kathleen Maddison and Kelly Shepherd from the Department of Pulmonary Physiology, Sir Charles Gairdner Hospital; Peter Muir, Neil Hicks and Associate Professor Vincent Low from the Department of Radiology, Sir Charles Gairdner Hospital; Alexandre Paduch, Stefan Schwer and Jonathan Ng from the School of Electrical, Electronic and Computer Engineering, University of Western Australia.

\subsection{References}

1. Leiter J. Upper airway shape: Is it important in the pathogenesis of obstructive sleep apnea? Am J Respir Crit Care Med 1996;153:894-8.

2. Rama AN, Tekwani SH, Kushida CA. Sites of Obstruction in Obstructive Sleep Apnea. Chest 2002;122:1139-47.

3. Badr MS, Toiber F, Skatrud JB, Dempsey J. Pharyngeal Narrowing Occlusion During Central Sleep-Apnea. J Appl Physiol 1995;78:1806-15.

4. Hoffstein V, Fredberg JJ. The acoustic reflection technique for noninvasive assessment of upper airway area. Eur Respir J 1991;4:602-11.

5. Huang D, Swanson EA, Lin CP, Schuman JS, Stinson WG, Chang W, Hee MR, Flotte T, Gregory K, et al. Optical Coherence Tomography. Science 1991;254:117881.

6. Drexler W, Morgner U, Ghanta RK, Krtner FX, Schuman JS, Fujimoto JG. Ultrahigh-resolution ophthalmic optical coherence tomography. Nat Med 2001;7:5027.

7. Bechara FG, Gambichler T, Stucker M, Orlikov A, Rotterdam S, Altmeyer P, Hoffmann K. Histomorphologic correlation with routine histology and optical coherence tomography. Skin Res Technol 2004;10:169-73.

8. Fujimoto JG, Boppart SA, Tearney GJ, Bouma BE, Pitris C, Brezinski ME. High resolution in vivo intra-arterial imaging with optical coherence tomography. Heart 1999;82:128-33. 
9. Pitris C, Jesser C, Boppart SA, Stamper D, Brezinski ME, Fujimoto JG. Feasibility of optical coherence tomography for high-resolution imaging of human gastrointestinal tract malignancies. J Gastroenterol 2000;35:87-92.

10. Pan Y, Lavelle JP, Bastacky SI, Meyers S, Pirtskhalaishvili G, Zeidel ML, Farkas DL. Detection of tumorigenesis in rat bladders with optical coherence tomography. Med Phys 2001;28:2432-40.

11. Bouma BE, Tearney GJ. Handbook of optical coherence tomography. New York: Marcel Dekker; 2002.

12. Armstrong JJ, Leigh MS, Walton ID, Zvyagin AV, Alexandrov SA, Schwer S, Sampson DD, Hillman DR, Eastwood PR. In vivo size and shape measurement of the human upper airway using endoscopic long-range optical coherence tomography. Opt Expr 2003;11:1817-26.

13. Polysomnography Task Force of the American Sleep Disorders Association Standards of Practice Committee. Practice parameters for the indications for polysomnography and related procedures. Sleep 1997;20:406-22.

14. Bland JM, Altman DG. Statistical methods for assessing agreement between two methods of clinical measurement.[see comment]. Lancet 1986;1:307-10.

15. Bland JM, Altman DG. Measurement error and correlation coefficients. BMJ 1996;313:41-2.

16. Haponik EF, Smith PL, Bohlman ME, Allen RP, Goldman SM, Bleecker ER. Computerized tomography in obstructive sleep apnea. Correlation of airway size with physiology during sleep and wakefulness. Am Rev Respir Dis 1983;127:2216.

17. Shepard JW, Jr., Stanson AW, Sheedy PF, Westbrook PR. Fast-CT evaluation of the upper airway during wakefulness in patients with obstructive sleep apnea. Prog Clin Biol Res 1990;345:273-82.

18. Lowe AA, Fleetham JA, Adachi S, Ryan CF. Cephalometric and computed tomographic predictors of obstructive sleep apnea severity.[see comment]. Am J Orthod Dentofacial Orthop 1995;107:589-95.

19. Tsushima Y, Antila J, Svedstrom E, Vetrio A, Laurikainen E, Polo O, Kormano M. Upper airway size and collapsibility in snorers: evaluation with digital fluoroscopy. Eur Respir J 1996;9:1611-8.

20. Wheatley JR, Kelly WT, Tully A, Engel LA. Pressure-diameter relationships of the upper airway in awake supine subjects. J Appl Physiol 1991;70:2242-51.

21. Arens R, Sin S, McDonough JM, Palmer JM, Dominguez T, Meyer H, Wootton DM, Pack AI. Changes in upper airway size during tidal breathing in children 
with obstructive sleep apnea syndrome. Am J Respir Crit Care Med 2005;171:1298304.

22. Schwab RJ, Pasirstein M, Pierson R, Mackley A, Hachadoorian R, Arens R, Maislin G, Pack AI. Identification of Upper Airway Anatomic Risk Factors for Obstructive Sleep Apnea with Volumetric Magnetic Resonance Imaging. Am J Respir Crit Care Med 2003;168:522-30.

23. Schwab RJ, Gupta KB, Gefter WB, Metzger LJ, Hoffman EA, Pack AI. Upper airway and soft tissue anatomy in normal subjects and patients with sleepdisordered breathing. Significance of the lateral pharyngeal walls. Am J Respir Crit Care Med 1995;152:1673-89.

24. Donnelly LF, Surdulescu V, Chini BA, Casper KA, Poe SA, Amin RS. Upper Airway Motion Depicted at Cine MR Imaging Performed during Sleep: Comparison between Young Patients with and Those without Obstructive Sleep Apnea. Radiology 2003;227:239-45.

25. Isono S, Morrison DL, Launois SH, Feroah TR, Whitelaw WA, Remmers JE. Static mechanics of the velopharynx of patients with obstructive sleep apnea. J Appl Physiol 1993;75:148-54.

26. Rowley JA, Sanders CS, Zahn BR, Badr MS. Effect of REM sleep on retroglossal cross-sectional area and compliance in normal subjects. J Appl Physiol 2001;91:239-48.

27. Guilleminault C, Hill MH, Simmons FB, Powell N, Riley R, Stoohs R. Passive constriction of the upper airway during central apneas: fiberoptic and EMG investigations. Respir Physiol 1997;108:11-22.

28. Morrell MJ, Badr MS. Effects of NREM sleep on dynamic within-breath changes in upper airway patency in humans. J Appl Physiol 1998;84:190-9.

29. Mohsenin V. Gender differences in the expression of sleep-disordered breathing : role of upper airway dimensions.[see comment]. Chest 2001;120:1442-7.

30. Kamal I. Acoustic pharyngometry patterns of snoring and obstructive sleep apnea patients. Otolaryngol Head Neck Surg 2004;130:58-66.

31. Taskforce. Sleep-related breathing disorders in adults: recommendations for syndrome definition and measurement techniques in clinical research. The Report of an American Academy of Sleep Medicine Task Force. Sleep 1999;22:66789.

32. Oeverland B, Akre H, Kvaerner KJ, Skatvedt O. Patient discomfort in polysomnography with esophageal pressure measurements. Eur Arch Oto-Rhino-L 2005;262:2415. 
33. Skatvedt O, Akre H, Godtlibsen OB. Nocturnal polysomnography with and without continuous pharyngeal and esophageal pressure measurements. Sleep 1996;19:485-90.

34. Chervin RD, Aldrich MS. Effects of esophageal pressure monitoring on sleep architecture.[see comment]. Am J Respir Crit Care Med 1997;156:881-5.

35. Hessel NS, Laman M, van Ammers VC, van Duijn H, de Vries N. Feasibility study of Flextube reflectometry for localisation of upper airway obstruction in obstructive sleep apnea. Rhinology 2003;41:87-90.

36. Sanders MH, Rogers RM, Pennock BE. Prolonged expiratory phase in sleep apnea. A unifying hypothesis. Am Rev Respir Dis 1985;131:401-8.

37. Morrell MJ, Arabi Y, Zahn B, Badr MS. Progressive retropalatal narrowing preceding obstructive apnea. Am J Respir Crit Care Med 1998;158:1974-81.

38. Schwab RJ, Gefter WB, Hoffman EA, Gupta KB, Pack AI. Dynamic upper airway imaging during awake respiration in normal subjects and patients with sleep disordered breathing. Am Rev Respir Dis 1993;148:1385-400.

39. Kuna ST, Bedi DG, Ryckman C. Effect of nasal airway pressure on upper airway size and configuration. Am Rev Respir Dis 1988;138:969975.

40. Liao YF, Huang CS, Chuang ML. The utility of cephalometry with the Muller maneuver in evaluating the upper airway and its surrounding structures in Chinese patients with sleep-disordered breathing. Laryngoscope 2003;113:614-9.

\subsection{Online data supplement}

\subsubsection{Methods}

System description The distance ranging technique used in the $a \mathrm{OCT}$ system is an optical interferometric method that accurately measures the time of flight and intensity of light scattered from a biological sample. In the catheter-based configuration used here, it determines the distance from the probe head to the internal lumen surface [E1]. This is achieved with a fiber-optic Mach-Zehnder interferometer (see Figure E1) in which a light wave is split into two paths; a reference path that has a precise, controllable path length, and a sample path terminated by biological tissue (the hollow organ in this case). The recombination of the reference and scattered light waves at a photodetector yields an interference signal that corresponds to matched optical lengths (optical length is the product of physical length and refractive index) of the reference and sample paths, to within the light source coherence length [E2]. As the reference path length is scanned, the interference signals are analyzed to determine the locations of the reflections in 
the sample. The heterodyne-detection advantage of the interferometric technique has the advantage of both high distance-ranging resolution and sensitivity to very weak reflections [E2]. The bandwidth and centre wavelength of the light source used determines the achievable distance resolution. In the present system, the 21 $\mathrm{mW}$ superluminescent diode source has a Gaussian-shaped spectrum centered at $1301 \mathrm{~nm}$. The distance-ranging resolution is inversely related to the spectral width [E2,E3]. In this case the spectral width (full-width half-maximum) of 34.2 $\mathrm{nm}$, which gave a distance-ranging resolution (also full-width half-maximum) of $22 \mu \mathrm{m}$.

A long-range scanning frequency-domain delay line is used in the reference arm of the interferometer [E1,E4-6]. This delay line employs a galvanometer mirror driven by a triangular waveform at $250 \mathrm{~Hz}$, which results in an axial scan speed of $12.5 \mathrm{~m} / \mathrm{s}$. It is the scanning of the galvanometer mirror in the delay line which performs the distance ranging measurement 250 times per second. The delay line allows the system to range up to $27 \mathrm{~mm}$ in total ( $26 \mathrm{~mm}$ from the probe), giving cross-sectional images of up to $52 \mathrm{~mm}$ in diameter, which allows the capture of full airway profiles in almost all subjects of clinical interest. As long as the probe can detect the whole circumference of the airway wall, the location of the catheter in the airway does not affect the dimension measurements. The system accurately measures the physical geometry of the $360^{\circ}$ airway cross-section so the recorded lumen size and shape is independent of the probe position. The $a \mathrm{OCT}$ system detects optical scattering from the air-tissue interface and up to several hundred micrometers of the epithelial tissue. The heterodyne carrier is generated by modulation at $1.2 \mathrm{MHz}$ using an integrated optic phase modulator. The detected interference signal from balanced photodetectors is band pass filtered and logarithmically demodulated before digitization by a data acquisition card. The sensitivity of the optical ranging system was measured to be $-98 \mathrm{~dB}$, signifying that the system is able to detect a surface when only $0.6 \mathrm{pW}$ of optical power scattered from the airway wall is collected by the probe. It is important to be sensitive to weak power levels because, by necessity, the probe must be small while the distances are relatively large; therefore, its light-gathering power (numerical aperture) is low. The optical power launched from the probe is $4 \mathrm{~mW}$. This is well below the $125 \mathrm{~mW}$ maximum permissible exposure for tissue at this wavelength and scanning speed [E7].

The transverse-scanning catheter-mounted probe consists of single-mode optical fiber supported by a biplex stainless steel torque coil, terminated by a micro- 
optical assembly which collimates the light and deflects it $90^{\circ}$ to the catheter axis. The distal end micro-assembly consists of a 0.276-pitch GRIN lens of $1.0 \mathrm{~mm}$ diameter attached to the optical fiber, with a $0.7 \mathrm{~mm}$ right-angle prism attached to the lens to deflect the beam transverse to the probe. The probe assembly is located within a transparent polyvinylchloride catheter of $3.0 \mathrm{~mm}$ outer diameter. The beam spot size at its waist located at $8.4 \mathrm{~mm}$ from the probe is $0.18 \mathrm{~mm}$. The beam waist varies from $0.18 \mathrm{~mm}$ to $0.24 \mathrm{~mm}$ over the $27 \mathrm{~mm}$ range. The lateral resolution, limited by the beam size and the delay-line scan repetition rate, varies from 0.18 to $0.42 \mathrm{~mm}$ over this same range. The probe is connected to the motorized translation stage allowing it to be precisely rotated and translated to various levels within the pharynx to record cross-sections at sites of interest. A fiber-pigtailed optical rotary joint connects the probe optical fiber to the fiber interferometer. The torque coil supporting the optical fiber is designed to ensure that the rotation generated at the probes proximal end is faithfully transmitted to its distal end. The rotation rate of the probe is $1.25 \mathrm{~Hz}$. To capture a complete three-dimensional measurement of the entire upper airway (data not shown) the probe is pulled back along the catheter at a constant speed $(0.2 \mathrm{~mm} / \mathrm{s})$ while capturing both optical ranging data and probe position. The pullback speed is controlled by a synchronous microprocessor and precision stepper motor. During this process (a "pullback scan") the probe beam captures information in a helical pattern, similar to axial CT except that the axis follows the catheter.

A custom $\mathrm{C}++$ program acquires the data, generates a live rendering of the cross-sectional data and controls all probe movement. The images are captured at the rate of rotation of the probe. At each angle 5400 voxels are captured. The program is multi-threaded to cope with the demands of transferring and saving the large amount of data (162 megabytes per minute) as well as generating the real-time polar image reconstruction and responding to the user interface. Along with the image data, the software allows experimental conditions and patient information to be recorded. A time stamping function allowed the $a \mathrm{OCT}$ scans to be precisely synchronized with Powerlab (ADInstruments) recordings of physiological parameters, including respired air flow (pneumotachograph), lung volume change (respiratory inductance plethysmograph), electroencephalograms (EEG), electromyograms (EMG), and electrooculograms (EOG).

5.9.2 In vitro validation studies The baseline accuracy of the technique was assessed by experiments involving two custom-built phantoms.

Firstly, the aOCT system was used to measure the CSA of circular holes of 
known size in a template constructed from wood. Circular holes with radii of 5, 10, 15, 20 and 25 millimetres were drilled in a sheet of wood. An aOCT scan was obtained with the probe fixed inside each hole. The resulting images were thresholded to distinguish the interior region, then pixels counted (Matlab, Mathworks, Natick, MA, USA) to determine the CSA.

Secondly, a phantom target was imaged simultaneously with aOCT and CT (third-generation CT scanner, GE HiSpeed CTi, with voltage and tube current set to optimize CT image quality, $80 \mathrm{kV}, 100 \mathrm{~mA}$ ). The phantom consisted of a paraffin wax block into which multiple holes were cut of varying shape and size. Wax was used as a material because it is readily shaped and because for CT it has similar properties to human tissue. aOCT and CT cross-sectional images were obtained at the same location and orientation for each hole (Figure E2).

5.9.3 In vivo validation studies In vivo validation studies were performed in healthy individuals against anatomical landmarks and then against CT. Each in vivo study commenced with a pullback scan, systematically retracting the probe from the upper esophagus to the nasal cavity, to map the dimensions and anatomical landmarks of the pharynx. Each resulting data set was used to construct a movie of the pullback scan for convenient viewing and analysis. During the scan each subject was awake, supine and breathed quietly with the head maintained in a neutral position using a Shea headrest.

To validate the aOCT technique against a gold standard, simultaneous $a \mathrm{OCT}$ and CT images of the upper airway were obtained in five healthy subjects without obstructive sleep apnea (OSA). In each individual, a pullback scan data set was used to identify three sites for subsequent simultaneous scanning using $a \mathrm{OCT}$ and CT. Scans were obtained in each subject at the level of (1) the hypopharynx, $20 \mathrm{~mm}$ caudad to the tip of the epiglottis; (2) the oropharynx, just craniad to the tip of the epiglottis; and (3) the velopharynx (retropalatal pharynx), just craniad to the palatal rim. At each site, the $a \mathrm{OCT}$ probe was initially positioned and a lateral (scout) CT scan performed to precisely locate the probe. The gantry of the CT scanner was adjusted to scan through the tip of the probe orthogonal to the airway so that the $a \mathrm{OCT}$ and CT scan planes were coincident. Simultaneous aOCT and CT scans were then made while the subject held his/her breath at the end of normal exhalation (i.e., at functional residual capacity).

These studies were performed using the same CT scanner as the phantom validation studies. CT scout scans were performed to precisely locate the probe head $(80 \mathrm{kV}, 20 \mathrm{~mA})$ and low dose axial CT scans were used to capture cross- 
sectional images at specific sites in the upper airway $(80 \mathrm{kV}, 50 \mathrm{~mA})$.

5.9.4 References E1. Armstrong JJ, Leigh MS, Walton ID, Zvyagin AV, Alexandrov SA, Schwer S, Sampson DD, Hillman DR, Eastwood PR. In vivo size and shape measurement of the human upper airway using endoscopic long-range optical coherence tomography. Opt Expr 2003;11:1817-26.

E2. Sampson DD, Hillman TR. Optical coherence tomography. In: Palumbo G, Pratesi R, eds. Lasers and Current Optical Techniques in Biology. 1st ed. Cambridge, UK: Royal Society of Chemistry, 2004. p. 481-571.

E3. Bouma BE, Tearney GJ. Handbook of optical coherence tomography. New York: Marcel Dekker; 2002.

E4. Kwong KF, Yankelevich D, Chu KC, Heritage JP, Dienes A. 400-Hz Mechanical Scanning Optical Delay-Line. Opt Lett 1993;18:558-60.

E5. Tearney GJ, Bouma BE, Fujimoto JG. High-speed phase- and group-delay scanning with a grating-based phase control delay line. Opt Lett 1997;22:1811-3. E6. Zvyagin AV, Smith EDJ, Sampson DD. Delay and dispersion characteristics of a frequency-domain optical delay line for scanning interferometry. J Opt Soc Am A 2003;20:333-41.

E7. American National Standard for Safe Use of Lasers, ANSI Z136.1-2000. Orlando: Laser Institute of America; 2000. 


\section{Conclusions}

\subsection{Discussion of outcomes}

The main outcome of this thesis is the successful demonstration of the first system for taking quantitative measurements in the human upper airway. The system met the initial specifications for airway measurements - the image size of $52 \times 52 \mathrm{~mm}$, rotation rate of $1.25 \mathrm{~Hz}$, resolution better than $0.5 \mathrm{~mm}$, catheter size of $3 \mathrm{~mm}$, and recording time sufficient for an overnight sleep experiment, all satisfied the requirements. Validation experiments have been carried out and published demonstrating agreement with an OSA-research gold standard measurement modality, CT.

During the creation of the aOCT system the main areas of engineering and design were the optical design and fabrication process of the optical probes, the modelling and analysis of the long-range frequency-domain optical delay line, the electronic subsystems (signal processing and probe control system), the software design, the data processing and presentation, the development of the experimental protocols in deploying the system in the clinical setting of a sleep laboratory.

Quantitative airway shape measurements have been taken repeatedly and over extended periods on over 40 subjects in a normal sleep laboratory, which was previously not possible. We have shown that clinically relevant parameters such as airway compliance can be measured over large numbers of subjects, in the sleep laboratory. A sleep experiment has been conducted which has shown that the system is compatible with a full polysomnography setup and can provide synchronized measurements in the airway during normal sleep and apnoea events.

\subsection{Future work}

The two main areas of future work for this project are in clinical measurements using aOCT and improvements to the system. There is a large scope for clinical experiments with $a \mathrm{OCT}$, some of which are currently underway. There is a large amount of medical research which has looked at airway structure and dynamic airway behaviour in a variety of situations. These have either been nonquantitative, carried out during wakefulness, or involved unquantified/unconfirmed sleep. aOCT has now created the possibility to revisit all of these experiments during sleep, in a normal sleep laboratory, with polysomnography equipment 
used to quantify sleep state and the aOCT instrument measuring accurate airway structure. The initial set of experiments reported in this thesis have looked at validation of the technology and initial exploration of the capability for making meaningful comparisons between the airways of apnoea patients and nonapnoeic controls. These experiments are presently ongoing.

Another area of research which would be highly suitable for aOCT application is airway patency during anaesthesia. It has been found that the occurance of airway collapse during anesthesia and a history of obstructive sleep apnoea are correlated [142]. Imaging the airway in this state using aOCT would allow this link to investigated further, in a normal operating theatre - rather than in a CT or MRI imaging suite, which may not even be feasible. Under anaesthesia muscle activity is highly suppressed, therefore airway behaviour could be studied with $a \mathrm{OCT}$ while the dynamics of collapse are decoupled from compensatory pharyngeal muscle reflexes.

Other future work is in technical improvements to the aOCT system. Increased rotation rate, distance range, and sensitivity would improve the quality of the data. There are relatively straightforward paths to implementing these changes and these are currently in progress. During the last few years technology in fibre-optic rotary joints and galvanometers has improved - these can be incorporated directly into the system to upgrade the performance. More powerful light sources suited to $a \mathrm{OCT}$ have become available at lower cost, and indeed during the course of this project the light source has been upgraded (for a lower price than the original). The recognition of the many advantages of frequencydomain optical coherence tomography has prompted the development of several commerically-available tunable light sources, which will enable the creation of swept-source OCT systems without the necessity of building a custom tunable laser. Therefore it is logical that the aOCT system should eventually use frequency-domain OCT rather than time-domain OCT.

The catheter, probe and its drive system has the potential for improvement in technology. This includes custom-fabrication of a catheter for a reduced outer diameter, improved mechanical properties, and a smoother surface. Micro-striations causing beam distortion could be eliminated by careful attention to the fabrication process. Mechanical properties could be addressed to reduce the slight inherent bend in the catheter tubing which could potentially cause difficulties during nasal insertion. Also, a harder material might allow the catheter outer diameter to be reduced without increasing collapsibility of the tubing. A significant modifica- 
tion of the probe would be a change to a design in which the rotational scanning is performed by a micro-motor in the probe itself. This would eliminate the need for a fibre-optic rotary joint and for the whole probe to rotate, reducing the likelihood of non-uniform rotation distortion in the $a$ OCT images. It would also mean that the probe downlead to the patient could be made more robust, an advantage for measurements performed during sleep if the patient should toss and turn during the night. The smallest currently available micro-motor is slightly too large (1.9 mm diameter) for use in the $a \mathrm{OCT}$ system; however, it is likely that motors of smaller dimensions will be developed.

The processing and analysis of aOCT data remains an area with much potential for further development. The procedure of manual tracing of the airway contours is time-consuming and tedious for large data sets. The implementation of semi- or fully-automated contour tracing would save time and could increase accuracy by eliminating possible researcher bias. Also, automatic contour location would simplify the process of three-dimensional reconstruction. 



\section{Bibliography}

[1] Terry Young, Paul E. Peppard, and Daniel J. Gottlieb. Epidemiology of obstructive sleep apnea: A population health perspective. American Journal of Respiratory and Critical Care Medicine, 165(9):1217-1239, 2002.

[2] A. Rechtschaffen and B. M. Bergmann. Sleep deprivation in the rat by the disk-over-water method. Behavioural Brain Research, 69(1-2):55-63, 1995.

[3] J. J. Pilcher and A. I. Huffcutt. Effects of sleep deprivation on performance: a meta-analysis. Sleep, 19(4):318-26, 1996.

[4] R. M. Benca and J. Quintas. Sleep and host defenses: a review. Sleep, 20(11):1027-37, 1997.

[5] J He, MH Kryger, FJ Zorick, W Conway, and T Roth. Mortality and apnea index in obstructive sleep apnea. experience in 385 male patients. Chest, 94(1):9-14, 1988.

[6] M. Partinen, A. Jamieson, and C. Guilleminault. Long-term outcome for obstructive sleep apnea syndrome patients. mortality.[see comment]. Chest, 94(6):1200-4, 1988. Comment in: Chest. 1989 Sep;96(3):703-4; PMID: 2766844.

[7] Paul E. Peppard, Terry Young, Mari Palta, and James Skatrud. Prospective study of the association between sleep-disordered breathing and hypertension. New England Journal of Medicine, 342(19):1378-1384, 2000.

[8] C F P George. Reduction in motor vehicle collisions following treatment of sleep apnoea with nasal CPAP. Thorax, 56(7):508-512, 2001.

[9] T. Young, J. Blustein, L. Finn, and M. Palta. Sleep-disordered breathing and motor vehicle accidents in a population-based sample of employed adults. Sleep, 20(8):608-13, 1997.

[10] P.V. Tishler, S. Redline, V. Ferrette, M.G. Hans, and M.D. Altose. The association of sudden unexpected infant death with obstructive sleep apnea. American Journal of Respiratory and Critical Care Medicine, 153(6 I):1857-1863, 1996. 
[11] David Gozal. Sleep-disordered breathing and school performance in children 10.1542/peds.102.3.616. Pediatrics, 102(3):616-620, 1998.

[12] Access Economics. Wake up australia: The value of healthy sleep. Technical report, June 2005.

[13] A. Sassani, L. J. Findley, M. Kryger, E. Goldlust, C. George, and T. M. Davidson. Reducing motor-vehicle collisions, costs, and fatalities by treating obstructive sleep apnea syndrome.[see comment]. Sleep, 27(3):453-8, 2004. Comment in: Sleep. 2004 May 1;27(3):369-70; PMID: 15164885.

[14] Jamil L. Hossain and Colin M. Shapiro. The prevalence, cost implications, and management of sleep disorders: An overview. Sleep and Breathing, 6(2):85-102, 2002.

[15] Terry Young, Mari Palta, Jerome Dempsey, James Skatrud, Steven Weber, and Safwan Badr. The occurrence of sleep-disordered breathing among middle-aged adults. New England Journal of Medicine, 328(17):1230-1235, 1993.

[16] E. O. Bixler, A. N. Vgontzas, T. Ten Have, K. Tyson, and A. Kales. Effects of age on sleep apnea in men: I. prevalence and severity. American Journal of Respiratory and Critical Care Medicine, 157(1):144-8, 1998.

[17] J. Duran, S. Esnaola, R. Rubio, and A. Iztueta. Obstructive sleep apneahypopnea and related clinical features in a population-based sample of subjects aged 30 to 70 yr. American Journal of Respiratory and Critical Care Medicine, 163(3 Pt 1):685-9, 2001.

[18] H Bearpark, L Elliott, R Grunstein, S Cullen, H Schneider, W Althaus, and C Sullivan. Snoring and sleep apnea. a population study in australian men. American Journal of Respiratory and Critical Care Medicine, 151(5):1459-1465, 1995.

[19] Taskforce. Sleep-related breathing disorders in adults: recommendations for syndrome definition and measurement techniques in clinical research. the report of an american academy of sleep medicine task force. Sleep, 22(5):667-89, 1999. 
[20] Australian Health Technology Advisory Committee. The effectiveness and cost effectiveness of nasal continuous positive airway pressure in the treatment of obstructive sleep apnoea in adults. Technical report, Government Public Services, 1996.

[21] C F Ryan and L L Love. Unpredictable results of laser assisted uvulopalatoplasty in the treatment of obstructive sleep apnoea. Thorax, 55(5):399-404, 2000.

[22] S. Fujita, W. Conway, F. Zorick, and T. Roth. Surgical correction of anatomic abnormalities in obstructive sleep apnea syndrome: uvulopalatopharyngoplasty. Otolaryngology - Head E Neck Surgery, 89(6):923-34, 1981.

[23] K. B. Schechtman, A. E. Sher, and J. F. Piccirillo. Methodological and statistical problems in sleep apnea research: the literature on uvulopalatopharyngoplasty. Sleep, 18(8):659-66, 1995.

[24] I. Marshall, N. J. Maran, S. Martin, M. A. Jan, J. E. Rimmington, J. J. Best, G. B. Drummond, and N. J. Douglas. Acoustic reflectometry for airway measurements in man: implementation and validation. Physiological Measurement, 14(2):157-69, 1993.

[25] R. Chaban, P. Cole, and V. Hoffstein. Site of upper airway obstruction in patients with idiopathic obstructive sleep apnea. Laryngoscope, 98(6 Pt 1):641$7,1988$.

[26] D. W. Hudgel, M. Mulholland, and C. Hendricks. Neuromuscular and mechanical responses to inspiratory resistive loading during sleep. Journal of Applied Physiology, 63(2):603-8, 1987.

[27] V Hoffstein and JJ Fredberg. The acoustic reflection technique for noninvasive assessment of upper airway area. European Respiratory Journal, 4(5):602-611, 1991.

[28] M. S. Badr, F. Toiber, J. B. Skatrud, and J. Dempsey. Pharyngeal narrowing occlusion during central sleep-apnea. Journal of Applied Physiology, 78(5):1806-1815, 1995.

[29] E. F. Haponik, P. L. Smith, M. E. Bohlman, R. P. Allen, S. M. Goldman, and E. R. Bleecker. Computerized tomography in obstructive sleep apnea. 
correlation of airway size with physiology during sleep and wakefulness. American Journal of Respiratory and Critical Care Medicine, 127(2):221-6, 1983.

[30] Jr. Shepard, J. W., M. Garrison, and W. Vas. Upper airway distensibility and collapsibility in patients with obstructive sleep apnea.[see comment]. Chest, 98(1):84-91, 1990. Comment in: Chest. 1991 Jul;100(1):290-1; PMID: 2060377.

[31] A. A. Lowe, J. A. Fleetham, S. Adachi, and C. F. Ryan. Cephalometric and computed tomographic predictors of obstructive sleep apnea severity.[see comment]. American Journal of Orthodontics $\mathcal{E}$ Dentofacial Orthopedics, 107(6):589-95, 1995. Comment in: Am J Orthod Dentofacial Orthop. 1995 Nov;108(5):13A; PMID: 7484957.

[32] J. R. Wheatley, W. T. Kelly, A. Tully, and L. A. Engel. Pressure-diameter relationships of the upper airway in awake supine subjects. Journal of Applied Physiology, 70(5):2242-51, 1991.

[33] R. Arens, S. Sin, J. M. McDonough, J. M. Palmer, T. Dominguez, H. Meyer, D. M. Wootton, and A. I. Pack. Changes in upper airway size during tidal breathing in children with obstructive sleep apnea syndrome. American Journal of Respiratory and Critical Care Medicine, 171(11):1298-304, 2005.

[34] Richard J. Schwab, Michael Pasirstein, Robert Pierson, Adonna Mackley, Robert Hachadoorian, Raanan Arens, Greg Maislin, and Allan I. Pack. Identification of upper airway anatomic risk factors for obstructive sleep apnea with volumetric magnetic resonance imaging. American Journal of Respiratory and Critical Care Medicine, 168(5):522-530, 2003.

[35] Anil N. Rama, Shivan H. Tekwani, and Clete A. Kushida. Sites of obstruction in obstructive sleep apnea. Chest, 122(4):1139-1147, 2002.

[36] Raanan Arens, Joseph M. McDonough, Aaron M. Corbin, Nathania K. Rubin, Mary Ellen Carroll, Allan I. Pack, Jianguo Liu, and Jayaram K. Udupa. Upper airway size analysis by magnetic resonance imaging of children with obstructive sleep apnea syndrome. American Journal of Respiratory and Critical Care Medicine, 167(1):65-70, 2003.

[37] R. F. Fregosi, S. F. Quan, K. L. Kaemingk, W. J. Morgan, J. L. Goodwin, R. Cabrera, and A. Gmitro. Sleep-disordered breathing, pharyngeal size and soft tissue anatomy in children. J Appl Physiol, 95(5):2030-2038, 2003. 
[38] Jr. Shepard, J. W., W. B. Gefter, C. Guilleminault, E. A. Hoffman, V. Hoffstein, D. W. Hudgel, P. M. Suratt, and D. P. White. Evaluation of the upper airway in patients with obstructive sleep apnea. Sleep, 14(4):361-71, 1991.

[39] B. T. Woodson and M. R. Wooten. Comparison of upper-airway evaluations during wakefulness and sleep. Laryngoscope, 104(7):821-8, 1994.

[40] G. P. Katsantonis, C. S. Maas, and J. K. Walsh. The predictive efficacy of the Muller maneuver in uvulopalatopharyngoplasty. Laryngoscope, 99(7 Pt 1):677-80, 1989.

[41] R. Conradt, Brandenburg U., Cassell W., Juhasz J., and Peter J. H. Oesophageal pressure measurements - interference with sleep structure in nonapnoeic snorers. European Respiratory Journal, 8:255s, 1995.

[42] O. Skatvedt, H. Akre, and O. B. Godtlibsen. Nocturnal polysomnography with and without continuous pharyngeal and esophageal pressure measurements. Sleep., 19(6):485-90, 1996.

[43] Clemens von Birgelen, Evelyn A. de Vrey, Gary S. Mintz, Antonino Nicosia, Nico Bruining, Wenguang Li, Cornelis J. Slager, Jos R. T. C. Roelandt, Patrick W. Serruys, and Pim J. de Feyter. Ecg-gated three-dimensional intravascular ultrasound : Feasibility and reproducibility of the automated analysis of coronary lumen and atherosclerotic plaque dimensions in humans. Circulation, 96(9):2944-2952, 1997.

[44] M. H. Chan, W. M. Lin, C. H. Zhou, and J. N. Y. Qu. Miniaturized threedimensional endoscopic imaging system based on active stereovision. Applied Optics, 42(10):1888-1898, 2003.

[45] S. Donati, G. Giuliani, and S. Merlo. Laser-diode feedback interferometer for measurement of displacements without ambiguity. IEEE Journal of Quantum Electronics, 31(1):113-119, 1995.

[46] P. J. de Groot, G. M. Gallatin, and S. H. Macomber. Ranging and velocimetry signal generation in a backscatter-modulated laser diode. Applied Optics, 27(21):4475-4480, 1987.

[47] F. Gouaux, N. Servagent, and T. Bosch. Absolute distance measurement with an optical feedback interferometer. Applied Optics, 37(28):6684-6689, 1998. 
[48] S. F. Collins, W. X. Huang, M. M. Murphy, K. T. V. Grattan, and A. W. Palmer. A simple laser-diode ranging scheme using an intensity-modulated fmcw approach. Measurement Science E Technology, 4(12):1437-1439, 1993.

[49] B. Journet and G. Bazin. A low-cost laser range finder based on an FMCW-like method. IEEE Transactions on Instrumentation and Measurement, 49(4):840-843, 2000.

[50] Barry L. Stann, William C. Ruff, and Zoltan G. Sztankay. Intensitymodulated diode laser radar using frequency-modulation/continuouswave ranging techniques. Optical Engineering, 35(11):3270-3278, 1996.

[51] A. A. Michelson and J. R. Benoit. Dtermination exprimentale de la valeur du mtre en longueurs d'ondes lumineuses. Trav. Mem. Bur. Int. Poids Mes., 11:1, 1895.

[52] Charles R. Tilford. Analytical procedure for determining lengths from fractional fringes. Applied Optics, 16(7):1857-1860, 1977.

[53] R. C. Youngquist, S. Carr, and D. E. N. Davies. Optical coherence-domain reflectometry - a new optical evaluation technique. Optics Letters, 12(3):158160, 1987.

[54] K. Takada, I. Yokohama, K. Chida, and J. Noda. New measurement system for fault location in optical wave-guide devices based on an interferometrictechnique. Applied Optics, 26(9):1603-1606, 1987.

[55] B. L. Danielson and C. D. Whittenberg. Guided-wave reflectometry with micrometer resolution. Applied Optics, 26(14):2836-2842, 1987.

[56] David Huang, Eric A. Swanson, Charles P. Lin, Joel S. Schuman, William G. Stinson, Warren Chang, Michael R. Hee, Thomas Flotte, Kenton Gregory, Carmen A. Puliafito, and James G. Fujimoto. Optical coherence tomography. Science, 254(5035):1178-1181, 1991.

[57] W. Drexler, U. Morgner, R. K. Ghanta, F. X. Krtner, J. S. Schuman, and J. G. Fujimoto. Ultrahigh-resolution ophthalmic optical coherence tomography. Nature Medicine, 7(4):502-507, 2001.

[58] J. G. Fujimoto, S. A. Boppart, G. J. Tearney, B. E. Bouma, C. Pitris, and M. E. Brezinski. High resolution in vivo intra-arterial imaging with optical coherence tomography. Heart, 82(2):128-133, 1999. 
[59] F. G. Bechara, T. Gambichler, M. Stucker, A. Orlikov, S. Rotterdam, P. Altmeyer, and K. Hoffmann. Histomorphologic correlation with routine histology and optical coherence tomography. Skin Research and Technology, 10(3):169-73, 2004.

[60] V.X.D. Yang, M.L. Gordon, B. Qi, E.S. Yue, S. Bisland, J. Pekar, S. Lo, B.C. Wilson, I.A. Vitkin, S. Tang, and N. Marcon. High sensitivity detection and monitoring of microcirculation using cutaneous and catheter probes for doppler optical coherence tomography. Proceedings of SPIE - The International Society for Optical Engineering, 4965:153-159, 2003.

[61] B. E. Bouma, G. J. Tearney, C. C. Compton, and N. S. Nishioka. Highresolution imaging of the human esophagus and stomach in vivo using optical coherence tomography. Gastrointestinal Endoscopy, 51(4):467-474, 2000.

[62] S. Jackle, N. Gladkova, F. Feldchtein, A. Terentieva, B. Brand, G. Gelikonov, V. Gelikonov, A. Sergeev, A. Fritscher-Ravens, J. Freund, U. Seitz, S. Schroder, and N. Soehendra. In vivo endoscopic optical coherence tomography of the human gastrointestinal tract - toward optical biopsy. Endoscopy, 32(10):743-749, 2000.

[63] X. D. Li, S. A. Boppart, J. Van Dam, H. Mashimo, M. Mutinga, W. Drexler, M. Klein, C. Pitris, M. L. Krinsky, M. E. Brezinski, and J. G. Fujimoto. Optical coherence tomography: advanced technology for the endoscopic imaging of barrett's esophagus. Endoscopy, 32(12):921-30, 2000.

[64] C. Pitris, C. Jesser, S. A. Boppart, D. Stamper, M. E. Brezinski, and J. G. Fujimoto. Feasibility of optical coherence tomography for high-resolution imaging of human gastrointestinal tract malignancies. Journal of Gastroenterology, 35(2):87-92, 2000.

[65] A. R. Tumlinson, L. P. Hariri, U. Utzinger, and J. K. Barton. Miniature endoscope for simultaneous optical coherence tomography and laser-induced fluorescence measurement. Applied Optics, 43(1):113-121, 2004.

[66] Y.T. Pan, T.Q. Xie, C.W. Du, S. Bastacky, S. Meyers, and M.L. Zeidel. Enhancing early bladder cancer detection with fluorescence-guided endoscopic optical coherence tomography. Optics Letters, 28(24):2485-2487, 2003. 
[67] F. I. Feldchtein, G. V. Gelikonov, V. M. Gelikonov, R. R. Iksanov, R. V. Kuranov, A. M. Sergeev, N. D. Gladkova, M. N. Ourutina, J. A. Warren, and D. H. Reitze. In vivo OCT imaging of hard and soft tissue of the oral cavity. Optics Express, 3(6):239-250, 1998.

[68] Andrei Shakhov, Anna Terentjeva, Natalia Gladkova, Ludmila Snopova, Yuri Chumakov, Felix Feldchtein, Valentin Gelikonov, Grigory Gelikonov, and Alexander Sergeev. Capabilities of optical coherence tomography in laryngology. Proceedings of SPIE - The International Society for Optical Engineering Proceedings of the 1999 Lasers in Surgery: Advanced Characterization, Therapeutics, and Systems IX, Jan 23-Jan 24 1999, 3590:250-260, 1999.

[69] Ying Yang, Suzanne Whiteman, Daniel Gey van Pittius, Yonghong He, Ruikang K Wang, and Monica A Spiteri. Use of optical coherence tomography in delineating airways microstructure: comparison of OCT images to histopathological sections. Physics in Medicine and Biology, 49(7):1247, 2004.

[70] G.J. Tearney, S.A. Boppart, B.E. Bouma, C. Pitris, M.E. Brezinski, J.F. Southern, E.A. Swanson, and J.G. Fujimoto. High speed catheter/endoscopic optical coherence tomography for the optical biopsy of in vivo tissues. In Proceedings of the 1997 Conference on Lasers and Electro-Optics, CLEO,May 18-23 1997, volume 11 of Conference on Lasers and Electro-Optics Europe - Technical Digest, page 211, Baltimore,MD,USA, 1997. IEEE,Piscataway,NJ,USA.

[71] B. E. Bouma and G. J. Tearney. Power-efficient nonreciprocal interferometer and linear-scanning fiber-optic catheter for optical coherence tomography. Optics Letters, 24(8):531-533, 1999.

[72] S.A. Boppart, B.E. Bouma, C. Pitris, G.J. Tearney, J.G. Fujimoto, and M.E. Brezinski. Forward-imaging instruments for optical coherence tomography. Optics Letters, 22(21):1618, 1997.

[73] J. G. Fujimoto. Optical coherence tomography for ultrahigh resolution in vivo imaging. Nature Biotechnology, 21(11):1361-1367, 2003.

[74] Julian J. Armstrong, Matthew S. Leigh, Thomas Gutzler, David R. Hillman, Peter R. Eastwood, and David D. Sampson. Hollow organ profiling system based on endoscopic optical coherence tomography. In PEECS2003: The Fourth Postgraduate Electrical Engineering and Computing Symposium, pages 121-126, Perth, Australia, 2003. Murdoch University, Australia. 
[75] David D. Sampson and Timothy. R. Hillman. Optical coherence tomography. In Giuseppe Palumbo and Riccardo Pratesi, editors, Lasers and Current Optical Techniques in Biology, volume 4 of Comprehensive Series in Photochemistry and Photobiology, pages 481-571. Royal Society of Chemistry, Cambridge, UK, 1st edition, 2004.

[76] M.A. Ciscar, G. Juan, V. Martinez, M. Ramon, T. Lloret, J. Minguez, M. Armengot, J. Marin, and J. Basterra. Magnetic resonance imaging of the pharynx in osa patients and healthy subjects. European Respiratory Journal, 17(1):79-86, 2001.

[77] FrankJ. Trudo, WarrenB. Gefter, KevinC. Welch, KrishanuB. Gupta, Greg Maislin, and RichardJ. Schwab. State-related changes in upper airway caliber and surrounding soft-tissue structures in normal subjects. American Journal of Respiratory and Critical Care Medicine, 158(4):1259-1270, 1998.

[78] C. Froehly, B. Colombeau, and M. Vampouille. Shaping and analysis of picosecond light-pulses. Progress in Optics, 20:65-153, 1983.

[79] J. P. Heritage, A. M. Weiner, and R. N. Thurston. Picosecond pulse shaping by spectral phase and amplitude manipulation. Optics Letters, 10(12):609611, 1985.

[80] K. F. Kwong, D. Yankelevich, K. C. Chu, J. P. Heritage, and A. Dienes. 400hz mechanical scanning optical delay-line. Optics Letters, 18(7):558-560, 1993.

[81] Andrew M. Rollins, Manish Kulkarni, Siavash Yazdanfar, and Joseph A. Izatt. Video rate optical coherence tomography. volume 21 of OSA Trends in Optics and Photonics Series, page 353, 1998.

[82] K.K.M.B.D. Silva, A.V. Zvyagin, and D.D. Sampson. Extended range, rapid scanning optical delay line for biomedical interferometric imaging. Electronics Letters, 35(17):1404-1406, 1999.

[83] N. V. Iftimia, B. E. Bouma, J. F. de Boer, B. H. Park, B. Cense, and G. J. Tearney. Adaptive ranging for optical coherence tomography. Optics Express, 12(17):4025-4034, 2004. 
[84] Y. H. Zhao, Z. P. Chen, C. Saxer, S. H. Xiang, J. F. de Boer, and J. S. Nelson. Phase-resolved optical coherence tomography and optical doppler tomography for imaging blood flow in human skin with fast scanning speed and high velocity sensitivity. Optics Letters, 25(2):114-116, 2000.

[85] J. F. de Boer, C. E. Saxer, and J. S. Nelson. Stable carrier generation and phase-resolved digital data processing in optical coherence tomography. Applied Optics, 40(31):5787-5790, 2001.

[86] Andrew M. Rollins, Siavash Yazdanfar, Jennifer K. Barton, and Joseph A. Izatt. Real-time in vivo color doppler optical coherence tomography. Journal of Biomedical Optics, 7(1):123-129, 2002.

[87] G. J. Tearney, B. E. Bouma, and J. G. Fujimoto. High-speed phase- and group-delay scanning with a grating-based phase control delay line. Optics Letters, 22(23):1811-1813, 1997.

[88] Andrew M. Rollins, Manish D. Kulkarni, Siavash Yazdanfar, Rujchai Ungarunyawee, and Joseph A. Izatt. In vivo video rate optical coherence tomography. Optics Express, 3(6):219-228, 1998.

[89] A. V. Zvyagin, E. D. J. Smith, and D. D. Sampson. Delay and dispersion characteristics of a frequency-domain optical delay line for scanning interferometry. Journal of the Optical Society of America A-Optics, Image Science, and Vision, 20(2):333-341, 2003.

[90] D. M. Baney and W. V. Sorin. Extended-range optical low-coherence reflectometry using a recirculating delay technique. IEEE Photonics Technology Letters, 5(9):1109-1112, 1993.

[91] E.A. Swanson, D. Huang, M.R. Hee, J.G. Fujimoto, C.P. Lin, and C.A. Puliafito. High-speed optical coherence domain reflectometry. Optics Letters, 17(2):151-153, 1992.

[92] G.J. Tearney, S.A. Boppart, B. Bouma, M.E. Brezinski, B. Golubovic, J.F. Southern, E.A. Swanson, and J.G. Fujimoto. Single-mode fiber-optic catheter/endoscope for optical coherence tomography. Pacific Rim Conference on Lasers and Electro-Optics, CLEO - Technical Digest Proceedings of the 1996 Conference on Lasers and Electro-Optics, CLEO'96, Jun 2-7 1996, pages 57-58, 1996. 
[93] C. B. Su. Achieving variation of the optical path length by a few millimeters at millisecond rates for imaging of turbid media and optical interferometry: A new technique. Optics Letters, 22(10):665-667, 1997.

[94] J. Ballif, R. Gianotti, Ph. Chavanne, R. Walti, and R.P. Salathe. Rapid and scalable scans at $21 \mathrm{~m} / \mathrm{s}$ in optical low-coherence reflectometry. Optics Letters, 22(11):757-759, 1997.

[95] N. Delachenal, R. Gianotti, R. Walti, H. Limberger, and R. P. Salathe. Constant high-speed optical low-coherence reflectometry over $0.12 \mathrm{~m}$ scan range. Electronics Letters, 33(24):2059-2061, 1997.

[96] J. Szydlo, N. Delachenal, R. Giannotti, R. Walti, H. Bleuler, and R. P. Salathe. Air-turbine driven optical low-coherence reflectometry at 28.6-khz scan repetition rate. Optics Communications, 154(1-3):1-4, 1998.

[97] L. Giniunas, R. Danielius, and R. Karkockas. Scanning delay line with a rotating-parallelogram prism for low-coherence interferometry. Applied $\mathrm{Op}$ tics, 38(34):7076-7079, 1999.

[98] M. Lai. Kilohertz scanning optical delay line employing a prism array. Applied Optics, 40(34):6334-6336, 2001.

[99] Nan Guang Chen and Quing Zhu. Rotary mirror array for high-speed optical coherence tomography. Optics Letters, 27(8 Apr 15):607-609, 2002.

[100] T. Shiina, Y. Moritani, M. Ito, and Y. Okamura. Long-optical-path scanning mechanism for optical coherence tomography. Applied Optics, 42(19):37953799, 2003.

[101] J. H. Liu, D. S. Wu, and K. Y. Tseng. Fabrication and characterization of grin plastic rods containing silver nanoparticles with novel surfmers. Macromolecular Chemistry and Physics, 205(16):2205-2213, 2004.

[102] Christopher E. Saxer, Johannes F. de Boer, B. Hyle Park, Yonghua Zhao, Zhongping Chen, and J. Stuart Nelson. Fiber-based polarization-sensitive optical coherence tomography. In Coherence Domain Optical Methods in Biomedical Science and Clinical Applications IV, Jan 24-Jan 26 2000, volume 3915 of Proceedings of SPIE - The International Society for Optical Engineering, pages 68-75, San Jose, CA, USA, 2000. Society of Photo-Optical Instrumentation Engineers, Bellingham, WA, USA. 
[103] Z. H. Ding, G. Peavy, H. W. Ren, Y. H. Zhao, W. G. Jung, J. J. Miao, J. S. Nelson, and Z. P. Chen. Phase resolved functional optical coherence tomography: Simultaneous imaging of tissue structure, blood flow and birefringence. Lasers in Surgery and Medicine, pages 5-5, 2002.

[104] M. C. Pierce, B. H. Park, B. Cense, and J. F. de Boer. Simultaneous intensity, birefringence, and flow measurements with high-speed fiber-based optical coherence tomography. Optics Letters, 27(17):1534-1536, 2002.

[105] R. E. Wagner and W. J. Tomlinson. Coupling efficiency of optics in singlemode fiber components. Applied Optics, 21(15):2671-2688, 1982.

[106] David D. Sampson, Andrei V. Zvyagin, Elwyn D. J. Smith, Ian D. Walton, Julian J. Armstrong, and K. K. M. B. D. Silva. The frequency-domain delay line in optical coherence tomography design, capabilities, and limitations (invited). In Conference on Lasers and Electrooptics-Pacific Rim (CLEOPACRIM), Taiwan, 2003.

[107] Joseph W. Goodman. Statistical Optics. John Wiley \& Sons, New York, 2000.

[108] M. Nazarathy, W.V. Sorin, D.M. Baney, and S.A. Newton. Spectral analysis of optical mixing measurements. Journal of Lightwave Technology, 7(7):10831096, 1989.

[109] H. Hodara. Statistics of thermal and laser radiation. Proceedings of the IEEE, 53(7):696-704, 1965.

[110] P.R. Morkel, R.I. Laming, and D.N. Payne. Noise characteristics of highpower doped-fibre superluminescent sources, 1990.

[111] K. Takada. Noise in optical low-coherence reflectometry. IEEE Journal of Quantum Electronics, 34(7):1098-1108, 1998.

[112] Govind P. Agrawal. Fiber-Optic Communication Systems. John Wiley \& Sons, 1992.

[113] AS/NZ. Australian/New Zealeand standard: Safety of laser products, AS/NZ 2211.1:2004. Technical report, Standards Australia/Standards New Zealand, 2004.

[114] ANSI. American National Standard for Safe Use of Lasers, ANSI Z136.1-2000. Laser Institute of America, Orlando, 2000. 
[115] Andrew M. Rollins and Joseph A. Izatt. Optimal interferometer designs for optical coherence tomography. Optics Letters, 24(21):1484, 1999.

[116] M. R. Hee. Optical coherence tomography: Theory. In B. E. Bouma and G. J. Tearney, editors, Handbook of Optical Coherence Tomography, pages 4166. Marcel Dekker, Inc., New York, 2001.

[117] M. A. Choma, M. V. Sarunic, C. H. Yang, and J. A. Izatt. Sensitivity advantage of swept source and fourier domain optical coherence tomography. Optics Express, 11(18):2183-2189, 2003.

[118] R. Leitgeb, C. K. Hitzenberger, and A. F. Fercher. Performance of fourier domain vs. time domain optical coherence tomography. Optics Express, 11(8):889-894, 2003.

[119] E. Gotzinger, M. Pircher, R. A. Leitgeb, and C. K. Hitzenberger. High speed full range complex spectral domain optical coherence tomography. Optics Express, 13(2):583-594, 2005.

[120] N. A. Nassif, B. Cense, B. H. Park, M. C. Pierce, S. H. Yun, B. E. Bouma, G. J. Tearney, T. C. Chen, and J. F. de Boer. In vivo high-resolution video-rate spectral-domain optical coherence tomography of the human retina and optic nerve. Optics Express, 12(3):367-376, 2004.

[121] S. H. Yun, G. J. Tearney, J. F. de Boer, and B. E. Bouma. Motion artifacts in optical coherence tomography with frequency-domain ranging. Optics Express, 12(13):2977-2998, 2004.

[122] J. J. Armstrong and D. D. Sampson. Distance ranging to biological tissue using fibre-optic fabry-perot, short tuning range FMCW interferometry. In H. C. Lefevre A. G. Mignani, editor, 14th International Conference on Optical Fibre Sensors, volume 4185, pages 366-369, Florence, Italy, 2000. SPIE.

[123] D. Marcuse. Theory of Dielectric Waveguides. Academic Press, New York, 1974.

[124] M. Gu, C. J. R. Sheppard, and X. Gan. Image-formation in a fiberoptic confocal scanning microscope. Journal of the Optical Society of America A-Optics, Image Science, and Vision, 8(11):1755-1761, 1991.

[125] SELFOC Product Guide. NSG America, 2001. 
[126] Bahaa E. A. Saleh and Malvin Carl Teich. Fundamentals of Photonics. Wiley, New York, 2nd edition, 1991.

[127] Stefan Schwer. Optomechanics and Microoptics for an Endoscopic OCT System. PhD thesis, 2002.

[128] E. Verdonk, P. Webb, and M. Greenstein. Rotary encoding for intravascular ultrasonic imaging systems. Ultrasonics, Ferroelectrics and Frequency Control, IEEE Transactions on, 47(6):1482-1493, 2000.

[129] K. K. M. B. D. Silva. Optical Coherence Tomography: Technology Enhancements and Novel Applications. PhD thesis, University of Western Australia, 2003.

[130] Polysomnography Task Force of the American Sleep Disorders Association Standards of Practice Committee. Practice parameters for the indications for polysomnography and related procedures. Sleep, 20(6):406-22, 1997.

[131] B. H. Park, M. C. Pierce, B. Cense, and J. F. de Boer. Real-time multifunctional optical coherence tomography. Optics Express, 11(7):782-793, 2003.

[132] RonaldL. Arenson, DevP. Chakraborty, SridharB. Seshadri, and HaroldL. Kundel. The digital imaging workstation. Journal of Digital Imaging, 16(1):142-162, 2003.

[133] J. Rogowska, C. M. Bryant, and M. E. Brezinski. Cartilage thickness measurements from optical coherence tomography. Journal of the Optical Society of America A- Optics, Image Science, and Vision., 20(2):357-67, 2003.

[134] Y. K. Lee and W. T. Rhodes. Nonlinear image-processing by a rotating kernel transformation. Optics Letters, 15(23):1383-1385, 1990.

[135] D. Koozekanani, K. Boyer, and C. Roberts. Retinal thickness measurements from optical coherence tomography using a markov boundary model. IEEE Transactions on Medical Imaging, 20(9):900-916, 2001.

[136] M. Kass, A. Witkin, and D. Terzopoulos. Snakes - active contour models. International Journal of Computer Vision, 1(4):321-331, 1987.

[137] Chenyang $\mathrm{Xu}$ and J.L. Prince. Snakes, shapes, and gradient vector flow. IEEE Transactions on Image Processing, 7(3):359-369, 1998. 
[138] Thomas Gutzler. 3D-Visualisation of OCT-images. PhD thesis, University of Western Australia, 2003.

[139] F. A. Jolesz, W. E. Lorensen, H. Shinmoto, H. Atsumi, S. Nakajima, P. Kavanaugh, P. Saiviroonporn, S. E. Seltzer, S. G. Silverman, M. Phillips, and R. Kikinis. Interactive virtual endoscopy. American Journal of Roentgenology, 169(5):1229-1235, 1997.

[140] K. Neumann, J. Winterer, M. Kimmig, D. Burger, A. Einert, K. H. Allmann, M. Hauer, and M. Langer. Real-time interactive virtual endoscopy of the tracheo-bronchial system: influence of ct imaging protocols and observer ability. European Journal of Radiology, 33(1):50-54, 2000.

[141] A. Wahle, M. E. Olszewski, and M. Sonka. Interactive virtual endoscopy in coronary arteries based on multimodality fusion. IEEE Transactions on Medical Imaging, 23(11):1391-1403, 2004.

[142] D. R. Hillman, P. R. Platt, and P. R. Eastwood. The upper airway during anaesthesia. British Journal of Anaesthesia, 91(1):31-39, 2003.

[143] Simon Charles Moore. A theoretical framework for new approaches to scanning and acquisition in optical coherence tomography. PhD thesis, University of Western Australia, 2002.

[144] E. D. J. Smith, A. V. Zvyagin, and D. D. Sampson. Real-time dispersion compensation in scanning interferometry. Optics Letters, 27(22):1998-2000, 2002.

[145] A. V. Zvyagin and D. D. Sampson. Achromatic optical phase shiftermodulator. Optics Letters, 26(4):187-189, 2001. 



\title{
FMCW paper
}

This appendix consists of a published paper describing an optical distance ranging technique which was originally intended for use in the aOCT system. It was ultimately not used because tunable lasers of the required specifications were not commercially available and there were not sufficient resources to develop one in-house. As such tunable sources become available this will likely replace time-domain optical coherence tomography. Note that the name "FMCW interferometry" is used here, however, in recent years (since the time this was published) "swept-source optical coherence tomography" (SS-OCT) has been commonly adopted as the name for this technique.

\section{Distance ranging to biological tissue using fibre-optic Fabry-Perot, short tuning range $\mathrm{FMCW}$ interferometry}

Julian J. Armstrong, David D. Sampson

Optical+Biomedical Engineering Laboratory, Department of Electrical and Electronic Engineering, University of Western Australia, Nedlands, WA 6907, Australia Tel.: +61-8-9380-3916 Fax.: +61-8-9380-1065

Email: julian-a@ee.uwa.edu.au

\begin{abstract}
We present a low-complexity optical ranging system intended for distance ranging to moving biological tissue. The optical ranging technique used is short tuning range, optical FMCW interferometry, in a Fabry-Perot configuration, with a common-path downlead. We demonstrate ranging to a bovine muscle tissue sample over a $250 \mathrm{~mm}$ range with an average resolution of $0.55 \mathrm{~mm}$.

\section{A.1 Introduction}

There are few light-based systems sufficiently sensitive to detect the weak reflections typically present in biological and biomedical imaging and distance measurement applications. Optical coherence tomography (OCT) has been shown to be very effective for high-resolution measurements of reflectivity profiles. However, to date the geometrical and physical limitations of all OCT systems demonstrated have meant that for high-speed scans the technique can by used only over a limited distance range. For an OCT system capable of motion-artefact-free in
\end{abstract}


vivo imaging, resolutions of $10 \mu \mathrm{m}$ have been reported [1], but to date the largest distance range reported for an OCT system is 2.3 centimetres [2], and ranges of a few millimetres are typical [1]. There exist biological applications for distance ranging over $0-250 \mathrm{~mm}$ dimensions; these include investigation of hollow tube organs in the human body, such as the upper airway, ear-nose-throat passages, the urethra and the bladder. External profiling of the human body may also be a possible application. An alternative light-based reflectometry technique that may be better suited to these applications is frequency modulated continuous wave (FMCW) interferometry. The use of FMCW interferometry in systems designed for subsurface imaging of stationary biological material has been demonstrated in a variety of configurations, including implementations via bulk-optic [3], [4], and fibre-optic Michelson interferometers [5]. One of the key issues in FMCW interferometry is that the resolution of the position measurements is inversely related to the total optical frequency excursion. For example, for a source capable of providing an optical frequency sweep of $\Delta F=300 \mathrm{GHz}$, the smallest resolvable distance between any two reflectors in air, approximately given by $c / 2 \Delta F$, would be $1 \mathrm{~mm}$. Whilst this limit holds when the axial distribution of multiple reflectors is to be determined, the same resolution can be achieved using a much smaller optical frequency excursion, if all that is to be determined is the location of a dominant reflection due to an air-tissue interface.

We present an optical FMCW distance ranging system that can range to biological tissue over a range of greater than $250 \mathrm{~mm}$. The ranging system is relatively simple, and employs a small number of optical and electronic components. The optical source is a standard $1300 \mathrm{~nm}$ communications-grade laser that produces a very small optical frequency tuning range of $10 \mathrm{GHz}$. The interferometer is a Fabry-Perot configuration in which the target comprises one reflector. The resolution of the ranging measurement depends on signal strength, but for signal return losses of $-50 \mathrm{~dB}$, we have demonstrated resolutions of $0.17 \mathrm{~mm}$. When distance ranging to a sample of bovine muscular tissue which had signal return losses varying from -60 to $-75 \mathrm{~dB}$, we measured an average resolution of approximately $0.55 \mathrm{~mm}$ over the $250 \mathrm{~mm}$ range.

An important advantage of this system is the fact that good distance resolution can be achieved using a small frequency tuning range. The use of the Fabry-Perot configuration, which we believe is novel for this type of application, ensures that, in contrast to OCT, the downlead to the probe is common path for the interferometer. This is beneficial in situations where external influences such 
as vibration, stress and temperature variations are present.

\section{A.2 Principle}

The general principle of FMCW interferometry, also known as optical frequencydomain reflectometry (OFDR), is well known [6], so a simplified description will suffice here. We consider a Fabry-Perot-type interferometer which has two reflectors referred to as the reference and sample reflectors. Their reflectivities are small enough that multiple reflections need not be considered. A narrow-linewidth source at the interferometer input has its optical frequency modulated by a symmetric triangle waveform with repetition rate $f_{m}$. Signals returning from the two reflectors to recombine at the output will have different delay times through the interferometer, and so will have different optical frequencies due to the timedependent optical frequency modulation at the input. Therefore, a beating in intensity will be observed at the interferometer output. For a optical path length difference (in air) of $d$, the beat frequency is given by

$$
f_{b}=\frac{4 f_{m} \Delta F d}{c}
$$

where $\Delta F$ is total optical frequency excursion and $\mathrm{c}$ is the speed of light.

From Equation A.1, the beat frequency, $f_{b}$, is proportional to the path length difference. Given that we know the position of the reference reflector, then the position of the sample reflector may be determined. However, the situation is modified when one of the reflectors is moving, as might easily occur when the sample reflector is an in vivo biological sample. If its velocity component in the direction of the probe is $v$, then the frequency of the light reflected from it will undergo a Doppler shift of $f_{d}=2 v / \lambda$, where $\lambda$ is the wavelength of the source. The use of the triangular waveform allows us to compensate for the Doppler shift. During the upward and downward sweeps of the source optical frequency, the beat frequency observed due to the optical interference will be augmented or diminished by exactly $f_{d}$, i.e.,

$$
f_{b, \text { up }}=f_{b}+f_{d}, \quad \text { and } \quad f_{b, \text { down }}=f_{b}-f_{d} .
$$

From these beat frequencies we can determine $f_{b}$, which gives us the path length difference $d$, and also $f_{d}$, which can be used to determine the velocity of the sample. 


\section{A.3 Experimental system}

The configuration of the distance ranging system is shown in Figure A.1. The laser source used was a fibre-pigtailed $1300 \mathrm{~nm}$ distributed feedback laser (Fujitsu FLD130F2KD), followed by a $-65 \mathrm{~dB}$ isolator used to prevent back reflections from affecting the laser's stability. The bias current of the laser was modulated with a $5 \mathrm{kHz}$ triangle wave of sufficient amplitude to achieve an optical tuning range of $10 \mathrm{GHz}$. The average power of the source was $1.75 \mathrm{~mW}$. A $2-150 \%$ fibre coupler was used to direct light to the probe optics. At the probe distal end a 0.23 pitch anti-reflection coated GRIN lens was used both to weakly focus the light to the sample, and to collect the backscattered signal and couple it back into the fibre. The lens was separated from the cleaved end of the fibre by a small air gap, so that there was a $4 \%$ reflection at the fibre end. This formed the reference reflection, and the sample placed in the path of the focussed beam provided the second reflector. For initial testing the sample used was a mirror, but this was later replaced with a thick section of bovine muscle tissue. The light returning from the probe was directed by the coupler to the photodetector, comprising an avalanche photodiode followed by a transimpedance amplifier. A data acquisition card digitised the resulting signal, and the signal processing was performed in software.

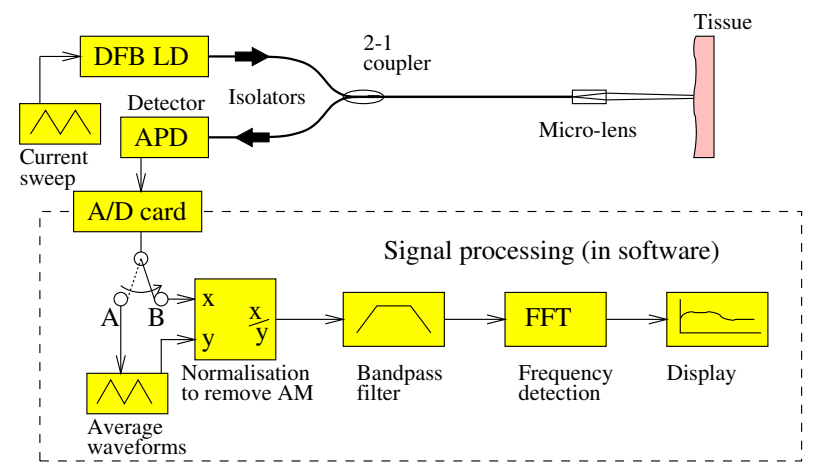

Figure A.1: A schematic of the distance ranging system.

As the modulation of the laser current causes both a frequency modulation and an intensity modulation, the intensity modulation must be removed to obtain a normalised waveform so that the beat frequency can be determined. To do this, a "reference" waveform can be captured when there is no sample present. Then, when a sample is introduced, the waveform containing the beat frequencies and the intensity modulation is divided by the reference waveform. This is illustrated in Figure A.2, where Figure A.2(a) shows a measured raw waveform, and Figure 
A.2(b) shows the subsequent normalised waveform.

To extract the distance and velocity information from the normalised waveform, the beat frequency is calculated in both the up and down sweep sections using an interpolated discrete Fourier transform peak-finding method. The mean of the two beat frequencies is used to calculate the sample position, and the difference between the frequencies used for the sample velocity. The distance ranging measurements can be performed at a repetition rate of $f_{m}$, the source modulation frequency; $5 \mathrm{kHz}$ was used in this case, which is adequate for most biomedical applications. A limiting factor on the repetition rate is that the optical frequency shift is reduced at higher modulation frequencies [7].

\section{A.4 Results}
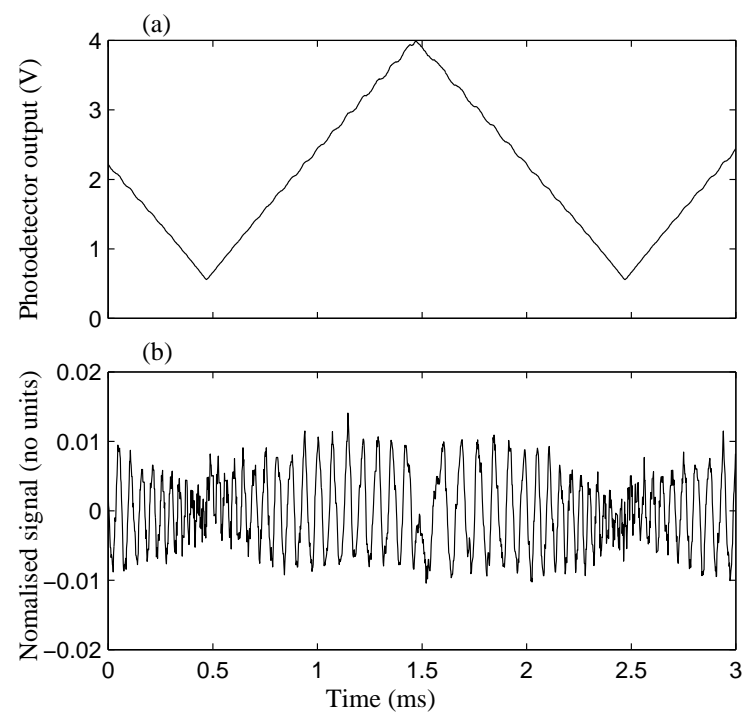

Figure A.2: Example of (a) an unprocessed photocurrent waveform, and (b) the corresponding normalised waveform where the intensity modulation has been removed.

An example of the results obtained with the system as described is shown in Figure A.3. A section of bovine muscle tissue in a rail-mounted sample holder was placed in front of the probe, and was moved by hand to five different positions along the rail, remaining stationary at each of the positions for approximately twenty seconds. The average power in the light focussed on the sample was $0.87 \mathrm{~mW}$. The signal strength fluctuated considerably due to the inhomogeneous nature of the tissue surface, however, the sample position was tracked well. Missing points on the plot are instances where the fluctuating signal strength 


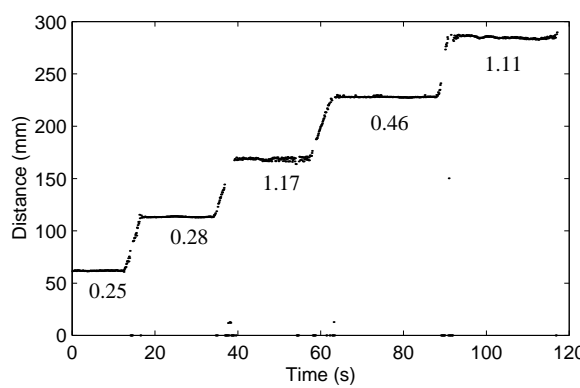

Figure A.3: Distance ranging to a sample of bovine muscle tissue. The numbers on the plot indicate the ranging resolution in millimetres for the periods when the sample was stationary.

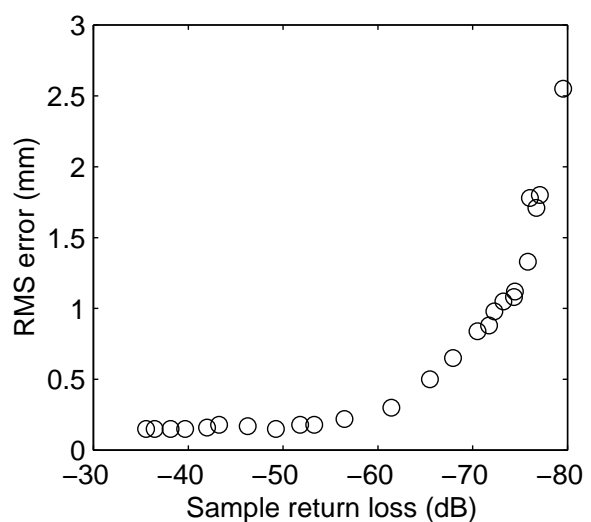

Figure A.4: Position resolution versus sample return loss.

dropped below the noise floor, or the sample velocity caused a Doppler shift which was greater than the system could compensate for, and thus no distance could be measured. The sample return loss, i.e., the ratio between light directed towards the sample and the backscattered light coupled into the probe, was in the range of -60 to $-75 \mathrm{~dB}$. The root-mean-square (RMS) variation in each of the measured positions when the sample was stationary are indicated on the figure. Note that with conventional FMCW signal processing, not taking into account Doppler shifts, the moving bovine tissue sample could not have been tracked while in motion.

In order to estimate the effect of variation in reflectivity on resolution in the distance ranging, a mirror was used as the sample and oriented in such a way as to allow only a small amount of light to re-enter the probe. By adjusting the mirror tilt, the amount of light re-entering the probe could be finely controlled. When a sample is stationary, the RMS variation in sample position is a good indication of distance ranging resolution. Figure A.4 shows a plot of RMS variation versus sample return loss. Each of these two parameters is affected by random noise in the system, and so each data point was measured using a ten second average. As the figure shows, there is a degradation in resolution as the signal to noise ratio is reduced by lower sample return loss. 


\section{A.5 Discussion and conclusions}

A problem with the Fabry-Perot configuration of the system described here is that as the reference reflection for the interferometer is near the end of the probe, the beat frequency approaches zero as the sample and reference separation approaches zero. Below a certain distance, the path length difference will not be large enough to generate a sufficiently large beat frequency to be measured accurately in the time period of the source modulation. If ranging close to the probe head is required, there are several possible approaches to reduce the minimum distance. These include increasing the optical frequency tuning range of the source, or using a reference reflection which is placed further back in the fibre (typically a few tens of millimetres). Another limitation in the system is that there is a maximum velocity of the sample beyond which the Doppler shift will be great enough to make the beat frequency either too high or too low to be measurable. For example, using a tuning range of $10 \mathrm{GHz}$ with a repetition rate of $5 \mathrm{kHz}$, ranging to a sample at $100 \mathrm{~mm}$, will mean a maximum velocity of $43 \mathrm{~mm} / \mathrm{s}$ can be detected. However, these limitations aside, the technique does allow distance measurement to weakly reflecting surfaces over a useful range of distances and does so with a system very low in complexity, and without requiring sensitive moving components or specially matched reference fibre lengths.

In conclusion, a low-complexity FMCW reflectometry distance ranging system, using a very low optical frequency tuning range communications-grade laser, with a downlead-insensitive probe, has been presented. The system has been demonstrated to be capable of ranging to a bovine muscle tissue sample over a range of $250 \mathrm{~mm}$ with almost half millimetre average resolution, and capable of tracking the sample during motion. This type of system could form the basis of biomedical applications for monitoring and quantitative structural determination.

\section{References}

[1] G. J. Tearney, B. E. Bouma, and J. G. Fujimoto, “High-speed phase- and groupdelay scanning with a grating-based phase control delay line," Opt. Lett., vol. 22, pp. 1811-1813, Dec. 1997.

[2] K. K. M. B. D. Silva, A. V. Zvyagin, and D. D. Sampson, "Extended range, rapid scanning optical delay line for biomedical interferometric imaging," Electron. Lett., vol. 35, no. 17, pp. 1404-1406, 1999. 
[3] U. Haberland and H. J. Schmitt, "Optical coherent imaging of volume scattering media with near infrared tunable laser," in 18th Annual International Conference of the IEEE Engineering in Medicine and Biology Society, Imaging Systems Analysis and Modelling, (Amsterdam), pp. 834-835, IEEE, 1996.

[4] F. Lexer, C. K. Hitzenberger, A. F. Fercher, and M. Kulhavy, "Wavelengthtuning interferometry of intraocular distances," Appl. Opt., vol. 36, pp. 65486553, Sept. 1997.

[5] S. R. Chinn, E. A. Swanson, and J. G. Fujimoto, "Optical coherence tomography using a frequency-tunable optical source," Opt. Lett., vol. 22, pp. 340-342, Mar. 1997.

[6] R. Passy, N. Gisin, J. P. von der Weid, and H. H. Gilgen, "Experimental and theoretical investigations of coherent OFDR with semiconductor laser sources," J. Lightwave Technol., vol. 12, pp. 1622-1630, Sept. 1994.

[7] G. Jacobsen, H. Oleson, F. Birkedahl, and B. Tromborg, “Cuurent/frequencymodulation characteristics for directly optical frequency-modulated injection lasers at $830 \mathrm{~nm}$ and $1300 \mathrm{~nm}, "$ Electron. Lett., vol. 18, pp. 874-876, Sept. 1982. 


\section{Publication list}

This is a list of the publications that I co-authored during the period of this $\mathrm{PhD}$ project. PDFs and online multimedia material of some of these publications are available on the website of the Optical+Biomedical Engineering Lab (OBEL):

http: //obel.ee.uwa.edu.au/publications/

[I] A. V. Zvyagin, K. K. M. B. D. Silva, S. C. Moore, J. J. Armstrong, D. D. Sampson, "Optical delay line with centimetre range and metre-per-second scan rate for motion-artifact-free imaging by fibre-optic coherence tomography", Postdeadline paper no. 1, Australian Conference on Optical Fibre Technology (ACOFT-24), Sydney, New South Wales, July 4-7, 1999.

[II] J. J. Armstrong, D. D. Sampson, “Distance ranging to biological tissue using fibre-optic Fabry- Perot, short tuning range FMCW interferometry" in 14th International Conference on Optical Fiber Sensors, Florence, Italy, A. G. Mignani, H. C. Lefevre, Editors, Proc. of SPIE, vol. 4185, pp. 366-369, 2000.

[III] J. J. Armstrong, D. D. Sampson, “Distance ranging to biological tissue using fibre-optic Fabry-Perot, short tuning range FMCW interferometry," in Conference Abstracts and Poster Summaries, 13th Conference of the Australian Optical Society, paper MT14, Adelaide, December 10-15, 2000.

[IV] D. D. Sampson, B. A. Patterson, J. J. Armstrong, "Some biomedical applications of optical fibre sensors," in Proc. of Optoelectronics and Communications Conference/Integrated Optics and Optical Fibre Communications Conference, pp. 482-485, 2001 (invited).

[V] Y. Awaji, J. J. Armstrong, W. Chujo, and D. D. Sampson, "The effects of input polarization and detuning on Differential-Phase-to-Intensity Conversion (DPIC) waveform", in Photonics Switching Workshop, Japan, 2001.

[VI] Y. Awaji, J. J. Armstrong, W. Chujo, and D. D. Sampson, "DPIC waveform dependency on polarization and detuning," in Microwave and Millimetre Wave Photonics Workshop, Japan, 2001.

[VII] D. D. Sampson, A. V. Zvyagin, E. D. J. Smith, K. K. M. B. Dilusha Silva, J. J. Armstrong, "Optical coherence tomography: technology and applications," 
in Australian Conference on Optical Fibre Technology (ACOFT-27), Sydney, NSW, July 7-11, 2002 (invited).

[VIII] D. D. Sampson, A. V. Zvyagin, J. J. Armstrong, "Some applications of optical coherence tomography," in Focus on Microscopy, Technical Digest, pp. 27, Kaohsiung, Taiwan, April 2002 (invited).

[IX] J. J. Armstrong, M. S. Leigh, I. D. Walton, A. V. Zvyagin, S. A. Alexandrov, S. Schwer, D. D. Sampson, D. R. Hillman, P. R. Eastwood, "In vivo size and shape measurement of large hollow organs using endoscopic long-range optical coherence tomography", Optics Express, vol. 11, no. 15, pp. 18171826, 2003.

online journal link: http: //www. opticsexpress.org/abstract.cfm? URI $=O P E X-11-15-1817$

[X] J. J. Armstrong, M. S. Leigh, D. R. Hillman, P. R. Eastwood, D. D. Sampson, "In vivo size and shape measurement of the human upper airway using an endoscopic long-range OCT system," presented at Advances in Optics for Biotechnology, Medicine and Surgery, Poster 4, Banff, Alberta, Canada, August 2003.

[XI] P. R. Eastwood, J. J. Armstrong, A.V. Zvyagin, I. D. Walton, S. A. Alexandrov, S. Schwer, M. S. Leigh, D. R. Hillman, D. D. Sampson, "In vivo size and shape measurement of the human upper airway using endoscopic longrange optical coherence tomography (OCT)," in World Congress on Medical Physics and Biomedical Engineering, 24-29 August, Sydney, Australia, CD-ROM Digest, 2003.

[XII] J. J. Armstrong, M. S. Leigh, I. D. Walton, S. Schwer, A. V. Zvyagin, S. A. Alexandrov, D. D. Sampson, D. R. Hillman, P. R. Eastwood, “An endoscopic long-range optical coherence tomography system and its application to in vivo size and shape measurement of large hollow organs," in 16th International Conference on Optical Fiber Sensors, Nara, Japan, K. Hotate, H. Nagai, Editors, pp. 722-725, October 2003.

[XIII] P. R. Eastwood, J. J. Armstrong, A. V. Zvyagin, I. Walton, S. A. Alexandrov, S. Schwer, M. S. Leigh, D. D. Sampson, D. R. Hillman, "In vivo size and shape measurement of upper airway using endoscopic optical coherence 
tomography (OCT)," in Australasian Sleep Association Annual Scientific Meeting, Auckland, New Zealand, October 2003.

[XIV] D. D. Sampson, A. V. Zvyagin, E. D. J. Smith, I. D. Walton, J. J. Armstrong, K. K. M. B. D. Silva, "The frequency-domain delay line in optical coherence tomography: design, capabilities, and limitations," in Conference on Lasers and Electrooptics-Pacific Rim (CLEO-PACRIM), Taiwan, paper W2E-(12)-1, July 2003 (invited).

[XV] A. V. Zvyagin, K. K. M. B. D. Silva, S. A. Alexandrov, T. R. Hillman, J. J. Armstrong, T. Tsuzuki, and D. D. Sampson, "Refractive index tomography of turbid media by bifocal optical coherence refractometry," Optics Express, vol. 11, pp. 3503-3517, 2003.

[XVI] P. R. Eastwood, J. J. Armstrong, A. V. Zvyagin, I. Walton, S. A. Alexandrov, S. Schwer, M. S. Leigh, D. D. Sampson, D. R. Hillman, “In vivo size and shape measurement of the upper airway using endoscopic optical coherence tomography (OCT)," in 14th European Respiratory Society Annual Congress, European Respiratory Journal, vol. 24 (Supplement 48): P2743, September 2004.

[XVII] A.V. Zvyagin, S.A. Alexandrov, K.K.M.B.D. Silva, T.R. Hillman, J. J. Armstrong, T. Tsuzuki, D.D. Sampson, "Refractive index tomography of turbid media by bifocal optical coherence refractometry," in Coherence Domain Optical Methods and Optical Coherence Tomography in Biomedicine VIII, edited by Valery V. Tuchin, Joseph A. Izatt, James G. Fujimoto, Bellingham, WA, USA, Proceedings of SPIE Vol. 5316, pp. 155-166, 2004.

[XVIII] D. D. Sampson, J. J. Armstrong, M. S. Leigh, D. R. Hillman, P. R. Eastwood, "Imaging human upper airway architecture dynamically using optical coherence tomography," in 8th International Conference on Optics Within Life Sciences, Melbourne, Australia, pp. 143-144, November 28-December 1, 2004 (invited).

[XIX] D. D. Sampson, T. R. Hillman, J. J. Armstrong, "Optical coherence tomography" in Australian Optical Society News, vol. 19, no. 2, pp. 30-35, June 2005 (invited).

[XX] P. R. Eastwood, J. J. Armstrong, M. S. Leigh, A. Paduch, D. D. Sampson, J. H. Walsh, D. R. Hillman, "Assessing upper airway structure and function 
with anatomic optical coherence tomography (aOCT)," in Annual Scientific Meeting of the Australasian Sleep Association, Gold Coast, QLD, Australia, 7-9 October, 2005 (invited).

[XXI] M. S. Leigh, J. J. Armstrong, A. Paduch, D. D. Sampson, J. H. Walsh, D. R. Hillman, P. R. Eastwood, "Quantitative imaging of the human upper airway: instrument design and clinical studies" in Saratov Fall Meeting: Optical Technologies in Biophysics \& Medicine VII, (V. V. Tuchin, ed.), Saratov, Russia, September 2005 (invited).

[XXII] D. D. Sampson, S. G. Adie, S. A. Alexandrov, J. J. Armstrong, T. R. Hillman, M. S. Leigh, "Optical coherence tomography: from fundamentals to clinical medical imaging," in Australian Conference on Lasers and Spectroscopy, Auckland, New Zealand, Paper TuE1, 5-9 December 2005 (invited).

[XXIII] J. J. Armstrong, M. S. Leigh, D. D. Sampson, J. H. Walsh, D. R. Hillman, and P. R. Eastwood, "Quantitative Upper Airway Imaging with Anatomic Optical Coherence Tomography," American Journal of Respiratory and Critical Care Medicine, vol. 173, pp. 226-233, 2006.

online journal link: http: / / a jrccm. ats journals.org/cgi/content / abstract/173/2/226

[XXIV] T. R. Hillman, S. G. Adie, V. Seemann, J. J. Armstrong, S. L. Jacques, and D. D. Sampson, "Correlation of static speckle with sample properties in optical coherence tomography," Optics Letters, vol. 31, pp. 190-192, 2006.

[XXV] D. D. Sampson, S. G. Adie, S. A. Alexandrov, J. J. Armstrong, T. R. Hillman, M. S. Leigh, "Tissue imaging with optical coherence tomography," presented at Biophotonics in Australia, Sydney, Australia, February, 2006 (invited).

[XXVI] M. S. Leigh, J. J. Armstrong, A. Paduch, J. H. Walsh, D. R. Hillman, P. R. Eastwood, D. D. Sampson, "Anatomical Optical Coherence Tomography: Instrument design and application to the upper airway," IEEE Transactions on Medical Imaging (submitted).

[XXVII] J. J. Armstrong, M. S. Leigh, D. R. Hillman, P. R. Eastwood, and D. D. Sampson, "Anatomical Optical Coherence Tomography of the Human Upper Airway," in "Optical Coherence Tomography: Technology and Appli- 
cations," editors Wolfgang Drexler and James G. Fujimoto, to be published by Springer (invited, in press).

[XXVIII] M. S. Leigh, J. J. Armstrong, A. Paduch, D. R. Hillman, P. R. Eastwood, D. D. Sampson, "Quantitative upper airway imaging using anatomical optical coherence tomography," Focus on Microscopy, Perth, Western Australia, pp. 132, 9-12 April, 2006.

[XXIX] J. Wang, G. A. Tetlow, A. D. Lucey, J. J. Armstrong, M. S. Leigh, D. D. Sampson, J. H. Walsh, P. R. Eastwood, D. R. Hillman, "Dynamics of the human upper airway: On the development of a three-dimensional computational model," World Congress on Medical Physics and Biomedical Engineering 2006, "Imaging the Future Medicine", Seoul, Korea, 27 August - 1 September 2006 (submitted). 



\section{FDODL theory}

This appendix is a short introduction to the theory behind the operation of the FDODL. It is not an intuitive explanation of source of the variable optical delay in the FDODL; this is given in Section 3.2.1 of this thesis. It is also not a full explanation of the theory, as this has been well covered previously - firstly, the initial reports of the delay line as used in optical coherence tomography $[87,88]$, and in further publications presenting more complete analyses: [143] provided a treatment with a full derivation of the phase function and optical simulation verification, [144] covered the theory focussing on dispersion properties as a function of the grating tilt, [89] covered grating tilt and translation with a less complete dispersion treatment, followed by [129] which presents a complete treatment of delay and dispersion properties. The mode of operation of the FDODL as an achromatic phase shifter has also been described [145].

The schematic diagram of the FDODL for this explanation is given in Figure C.1.

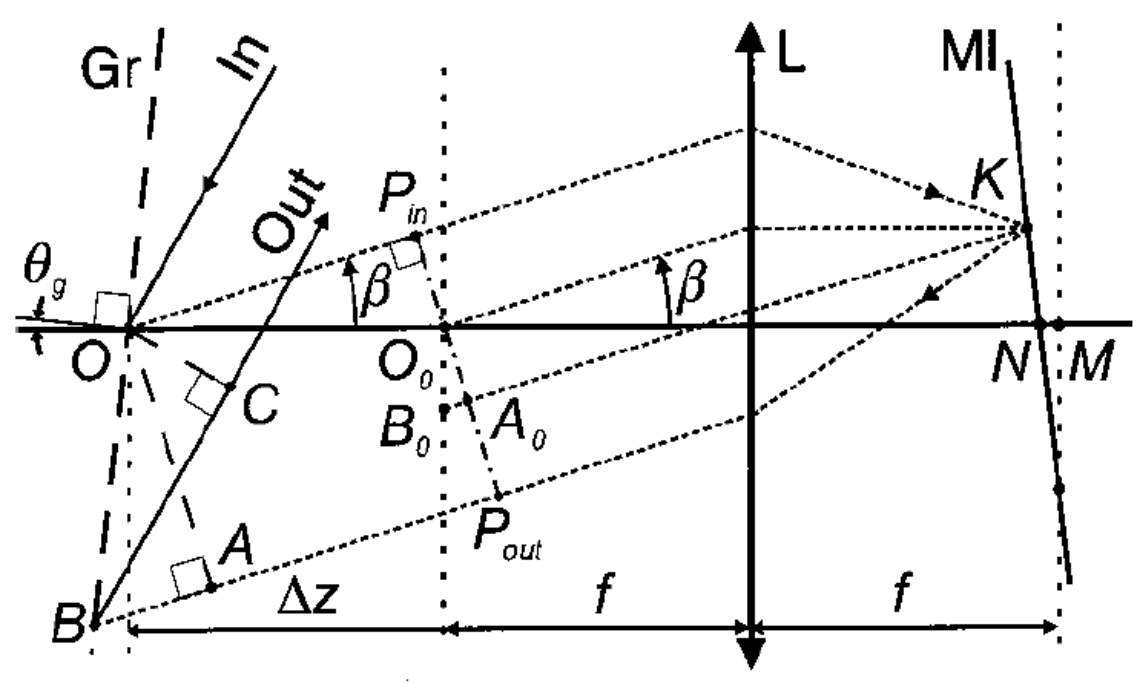

Figure C.1: Detailed schematic diagram of the FDODL including galvanometer rotation and translation, and grating tilt and translation. GR, grating; MI, mirror; f, lens focal length. Reproduced from [89].

To derive the group and phase delay properties of the FDODL as used in the aOCT system, we use the expression for the total optical phase shift through the FDODL [89],

$$
\Delta \psi=2 k x_{0} \theta+\frac{4 \pi m \theta f\left(k-k_{0}\right)}{p k_{0}}
$$


where $k=2 \pi / \lambda$ is the wavenumber, $\theta$ is the tilt angle of the galvanometer mirror, $\mathrm{m}$ is the diffraction order, $f$ is the focal length of the lens, and $p$ is the pitch of the grating. $k_{0}$ is the wavenumber of the central wavelength, which is aligned so that it passes though the centre of the lens.

Note that the phase shift expression only contains the phase shift which is dependent on the galvanometer tilt and the differential phase shift for the different wavelengths.

Including the static phase delay in the interferometer we then have

$$
\psi=2 k \delta+2 k x_{0} \theta+\frac{4 \pi m \theta f\left(k-k_{0}\right)}{p k_{0}}
$$

where $\delta$ is the total phase mismatch in the optical path in the interferometer. The phase and group delay in the interferometer can then be expressed as

$$
\begin{aligned}
t_{p} & =\frac{1}{c} \frac{\psi\left(k_{0}\right)}{k_{0}}=\frac{2 \delta}{c}+\frac{2 \theta x_{0}}{c} \\
t_{g}=\frac{1}{c} \frac{d \psi}{d k}\left(k_{0}\right) & =\frac{2 \delta}{c}+\frac{2 \theta x_{0}}{c}+\frac{4 \pi m \theta f}{c p k_{0}}
\end{aligned}
$$

When the phase and group delays are equal, this means that the interference fringes and the envelope of the fringes move together as the reference arm delay is scanned. This is the case when a translating mirror is used as a delay line rather than an FDODL. To derive this, consider the phase shift due to a translating mirror, $\Delta \psi=2 k z$ where $z$ is the mirror position. Then, including the static phase $\delta$ we get

$$
\begin{aligned}
t_{t m, p} & =\frac{1}{c} \frac{\psi\left(k_{0}\right)}{k_{0}}=\frac{2 \delta}{c}+\frac{z}{c} \\
t_{t m, g} & =\frac{1}{c} \frac{d \psi}{d k}\left(k_{0}\right)=\frac{2 \delta}{c}+\frac{z}{c}
\end{aligned}
$$

so $t_{t m, p}=t_{t m, g}$ as expected. For the FDODL case, examination of the expressions for $t_{p}$ and $t_{g}$ shows that this occurs for the limiting case of a galvanometer offset which is large so that the second term in the expression for $t_{g}$ becomes negligible. Note, however, that a larger offset also requires a larger mirror which means a greater mass and therefore a limit on the speed of operation of the galvanometer.

The smallest galvanometer mirror size is possible when $x=0$. For this case, the phase delay $t_{p}$ is constant with respect to the galvanometer angle $\theta$. Then, there is no phase modulation of the interference signal, and an envelope which is 
fringe free is detected. The envelope amplitude is a function of the interferometer static phase and this causes signal fading as the amplitude can be zero for certain values of the phase. To avoid the phase fading and also to move the detection bandwidth to avoid baseband noise, a phase modulator is used in the system to re-introduce fringes under the envelope, as mentioned in Chapter 3.

For the more general case of non-zero $\Delta z$ and $\theta_{g}$, the phase function $\Delta \psi(k)$ up to a second order Taylor expansion about $k_{0}$ is given by [75]

$\Delta \psi=2 x_{0} \theta k_{0}+\left(2 x_{0} \theta+\frac{4 \pi m f \theta}{p k_{0} \cos \theta_{g}}\right)\left(k-k_{0}\right)-\left(\frac{8 p i^{2} m^{2} \Delta z}{p^{2} k_{0}^{3} \cos ^{2} \theta_{g}}+\frac{8 p i^{2} m^{2} f \theta \sin \theta_{g}}{p^{2} k_{0}^{3} \cos ^{2} \theta_{g}}\right) \frac{\left(k-k_{0}\right)^{2}}{2 !}$

where $\Delta z$ is the grating offset and $\theta_{g}$ is the grating tilt.

The group delay dispersion is calculated from the phase function using

$$
\begin{aligned}
G D D & =\left.\frac{1}{c^{2}} \frac{\partial^{2} \psi(k)}{\partial k^{2}}\right|_{k=k_{0}} \\
& =-\left(\frac{8 p i^{2} m^{2} \Delta z}{p^{2} c^{2} k_{0}^{3} \cos ^{2} \theta_{g}}+\frac{8 p i^{2} m^{2} f \theta \sin \theta_{g}}{p^{2} c^{2} k_{0}^{3} \cos ^{3} \theta_{g}}\right)
\end{aligned}
$$

and this expression shows that the static dispersion of the reference arm can be controlled be (and is linearly proportional to) the grating offset. This is utilized in the $a \mathrm{OCT}$ system to counteract the dispersion mismatch caused by the difference in optical fibre and air paths in the sample and reference arms. Additionally, the tilt $\theta_{g}$ can be used to controlled the dynamic dispersion of the FDODL, however this is not required for the $a \mathrm{OCT}$ system and therefore $\theta_{g}$ is set to zero.

Figure C.2 is a photograph of the FDODL as it was implemented. High quality optical mounting hardware was necessary because of the the large path-lengths involved and high precision and stability required to achieve correct alignment. 


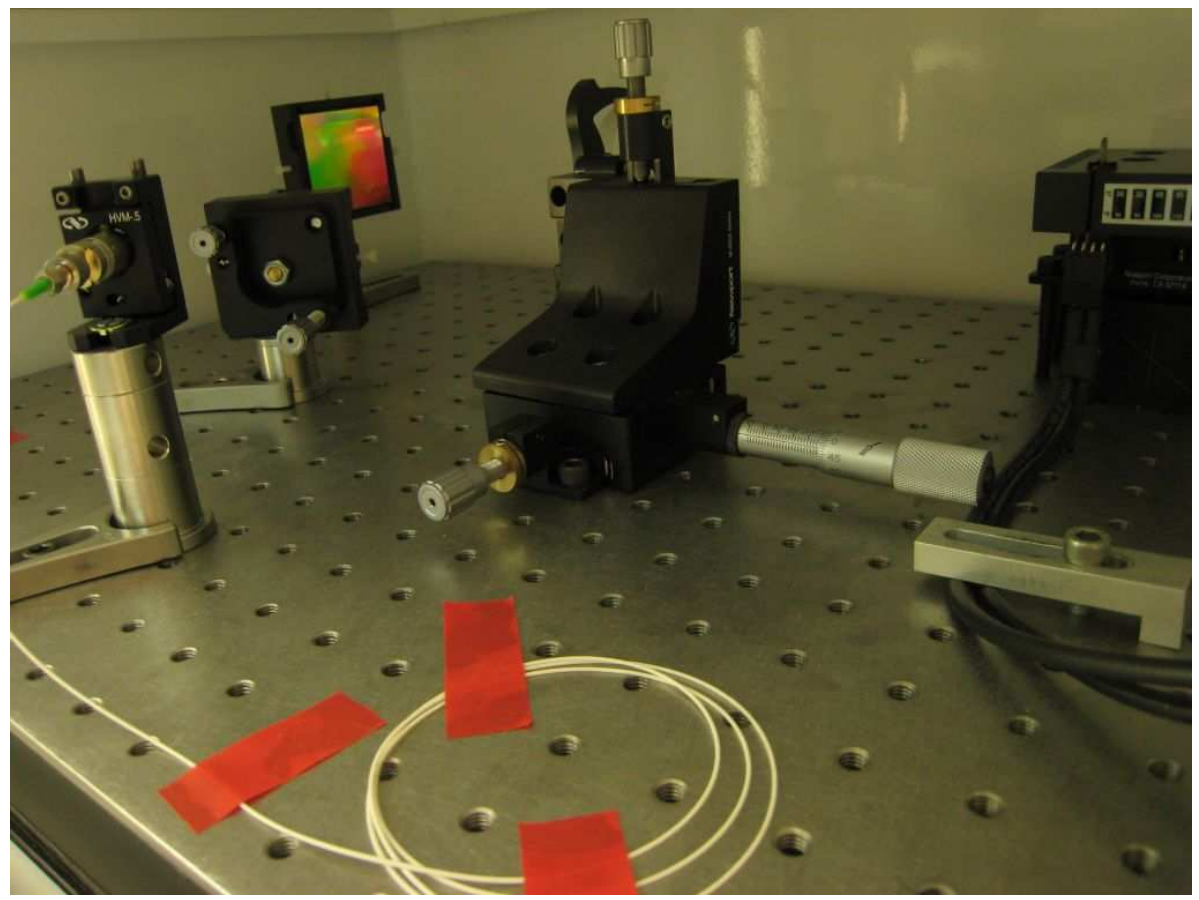

Figure C.2: The FDODL as implemented. It is mounted on an optical breadboard and enclosed in a tight-fitting metal box to reduce vibration and temperature fluctuations which would cause mis-alignment and phase drift. 


\section{Basic OCT signal generation theory}

This appendix is a basic introduction to the theory of signal generation in timedomain OCT systems. Detailed descriptions are beyond the scope of this thesis and have been covered well previously $[75,116]$.

We start by considering the simple Michelson interferometer shown in Figure D.1. The interferometer is illuminated by a light source (typically polychromatic), two arms are terminated by a reference reflector and a sample, and the optical intensity is detected at the output port. The light from the source is split into the reference field $E_{r i}$ and sample field $E_{s i}$. The sample field impinges on the sample through focussing and possibly scanning optics. The output contains the fields $E_{r}$ and $E_{s}$ which interfere, producing the output intensity

$$
I_{d}=\left\langle\left|E_{r}+E_{s}\right|^{2}\right\rangle=I_{r}+I_{s}+2 \operatorname{Re}\left\{\left\langle E_{r}^{*}(t+\tau) E_{s}(t)\right\rangle\right\}
$$

where $I_{r}$ and $I_{s}$ are the mean intensities from the reference and sample arms respectively. The time $\tau$ is the optical time delay between the reference and the sample arms which may be modulated. This shows that the interferometer output is a cross-correlation of the sample and reference beams, and interference may be seen in the detected signal depending on the degree of coherence between the two fields.

If we make the assumption that the reference and sample arms behave as ideal reflectors (of variable reflectivity but they do not alter the coherence or spectral properties of the fields), the cross-correlation signal is given by

$$
\operatorname{Re}\left\{\left\langle E_{r}^{*}(t+\tau) E_{s}(t)\right\rangle\right\}=|\Gamma(\tau)| \cos \left[2 \pi \tau c / \lambda_{0}+\phi(\tau)\right]
$$

where $\Gamma$ is the complex coherence function with phase $\phi, \lambda_{0}$ is the centre wavelength of the source and $c$ is the speed of light. The coherence function $\Gamma$ and the spectral density of the source are a Fourier transform pair, as given by the WienerKhinchin theorem. Note that we also assume that the light fields are scalar, stationary, ergodic and random. A convenient source spectral shape to use is the Gaussian function, as $\Gamma(\tau)$ is then also Gaussian, e.g.;

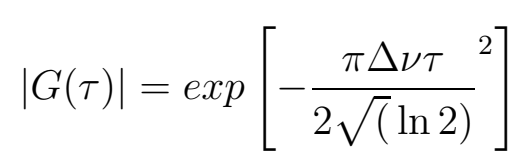




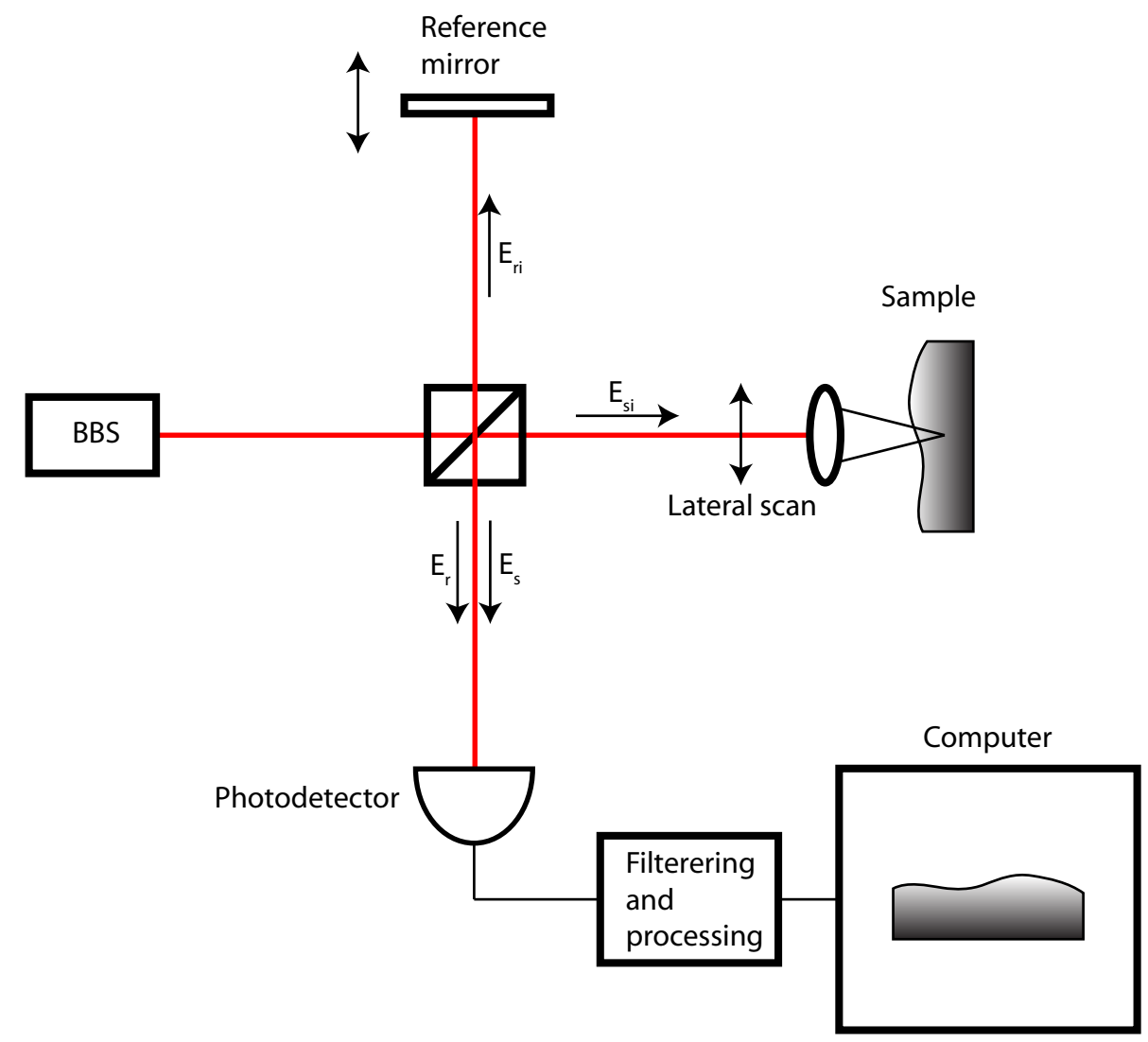

Figure D.1: Michelson low coherence interferometer. BBS, broadband source.

where $\Delta \nu$ is the width of the spectrum, then the interference signal (the a.c. part of $I_{d}$ ) is simply sinusiodal fringes modulated by a Gaussian function, where the centre of the Gaussian is located at $\tau=0$, i.e. zero path length mismatch between the reference and sample reflectors. The width of the Gaussian gives the coherence length (in the round-trip sense) $l_{c}$,

$$
l_{c}=\frac{2 \ln 2}{\pi} \frac{\lambda_{0}^{2}}{\Delta \lambda}
$$

For example, for a $1300 \mathrm{~nm}$ superluminescent diode with a Gaussian spectrum and spectral width of $100 \mathrm{~nm}$, the coherence length is $7.5 \mu \mathrm{m}$. This length defines the effective axial resolution of the reflectance profile resulting from the interference signal.

In order to detect the interferogram signal which contains the information about the reflectance profile of the sample arm, the relative time delay $\tau$ is modulated using a scanning delay in the reference arm. In the simplest implementation this is simply a mirror which is translated back and forth with a constant speed. 
An example simulated OCT signal, fringes modulated by a Gaussian envelope, is shown in Figure D.2(a). When there are multiple reflectors in the sample arm this results in the OCT signal as shown in Figure D.2(b). Note that the two right-most reflectors which are spaced too closely result in a distorted envelope from the coherent interaction between them; this is known as speckle. This is responsible for the typical "grainy" appearance of OCT images when compared to non-coherent imaging modalities such as brightfield microscopy.

(a)

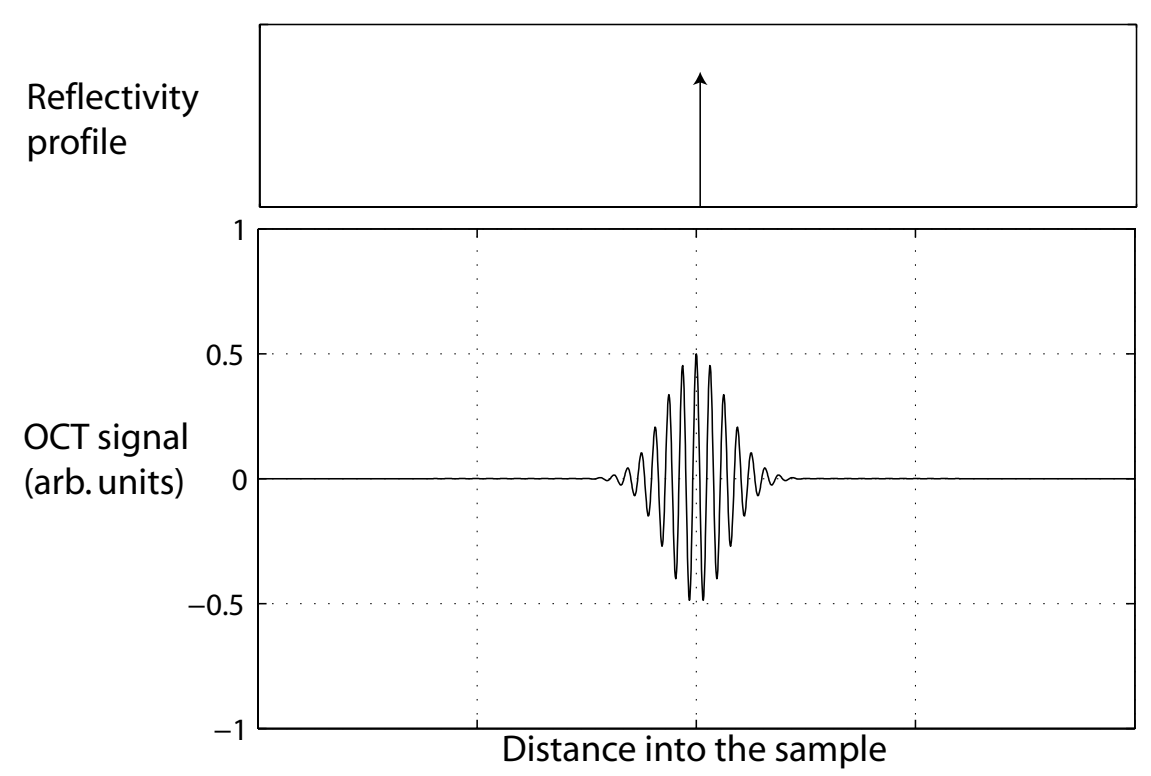

(b)

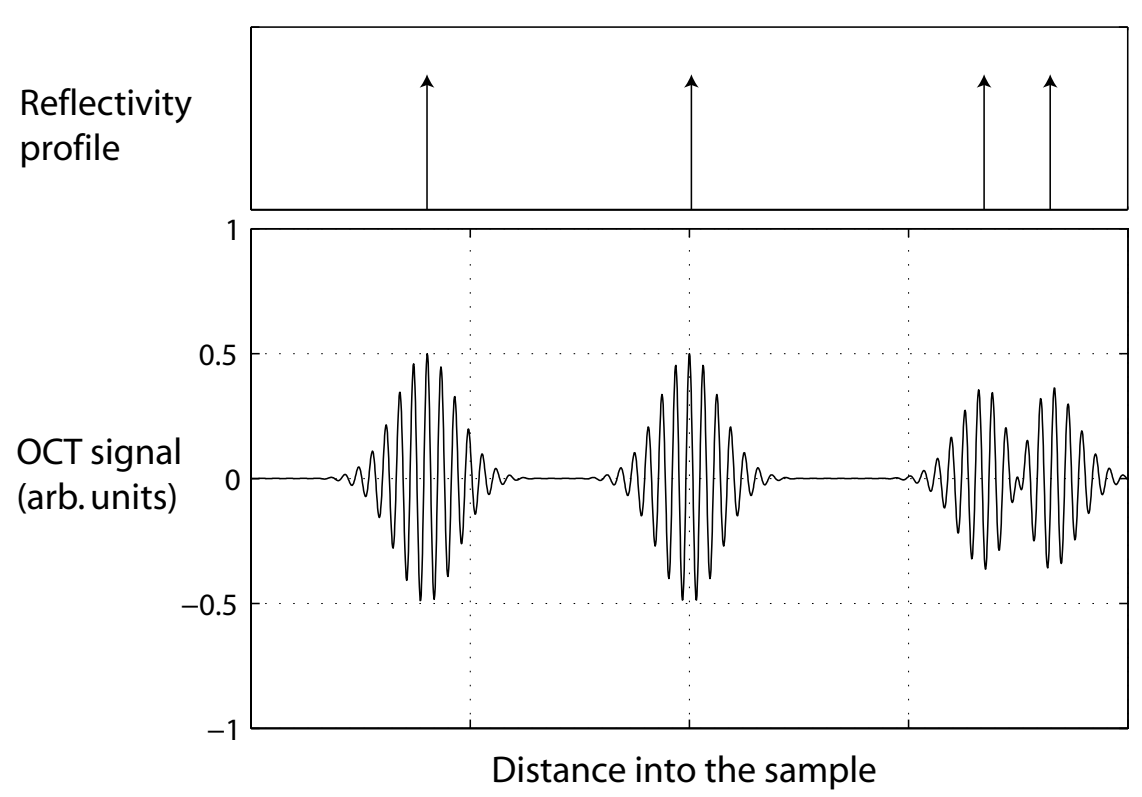

Figure D.2: Simulated examples of OCT signals for (a) a single reflector and (b) four reflectors. 